Department of Econometrics and Business Statistics

http://business.monash.edu/econometrics-and-business-

statistics/research/publications

\title{
High Dimensional Semiparametric Moment Restriction Models
}

Chaohua Dong, Jiti Gao and Oliver Linton

November 2017

Working Paper 17/17 


\title{
High Dimensional Semiparametric Moment Restriction Models
}

\author{
Chaohua Dong \\ Southwestern University of Finance and Economics, China \\ JiTI GAO \\ Monash University, Australia \\ OLIVER LINTON \\ University of Cambridge, UK
}

September 30, 2017

\begin{abstract}
Moment restriction semiparametric models, where both the dimension of parameter and the number of restrictions are divergent and an unknown function is involved, are studied using the generalized method of moments (GMM) and sieve method dealing with the nonparametric parameter. The consistency and normality for the GMM estimators are established. Meanwhile, a new test statistic is proposed for overidentification issue. Numerical examples are used to verify the established theory.
\end{abstract}

Key works: Generalized method of moments, high dimensional models, moment restriction, over-identification, sieve method

JEL classification: C12, C14, C22, C30

\section{Introduction and examples}

We consider a class of moment restriction models where there are many Euclidean valued parameters as well as unknown infinite dimensional functional parameters. The setting includes as a special case the partial linear regression model, Robinson [28], except in our case the number of covariates in the linear part may be large i.e., increase to infinity with sample size. 
For example, there are often many binary covariates whose effect can be restricted to be linear without a great loss of generality. Our model framework specifies which variables affect the outcome in a linear fashion and which variables affect the outcome nonlinearly. Nonparametric and "parametric" components can both be of interest in applications but present different statistical issues. Efficiency bounds and their achievability are quite different between the two cases. Inference procedures also differ substantially. In our framework, the model components are known beforehand and are clearly demarcated, and we can compare our results for the two different components with existing results in the relevant literatures. However, the parametric component itself is growing in complexity, which raises some new issues. We will use the Generalized Method of Moments (GMM) to deliver simultaneous estimation of all unknown quantities from a large dimensional moment vector. There is a considerable literature on GMM in parametric cases and recent work has mainly focussed on the extension to either many moment conditions Newey and Windmeijer [23], for example, or to the case where the number of Euclidean parameters is finite but there are unknown function-valued parameters (see, for example, Chen and Liao [8]; Chen et al. [9]). We will provide inference techniques for the parametric and nonparametric components of our model.

Suppose that

$$
\mathbb{E}\left[m\left(V_{i}, \alpha^{\top} X_{i}, g\left(Z_{i}\right)\right)\right]=0,
$$

for $i=1, \ldots, n$, where $m$ is a known vector of functions whose dimension $q$ is large, i.e., $q=$ $q(n) \rightarrow \infty$ as $n \rightarrow \infty$. Here, $\alpha$ is an unknown Euclidean valued parameter whose dimension $p=p(n) \rightarrow \infty$ as $n \rightarrow \infty$, while $g$ is an unknown smooth function. The observed vector variable $V_{i}$ typically represents a dependent variable and possible instrumental variables, while the observed vectors $X_{i}$ and $Z_{i}$ are explanatory variables, where $Z_{i}, V_{i}$ are of finite dimension, but the dimension of $X_{i}$ may diverge. We will consider the case where the parameter dimension $p$ grows to infinity but is smaller than $n$, similar to Portnoy [25], Portnoy [26] and Mammen [20]. This is the case in many applications. The moment restriction model (1.1) features high dimensionality in two folds: a high dimensional Euclidean parameter $(\alpha)$ that shows up in a single-index form, and a zero-mean function $m(\cdot)$ with divergent dimension that usually represents an error term. In addition, it includes an infinite dimensional unknown function $g(\cdot)$. Together this represents a new framework in the literature.

We suppose that a sample $\left(V_{i}, X_{i}^{\top}, Z_{i}^{\top}\right)_{i=1}^{n}$ is observed. We shall simultaneously estimate $\alpha$ and $g$ in parameter spaces defined below. As the function $g$ can be regarded as an element in some function space, which is infinite dimensional, all parameters are of high dimension. Moreover, we are also interested in transformations of $\alpha$ and functionals of $g$ for which we have plug-in estimators once we obtain the estimates of $\alpha$ and $g$. Chen et al. [9] study a fixed-dimensional moment restriction model containing an unknown function. The estimation 
strategy can be two step or profiled two-steps depending on the context. A similar approach is used again in Chen and Liao [8]. Kernel estimation techniques generally require an additional (albeit related) estimating equation and either two-step or profile methods are common, see, for example, [27].

To illustrate the proposal of model (1.1), we give the following examples.

Example 1.1 (Conditional moment restrictions): Let $W_{i}$ be a sub-vector of $\left(X_{i}^{\top}, Z_{i}^{\top}\right)^{\top}$ and $\rho\left(Y_{i}, \alpha^{\top} X_{i}, g\left(Z_{i}\right)\right)$ be a known $J$-dimensional vector of generalized residual function. Then, $(\alpha, g)$ is determined by a conditional moment restriction

$$
\mathbb{E}\left[\rho\left(Y_{i}, \alpha^{\top} X_{i}, g\left(Z_{i}\right)\right) \mid W_{i}\right]=0, \quad \text { almost surely }
$$

Let $\Phi_{k}(w)=\left(h_{1}(w), \ldots, h_{k}(w)\right)$ be a vector of functions that can approximate any square integrable function of $W$ in some sense arbitrarily as $k \rightarrow \infty$. Then, the conditional restriction implies

$$
\mathbb{E}\left[\rho\left(Y_{i}, \alpha^{\top} X_{i}, g\left(Z_{i}\right)\right) \otimes \Phi_{k}\left(W_{i}\right)\right]=0 .
$$

Denote $m\left(V_{i}, \alpha^{\top} X_{i}, g\left(Z_{i}\right)\right)=\rho\left(Y_{i}, \alpha^{\top} X_{i}, g\left(Z_{i}\right)\right) \otimes \Phi_{k}\left(W_{i}\right)$ where $V_{i}=\left(Y_{i}, W_{i}^{\top}\right)^{\top}$. Notice that the dimension of $m$ function is $J k$ which increases with $k$. Therefore, the pair $(\alpha, g)$ can be solved from the unconditional moment equation $\mathbb{E}\left[m\left(V_{i}, \alpha^{\top} X_{i}, g\left(Z_{i}\right)\right)\right]=0$. For example, suppose that $\Lambda\left(Z_{i}\right)=\alpha^{\top} X_{i}+\varepsilon_{i}$, where $\Lambda(\cdot)$ is an unknown monotone function and $\left(V_{i}, X_{i}\right)$ are observed. Under the conditional moment restriction $\mathbb{E}(\varepsilon \mid W)=0$ for some vector of instrumental variables $W$ we may obtain unconditional moment restrictions like (1.1) with $\Lambda$ being the unknown function of interest.

Example 1.2 (High dimensional partially linear endogenous model): Let $Y_{i}=\alpha^{\top} X_{i}+$ $g\left(Z_{i}\right)+e_{i}, i=1, \ldots, n$, where $\alpha \in \mathbb{R}^{p}$ and $e_{i}$ is an error term such that $\mathbb{E}\left[e_{i}\right]=0$ for all $i$. Here, $\left(X_{i}, Z_{i}\right)$ is endogenous in the sense that $\mathbb{E}\left[e_{i} \mid X_{i}, Z_{i}\right] \neq 0$. In the case where the dimensionality of $\alpha$ is fixed, there are various results available in the literature (see, for example, Robinson [28]; Gao and Liang [14]; Gao and Shi [15]; Härdle et al. [19]). To deal with the endogeneity, let $W_{i}$ be instrumental variable and define a set of valid instruments $\lambda_{i}=\lambda_{i}\left(W_{i}\right)$ with dimension $q$ and $q>p$.

Denote $m\left(V_{i}, \alpha^{\top} X_{i}, g\left(Z_{i}\right)\right)=\left(Y_{i}-\alpha^{\top} X_{i}-g\left(Z_{i}\right)\right) \lambda_{i}\left(W_{i}\right)$ with $V_{i}=\left(Y_{i}, W_{i}^{\top}\right)^{\top}$. Then, we have the moment condition $\mathbb{E}\left[m\left(Y_{i}, W_{i}, \alpha^{\top} X_{i}, g\left(Z_{i}\right)\right)\right]=0$, which can be used to identify the parameter $\alpha$ and nonparametric function $g(\cdot)$.

Example 1.3 (Discrete maximum likelihood): Suppose that $Y_{i}$ assumes either 0 or 1, and

$$
P\left(Y_{i}=1 \mid X_{i}, Z_{i}\right)=F\left(\alpha^{\top} X_{i}+g\left(Z_{i}\right)\right)
$$


for $i=1, \ldots, n$, where $\alpha, X_{i} \in \mathbb{R}^{p}$ and $Z_{i} \in \mathbb{R}$. The log likelihood function is

$$
\ln \prod_{i=1}^{n} F^{Y_{i}}\left(\alpha^{\top} X_{i}+g\left(Z_{i}\right)\right)\left[1-F\left(\alpha^{\top} X_{i}+g\left(Z_{i}\right)\right)\right]^{1-Y_{i}} .
$$

A sieve method can be used to estimate the unknown $g(\cdot)$, along with the estimate of $\alpha$. Suppose that the function $g(\cdot)$ can be approximated arbitrarily in some sense by a linear combination of $k$ known functions wrapped into a column vector $\Phi_{k}(\cdot)$, i.e. $g(z)-\beta^{\top} \Phi_{k}(z)$ is approaching zero in some sense as $k \rightarrow \infty$. Thus, the estimate of $(\alpha, g)$ can be obtained through maximizing

$$
Q_{n}(\alpha, \beta):=\ln \prod_{i=1}^{n} F^{Y_{i}}\left(\alpha^{\top} X_{i}+\beta^{\top} \Phi_{k}\left(Z_{i}\right)\right)\left[1-F\left(\alpha^{\top} X_{i}+\beta^{\top} \Phi_{k}\left(Z_{i}\right)\right)\right]^{1-Y_{i}},
$$

to have $\widehat{\alpha}$ and $\widehat{\beta}$ (hence naturally $\widehat{g}(z)=\widehat{\beta}^{\top} \Phi_{k}(z)$ ). The first order condition gives

$$
\begin{aligned}
& \frac{\partial Q_{n}}{\partial \alpha}=\sum_{i=1}^{n} \frac{\left[Y_{i}-F\left(\alpha^{\top} X_{i}+\beta^{\top} \Phi_{k}\left(Z_{i}\right)\right)\right] F\left(\alpha^{\top} X_{i}+\beta^{\top} \Phi_{k}\left(Z_{i}\right)\right)}{F\left(\alpha^{\top} X_{i}+\beta^{\top} \Phi_{k}\left(Z_{i}\right)\right)\left[1-F\left(\alpha^{\top} X_{i}+\beta^{\top} \Phi_{k}\left(Z_{i}\right)\right)\right]} X_{i}=0, \\
& \frac{\partial Q_{n}}{\partial \beta}=\sum_{i=1}^{n} \frac{\left[Y_{i}-F\left(\alpha^{\top} X_{i}+\beta^{\top} \Phi_{k}\left(Z_{i}\right)\right)\right] F\left(\alpha^{\top} X_{i}+\beta^{\top} \Phi_{k}\left(Z_{i}\right)\right)}{F\left(\alpha^{\top} X_{i}+\beta^{\top} \Phi_{k}\left(Z_{i}\right)\right)\left[1-F\left(\alpha^{\top} X_{i}+\beta^{\top} \Phi_{k}\left(Z_{i}\right)\right)\right]} \Phi_{k}\left(Z_{i}\right)=0,
\end{aligned}
$$

for $i=1, \ldots, n$, which can be viewed as a sample version of moment condition, with $m(\cdot)$ defined properly, $\mathbb{E}\left[m\left(Y_{i}, X_{i}, \Phi_{k}\left(Z_{i}\right), \alpha^{\top} X_{i}, \beta^{\top} \Phi_{k}\left(Z_{i}\right)\right)\right]=0$.

Our strategy for dealing with the specification of model (1.1) is simple. Suppose that $g(\cdot)$ belongs to a suitable Hilbert space. We then expand the function $g(\cdot)$ into an infinite orthogonal series in terms of a basis in the Hilbert space, $\left\{\varphi_{j}(z)\right\}$, say. As a result, $g(z)$ can be approximated by the partial sum $\sum_{j=0}^{k-1} \beta_{j} \varphi_{j}(z)$ in the norm of the space. In this way, the unknown function is completely parameterized, which enables us to estimate the parameter vector $\alpha$ and the function $g(\cdot)$ in model (1.1) simultaneously. This procedure also avoids high level assumptions in our study. By contrast, some high level conditions are engaged in the relevant literature, such as Chen et al. [9] and Han and Phillips [17]. In addition, our approach can be classified as a one-step GMM method, in contrast with the two-step GMM study in the literature that engages an initial estimator. See, for example, Chen and Liao $[8]$.

The rest of the paper is organized as follows. Section 2 gives the estimation procedure; Section 3 provides the asymptotic theory for the estimator proposed in the preceding section; Section 4 studies the over-identification issue, followed by numerical evidence in Section 5; the last section then concludes.

Throughout, $\|\cdot\|$ can be either Euclidean norm for vector or Frobenius norm for matrix, or the norm of functions in function space that would not arise any ambiguity in the context; $\otimes$ denotes Kronecker product for matrices or vectors; := means equal by definition; $I_{r}$ is the identity matrix of dimension $r$. 


\section{Estimation procedure}

The unknown function $g(z)$ can be a vector of functions or a multivariate function. Both of these contexts are useful in practice and they may be dealt with similarly using sieve method. For the sake of easy exposition, however, we suppose in this paper that $g(z)$ is a single multivariate function defined on $\mathbb{Z} \subset \mathbb{R}^{d}$. Let $g(z) \in L^{2}(\mathbb{Z}, \pi(z))=\left\{f(z): \int_{\mathbb{Z}} f^{2}(z) \pi(z) d z<\right.$ $\infty$ \} a Hilbert function space, where $\pi(z)$ is a user-chosen density function on $\mathbb{Z}$. The choice of the density $\pi(z)$ relates to how large the Hilbert space should be, since the thinner the tail of the density is, the larger the space is. For example, $L^{2}\left(\mathbb{R}, 1 /\left(1+z^{2}\right)\right) \subset L^{2}\left(\mathbb{R}, \exp \left(-z^{2}\right)\right)$. An inner product in the Hilbert space is given by $\left\langle f_{1}, f_{2}\right\rangle=\int_{\mathbb{Z}} f_{1}(z) f_{2}(z) \pi(z) d z$, and hence the induced norm $\|f\|=\sqrt{\langle f, f\rangle}$ for any $f_{1}(z), f_{2}(z), f(z) \in L^{2}(\mathbb{Z}, \pi(z))$. Two functions $f_{1}(z), f_{2}(z) \in L^{2}(\mathbb{Z}, \pi(z))$ are called orthogonal if $\left\langle f_{1}, f_{2}\right\rangle=0$, and further are orthonormal if $\left\|f_{1}\right\|=1$ and $\left\|f_{2}\right\|=1$.

The parameter space for model (1.1) is defined as, $\Theta=\left\{(\mathbf{a}, f): \mathbf{a} \in \mathbb{R}^{p}, f \in L^{2}(\mathbb{Z}, \pi(z))\right\}$, which contains the true parameter $(\alpha, g)$ as an interior point.

Assumption 2.1 Suppose that $\left\{\varphi_{j}(\cdot)\right\}$ is a complete orthonormal function sequence in $L^{2}(\mathbb{Z}, \pi(\cdot))$, that is, $\left\langle\varphi_{i}(\cdot), \varphi_{j}(\cdot)\right\rangle=\delta_{i j}$ the Kronecker delta.

Recall that any Hilbert space has a complete orthogonal sequence [see Theorem 5.4.7 in 12, p. 169]. In our setting, although $g(\cdot)$ is multivariate, the orthonormal sequence $\left\{\varphi_{j}(\cdot)\right\}$ can be constructed from the tensor product of univariate orthogonal sequences. Thus, we hereby briefly introduce some existing univariate orthonormal sequences only.

Generally speaking, an orthonormal sequence depends on its support on which it is defined and the density by which the orthogonality is defined. Hermite polynomials form a complete orthogonal sequence on $\mathbb{R}$ with respect to the density $e^{-u^{2}}$; Laguerre polynomials are a complete orthogonal sequence on $[0, \infty)$ with density $e^{-u}$; Legendre polynomials and also orthogonal trigonometric polynomials are complete orthogonal sequence on $[0,1]$ with the uniform density; Chebyshev polynomials are complete orthogonal on $[-1,1]$ with density $1 / \sqrt{1-u^{2}}$. See, e.g. Chapter One of Gautschi [16].

For the function $g(z) \in L^{2}(\mathbb{Z}, \pi(z))$, we may have an infinite orthogonal series expansion:

$$
g(z)=\sum_{j=0}^{\infty} \beta_{j} \varphi_{j}(z), \quad \text { where } \beta_{j}=\left\langle g, \varphi_{j}\right\rangle .
$$

The convergence of (2.1) normally can be understood in the sense of the norm in the space, whereas in the situation where $g(z)$ is smooth, the pointwise sense may hold. For positive integer $k$, define $g_{k}(z)=\sum_{j=0}^{k-1} \beta_{j} \varphi_{j}(z)$ as a truncated series and $\gamma_{k}(z)=\sum_{j=k}^{\infty} \beta_{j} \varphi_{j}(z)$ the residue after truncation. Then, $g_{k}(z) \rightarrow g(z)$ as $k \rightarrow \infty$ in some sense. Note that $g_{k}(z)$ 
is a parameterized version of $g(z)$ in terms of the basis $\left\{\varphi_{j}(z)\right\}$ where only the coefficients remain unknown. This is the main advantage of the sieve method. In addition, the Parseval equality gives $\sum_{j=0}^{\infty} \beta_{j}^{2}=\|g\|^{2}<\infty$, implying the attenuation of the coefficients. For better exposition, denote $\Phi_{k}(z)=\left(\varphi_{0}(z), \ldots, \varphi_{k-1}(z)\right)^{\top}$ and $\beta=\left(\beta_{0}, \ldots, \beta_{k-1}\right)^{\top}$ two $k$-vectors. Thus, $g_{k}(z)=\beta^{\top} \Phi_{k}(z)$.

Our primary goal is to estimate $(\alpha, g(\cdot))$, and the consistency studied below will be defined in terms of a norm given by

$$
\|(\mathbf{a}, f(\cdot))\|=\left(\|\mathbf{a}\|_{E}^{2}+\|f\|_{L^{2}}^{2}\right)^{1 / 2}
$$

where $\|\cdot\|_{E}$ denotes the Euclidean norm on $\mathbb{R}^{p}$ and $\|f\|_{L^{2}}$ signifies the norm on the Hilbert space, of which the subscript may be suppressed whenever there is no ambiguity incurred.

As usual, in order to facilitate an implementation of nonlinear optimization, $\alpha$ should be confined in a compact subset of $\mathbb{R}^{p}$ and the truncated series $g_{k}(z)=\beta^{\top} \Phi_{k}(z)$ of $g$ function should be included in an expanding finite dimensional bounded subsets of $L^{2}(\mathbb{Z}, \pi(z))$. It is noteworthy that in an infinite dimensional space, a bounded subset may not necessarily be a compact set. A detailed discussion on the relationship for the compactness in infinite dimensional space can be found in Chen and Pouzo [10]. Nevertheless, in the case that the function $m$ is linear in the second and the third arguments, such restrictions are not necessary (we shall discuss this in Section 5 using an example).

Assumption 2.2 Suppose that $B_{1 n}$ and $B_{2 n}$ are positive reals diverging with $n$ such that $\alpha$ in model (1.1) is included in $\Theta_{1 n}:=\left\{\mathbf{a} \in \mathbb{R}^{p}:\|\mathbf{a}\| \leq B_{1 n}\right\}$ and for sufficient large $n, g_{k}(z)$ is included in $\Theta_{2 n}:=\left\{\mathbf{b}^{\top} \Phi_{k}(z):\|\mathbf{b}\| \leq B_{2 n}\right\}$.

Here, unlike in a general single-index model, we do not require $\|\alpha\|=1$ for identification. This is because the function $m(\cdot)$ is known and hence we are able to identify any scaling for $\alpha$. It is also a convention on computation that the true parameter is assumed to be contained within a bounded set [see 22, p. 1569], whereas the difference is that in this paper we allow the bounds of $\alpha$ to diverge with the sample size since the dimensionality of $\alpha$ grows to infinity.

Meanwhile, since $\left\|g_{k}(z)\right\|=\|\beta\| \leq\|g\|$ it is clear that there exists an integer $n_{0}$ such that $g_{k}(z) \in \Theta_{2 n}$ for all $n \geq n_{0}$. Similar to the orthogonal expansion in (2.1), for any $f(z) \in L^{2}(\mathbb{Z}, \pi(z)), f(z)$ can be approximated by $\sum_{j=0}^{k-1} b_{j} \varphi_{j}(z)=\mathbf{b}^{\top} \Phi_{k}(z)$ arbitrarily in the sense of norm, where $b_{j}$ and $\mathbf{b}$ are defined similarly to $\beta_{j}$ and $\beta$, respectively. This means that $\Theta_{2 n}$ is approximating the function space with the increase of the sample size. Thus, the parametric space can be approximated by $\Theta_{n}=\Theta_{1 n} \otimes \Theta_{2 n}$ as $n \rightarrow \infty$. In the literature, $\Theta_{2 n}$ is the so-called linear sieve space. More importantly, $\Theta_{n}$ is bounded and compact. The above setting is similar to but broader than that in Newey and Powell [22]. 
We rewrite the moment condition (1.1) as

$$
\mathbb{E}\left[m\left(V_{i}, \alpha^{\top} X_{i}, \beta^{\top} \Phi_{k}\left(Z_{i}\right)+\gamma_{k}\left(Z_{i}\right)\right)\right]=0
$$

where $\gamma_{k}(\cdot)$ is negligible for large $k$. We estimate $\alpha$ and $\beta$ by

$$
\begin{gathered}
(\widehat{\alpha}, \widehat{\beta})=\underset{\mathbf{a} \in \mathbb{R}^{p}, \mathbf{b} \in \mathbb{R}^{k}}{\operatorname{argmin}}\left\|M_{n}(\mathbf{a}, \mathbf{b})\right\|^{2}, \quad \text { subject to }\|\mathbf{a}\| \leq B_{1 n} \text { and }\|\mathbf{b}\| \leq B_{2 n}, \\
\quad \text { where } M_{n}(\mathbf{a}, \mathbf{b})=\frac{1}{\sqrt{q}} \frac{1}{n} \sum_{i=1}^{n} m\left(V_{i}, \mathbf{a}^{\top} X_{i}, \mathbf{b}^{\top} \Phi_{k}\left(Z_{i}\right)\right) .
\end{gathered}
$$

Here, the involvement of $q$ in $M_{n}(\mathbf{a}, \mathbf{b})$ takes into account the divergence of the dimension of the $m$ function in order to avoid that $\left\|M_{n}(\mathbf{a}, \mathbf{b})\right\|$ could be large even if each element is small when we had not put $q$ into $M_{n}(\mathbf{a}, \mathbf{b})$. However, this issue does not matter when the vector-valued $m$ function has a fixed dimension. In addition, from the proof of the theorems below, the term $\sqrt{q}$ in $M_{n}(\mathbf{a}, \mathbf{b})$ can be replaced by some appropriately chosen function of $q$, which normalizes the divergence of the norm of $M_{n}(\mathbf{a}, \mathbf{b})$. For the sake of simplicity, we take $\sqrt{q}$ in this paper. Then, naturally define

$$
\widehat{g}(z)=\widehat{\beta}^{\top} \Phi_{k}(z)
$$

for any $z \in \mathbb{Z}$ as an estimator of $g(z)$. In the next section we establish consistency of this estimator in the sense that $\|(\widehat{\theta}-\theta, \widehat{g}(z)-g(z))\| \rightarrow_{P} 0$ as $n \rightarrow \infty$ where the norm is defined in $(2.2)$.

\section{Asymptotic theory}

\subsection{Consistency}

Before starting our asymptotic theory, we need to state some necessary assumptions.

Assumption 3.1 Suppose that: (a) for each $n,\left\{V_{i}, X_{i}^{\top}, Z_{i}^{\top}\right\}_{1}^{n}$ is an independent and identically distributed sequence; (b) for the density $f_{Z}(z)$ of $Z_{1}, f_{Z}(z) \leq C \pi(z)$ on the support $\mathbb{Z}$ of $Z_{1}$ for some constant $C$; (c) the function $m(\cdot, \cdot, \cdot)$ is continuous in the second and third arguments; (d) $q(n)-p(n) \geq k$.

The i.i.d. property in Assumption 3.1.a simplifies the presentation and some of the calculations, although it is possible to relax it a dependent data setting. About Assumption 3.1.b, the relation between the densities of the variable $Z_{1}$ and the function space is widely used in the literature. See, e.g. Condition A.2 and Proposition 2.1 of Belloni et al. [2, p. 347]. For Assumption 3.1.c, the continuity of the $m$ function in the arguments where the 
parameters are involved is weak. In Assumption 3.1.d we allow for possible overidentification of the parameter vector in the moment conditions, and we shall discuss this issue further in the next section.

Assumption 3.2 Suppose that there is a unique function $g(\cdot) \in L^{2}(\mathbb{Z}, \pi(z))$ and for each $n$ there is a unique vector $\alpha \in \mathbb{R}^{p}$ such that model (1.1) is satisfied. In other words, for any $\delta>0$, there is an $\epsilon>0$ such that

$$
\inf _{\substack{(\mathbf{a}, f) \in \Theta \\\|(\mathbf{a}-\alpha, f-g)\| \geq \delta}} q^{-1}\left\|\mathbb{E} m\left(V_{i}, \mathbf{a}^{\top} X_{i}, f\left(Z_{i}\right)\right)\right\|^{2}>\epsilon .
$$

It is quite standard in the literature to assume such a uniqueness condition. Here again, the squared norm is scaled down by its dimension due to the same reason as in the formulation of $M_{n}$ in the last section, for which we do not mention repeatedly in what follows whenever the norm is scaled.

Assumption 3.3 Suppose that for each $n$, there is a measurable positive function $A(V, X, Z)$ such that

$$
q^{-1 / 2}\left\|m\left(V, \mathbf{a}_{1}^{\top} X, f_{1}(Z)\right)-m\left(V, \mathbf{a}_{2}^{\top} X, f_{2}(Z)\right)\right\| \leq A(V, X, Z)\left[\left\|\mathbf{a}_{1}-\mathbf{a}_{2}\right\|+\left|f_{1}(Z)-f_{2}(Z)\right|\right]
$$

for any $\left(a_{1}, f_{1}\right),\left(a_{2}, f_{2}\right) \in \Theta$, where $(V, X, Z)$ is any realization of $\left(V_{i}, X_{i}, Z_{i}\right)$ and the function A satisfies that $\max _{i \geq 1} \mathbb{E}\left[A^{2}\left(V_{i}, X_{i}, Z_{i}\right)\right]<\infty$ uniformly in $n$.

This assumption is a kind of Lipschitz condition. The positive function $A(V, X, Z)$ may be viewed as the upper bound of the norm of the partial derivatives of $q^{-1 / 2} m\left(V, \mathbf{a}^{\top} X, w\right)$ with respect to vector a and scalar $w$, respectively, and thus the condition is fulfilled if the second moment of $A(V, X, Z)$ is bounded. The assumption guarantees the approximation $m\left(V_{i}, \alpha^{\top} X_{i}, \beta^{\top} \Phi_{k}\left(Z_{i}\right)\right)$ to $m\left(V_{i}, \alpha^{\top} X_{i}, g\left(Z_{i}\right)\right)$, because

$$
\begin{aligned}
& \left\|m\left(V_{i}, \alpha^{\top} X_{i}, \beta^{\top} \Phi_{k}\left(Z_{i}\right)\right)-m\left(V_{i}, \alpha^{\top} X_{i}, g\left(Z_{i}\right)\right)\right\| \\
\leq & A\left(V_{i}, X_{i}, Z_{i}\right)\left\|g\left(Z_{i}\right)-\beta^{\top} \Phi_{k}\left(Z_{i}\right)\right\|=O_{P}(1)\left\|\gamma_{k}(z)\right\|=o_{P}(1)
\end{aligned}
$$

by virtue of Assumption 3.1(b). Also, it ensures that $\left\|\mathbb{E} m\left(V_{i}, \alpha^{\top} X_{i}, \beta^{\top} \Phi_{k}\left(Z_{i}\right)\right)\right\|=o(1)$ since $\mathbb{E} m\left(V_{i}, \alpha^{\top} X_{i}, g\left(Z_{i}\right)\right)=0$. More importantly,

$$
\begin{aligned}
& q^{-1} \mathbb{E}\left\|m\left(V_{i}, \mathbf{a}^{\top} X_{i}, f\left(Z_{i}\right)\right)\right\|^{2} \\
\leq & 2 q^{-1} \mathbb{E}\left\|m\left(V_{i}, 0,0\right)\right\|+2 \mathbb{E}\left[A\left(V_{i}, X_{i}, Z_{i}\right)^{2}\right]\left[\|\mathbf{a}\|^{2}+\mathbb{E} f\left(Z_{i}\right)^{2}\right]=O\left(B_{1 n}^{2}+B_{2 n}^{2}\right)
\end{aligned}
$$

uniformly on $(\mathbf{a}, f) \in \Theta_{n}$.

Theorem 3.1 (Consistency). In addition to Assumptions 2.1-2.2 and 3.1-3.3, suppose that $B_{1 n}^{2}+B_{2 n}^{2}=o(n)$. Then, we have $\|(\widehat{\alpha}-\alpha, \widehat{g}(z)-g(z))\| \rightarrow_{P} 0$ as $n \rightarrow \infty$.

The proof is given in Appendix B. 


\subsection{Limit distributions of the estimator}

As the dimension of $\alpha$ diverges, we may not be able to establish a limit distribution for $\widehat{\alpha}-\alpha$. Instead, we shall aim at some finite dimensional transformations of $\alpha$ and functionals of $g(z)$, by involving the plug-in estimators for these functionals.

Let $\mathscr{L}$ be a linear transformation from $\mathbb{R}^{p} \mapsto \mathbb{R}^{r}$ with $r \geq 1$ fixed, and $\mathscr{F}=\left(\mathscr{F}_{1}, \ldots, \mathscr{F}_{s}\right)^{\top}$ with fixed $s$ be a vector of functionals on $L^{2}(\mathbb{Z}, \pi(z))$. Normally, the transformation $\mathscr{L}$ can be understood as an $r \times p$ matrix with rank $r$, while in the literature one usually takes $r=1$. See, e.g. Theorem 4.2 in Belloni et al. [2, p. 352] and several results such as Theorems 2 and 6 in Chang et al. [6]. Moreover, the elements of $\mathscr{F}$ can be, as described in Newey $[21, \mathrm{p} .151]$, the integral of $\ln [g(z)]$ on some interval which stands for consumer's surplus in microeconomics, for example. Other examples include partial derivative function, average partial derivative function and conditional partial derivative.

Thus, we shall consider the limit distributions of $\mathscr{L}(\widehat{\alpha})-\mathscr{L}(\alpha)$ and $\mathscr{F}(\widehat{g})-\mathscr{F}(g)$.

Assumption 3.4 Suppose that the $m$ function is differentiable with respect to its second and third arguments up to the third order. Let the $g$ function be smooth such that Assumption A.2 in Appendix A is satisfied.

The differentiability of the $m$ function up to the third order makes the derivation of the asymptotic distribution below much simpler than in some papers in the literature since it enables us to expand the score function where the terms with higher order than the Hessian matrix can be ignored. Certainly, this condition can be relaxed to have the derivatives of up to the second order but for simplicity we retain it. It is well known that certain smoothness order of the $g$ function is required to get rid of the truncation residues. Such a requirement is implicitly spelt out by Assumption A.2.

To investigate the asymptotics, denote the Score and Hessian functions

$$
S_{n}(\mathbf{a}, \mathbf{b}):=\left(\begin{array}{c}
\frac{\partial}{\partial \mathbf{a}} \\
\frac{\partial}{\partial \mathbf{b}}
\end{array}\right)\left\|M_{n}(\mathbf{a}, \mathbf{b})\right\|^{2}, \quad H_{n}(\mathbf{a}, \mathbf{b}):=\left(\begin{array}{cc}
\frac{\partial^{2}}{\partial \mathbf{a} \partial \mathbf{a}^{\top}} & \frac{\partial^{2}}{\partial \mathbf{a} \partial \mathbf{b}^{\top}} \\
\frac{\partial^{2}}{\partial \mathbf{b} \partial \mathbf{a}^{\top}} & \frac{\partial^{2}}{\partial \mathbf{b} \partial \mathbf{b}^{\top}}
\end{array}\right)\left\|M_{n}(\mathbf{a}, \mathbf{b})\right\|^{2} .
$$

Under certain conditions, the asymptotic behavior of $H_{n}(\alpha, \beta)$ and $S_{n}(\alpha, \beta)$ is given by Lemmas A.2 and A.3 in Appendix A.

Recall the Fréchet derivative operator for an operator from one Banach space to another. Note that it is a bounded linear operator. In the current case, the Fréchet derivative of $\mathscr{F}$ at $g(\cdot)$ is an $s$-vector of functionals, denoted by $\mathscr{F}^{\prime}(g)$, such that

$$
\mathscr{F}(\widehat{g})-\mathscr{F}(g)=\mathscr{F}^{\prime}(g)(\widehat{g}-g)+\lambda(g, \widehat{g}-g),
$$

where $\lambda(g, \widehat{g}-g)=o(\|\widehat{g}-g\|)$. 
Theorem 3.2 (Normality). Let Assumptions 2.1-2.2, 3.1-3.4 and A.1-A.3 (given in Appendix A) hold. Then

$$
\sqrt{n} \Sigma_{n}^{-1}\left(\begin{array}{c}
\mathscr{L}(\widehat{\alpha})-\mathscr{L}(\alpha) \\
\mathscr{F}(\widehat{g})-\mathscr{F}(g)
\end{array}\right) \stackrel{d}{\rightarrow} N\left(0, I_{r+s}\right)
$$

as $n \rightarrow \infty$ provided that $\sqrt{n} \Sigma_{n}^{-1}\left(\mathbf{0}_{r}^{\top}, \mathscr{F}^{\prime}(g) \gamma_{k}(z)^{\top}\right)^{\top}=o(1)$, where $\Sigma_{n}$ is given by the square root of

$$
\begin{aligned}
& \Sigma_{n}^{2}:=\Gamma_{n}\left[\Psi_{n} \Psi_{n}^{\top}\right]^{-1} \Psi_{n} \Xi_{n} \Psi_{n}^{\top}\left[\Psi_{n} \Psi_{n}^{\top}\right]^{-1} \Gamma_{n}^{\top}, \quad \text { in which } \\
& \Gamma_{n}:=\left(\begin{array}{cc}
\mathscr{L} & 0 \\
0 & \mathscr{F}^{\prime}(g) \Phi_{k}(\cdot)^{\top}
\end{array}\right)_{(r+s) \times(p+k)}, \\
& \Xi_{n}:=\mathbb{E}\left[m\left(V_{1}, \alpha^{\top} X_{1}, g\left(Z_{1}\right)\right) m\left(V_{1}, \alpha^{\top} X_{1}, g\left(Z_{1}\right)\right)^{\top}\right]_{q \times q}, \\
& \Psi_{n}:=\mathbb{E}\left(\begin{array}{c}
\frac{\partial}{\partial u} m\left(V_{1}, \alpha^{\top} X_{1}, g\left(Z_{1}\right)\right)^{\top} \otimes X_{1} \\
\frac{\partial}{\partial w} m\left(V_{1}, \alpha^{\top} X_{1}, g\left(Z_{1}\right)\right)^{\top} \otimes \Phi_{k}\left(Z_{1}\right)
\end{array}\right)_{(p+k) \times q},
\end{aligned}
$$

in which $u$ and $w$ stand for the second and the third arguments of the vector function $m(v, u, w)$, respectively.

The proof is given in Appendix B. Apart from the diverging dimensions of $\Psi_{n}$ and $\Xi_{n}$ and the use of the transformation $\mathscr{L}$ and the functional $\mathscr{F}$, the form of the covariance matrices $\Sigma_{n}^{2}$ is exactly the same as in the literature such as Hansen [18], Pakes and Pollard [24] and Chen et al. [9].

The requirement of $\sqrt{n} \Sigma_{n}^{-1}\left(\mathbf{0}_{r}^{\top}, \mathscr{F}^{\prime}(g) \gamma_{k}(z)^{\top}\right)^{\top}=o(1)$ is an undersmoothing condition, playing a similar role to its counterpart in the literature, see, for example, the condition $\sqrt{n} V_{K}^{-1} K^{-p / d}=o(1)$ in Corollary 3.1 of Chen and Christensen [7, p. 454] and Comment 4.3 of Belloni et al. [2]. If $r=1$, the transformation $\mathscr{L}$ will transform the vector $\alpha$ into a scalar, $\mathscr{L}(\alpha)=a_{0}^{\top} \alpha$, for some $a_{0} \in \mathbb{R}^{p}$ and $a_{0} \neq 0$. This is the case commonly encountered in the literature. See, e.g. Chang et al. [6] and Belloni et al. [2].

It is clear that the convergence order of $\mathscr{L}(\widehat{\alpha}-\alpha)$ is $n^{-1 / 2}$, while that of $\mathscr{F}(\widehat{g})-\mathscr{F}(g)$ is proportional to $\left(\mathscr{F}^{\prime}(g) \Phi_{k}(z)^{\top} \mathscr{F}^{\prime \top} \Phi_{k}(z)\right)^{1 / 2} n^{-1 / 2}$, which is similar to the result in Theorem 2 of Newey [21]. Here, the matrix in the front of $n^{-1 / 2}$ is of dimension $s \times s$ associated with the derivative of functional $\mathscr{F}$. To understand how it affects the rate, consider a special case that $s=1$ and $\mathscr{F}(g)=g(z)$ for some particular $z$, implying $\mathscr{F}(\widehat{g})-\mathscr{F}(g)=\widehat{g}(z)-g(z)$ and $\mathscr{F}^{\prime}(g) \equiv 1$. Then, the matrix is a scalar and the rate becomes $\left\|\Phi_{k}(z)\right\| n^{-1 / 2}$, which coincides with the rates of convergence in the literature, see, for example, Dong and Linton [11].

The limiting normal distribution involves unknown parameters in the matrix $\Sigma_{n}$. In practice one would need a consistent estimator for this matrix. It is easily seen that the 
consistent estimator, $\widehat{\Sigma}_{n}$, of $\Sigma_{n}$ can be obtained if we replace $\alpha$ and $g(\cdot)$ in $\Sigma_{n}$ by $\widehat{\alpha}$ and $\widehat{g}(\cdot)$, as well as the expectations in $\Xi_{n}$ and $\Psi_{n}$ by their sample versions. More precisely, let

$$
\widehat{\Sigma}_{n}^{2}=\widehat{\Gamma}_{n}\left[\widehat{\Psi}_{n} \widehat{\Psi}_{n}^{\top}\right]^{-1} \widehat{\Psi}_{n} \widehat{\Xi}_{n} \widehat{\Psi}_{n}^{\top}\left[\widehat{\Psi}_{n} \widehat{\Psi}_{n}^{\top}\right]^{-1} \widehat{\Gamma}_{n}^{\top},
$$

where $\widehat{\Gamma}_{n}$ is $\Gamma_{n}$ with replacement of $\mathscr{F}^{\prime}(g)$ by $\mathscr{F}^{\prime}(\widehat{g})$ and

$$
\begin{aligned}
& \widehat{\Xi}_{n}:=\frac{1}{n} \sum_{i=1}^{n}\left[m\left(V_{i}, \widehat{\alpha}^{\top} X_{i}, \widehat{g}\left(Z_{i}\right)\right) m\left(V_{i}, \widehat{\alpha}^{\top} X_{i}, \widehat{g}\left(Z_{i}\right)\right)^{\top}\right] \\
& \widehat{\Psi}_{n}:=\frac{1}{n} \sum_{i=1}^{n}\left(\begin{array}{c}
\frac{\partial}{\partial u} m\left(V_{i}, \widehat{\alpha}^{\top} X_{i}, \widehat{g}\left(Z_{i}\right)\right)^{\top} \otimes X_{i} \\
\frac{\partial}{\partial w} m\left(V_{i}, \widehat{\alpha}^{\top} X_{i}, \widehat{g}\left(Z_{i}\right)\right)^{\top} \otimes \Phi_{k}\left(Z_{i}\right)
\end{array}\right) .
\end{aligned}
$$

It is readily seen that $\widehat{\Sigma}_{n}-\Sigma_{n} \rightarrow_{P} 0$ as $n \rightarrow \infty$.

If a weight matrix is used in the minimization, the efficiency of the limit theorem may be improved. Let $W_{n}=W_{n}(\alpha, \beta)$ be a $q \times q$ positive definite matrix depending on the parameter and the data used in $M_{n}$. Then, $\left\|M_{n}(\mathbf{a}, \mathbf{b})\right\|^{2}$, which measures the metric of $M_{n}(\mathbf{a}, \mathbf{b})$ from zero, can be substituted by $M_{n}(\mathbf{a}, \mathbf{b})^{\top} W_{n}(\mathbf{a}, \mathbf{b}) M_{n}(\mathbf{a}, \mathbf{b})$ in the minimization of (2.4), which is also a measure of the metric for the vector $M_{n}(\mathbf{a}, \mathbf{b})$ from zero but in terms of the weight matrix $W_{n}$. Meanwhile, $\left\|M_{n}(\mathbf{a}, \mathbf{b})\right\|^{2}$ can be viewed as a special case that $W_{n}$ is the identity matrix. Definitely, $W_{n}$ can not be close to singular to eschew the possibility that $M_{n}(\mathbf{a}, \mathbf{b})^{\top} W_{n}(\mathbf{a}, \mathbf{b}) M_{n}(\mathbf{a}, \mathbf{b})$ may be close to zero when $(\mathbf{a}, \mathbf{b})$ is far from $(\alpha, \beta)$.

Proposition 3.1. Suppose that the eigenvalues of $W_{n}$ are bounded away from zero and above from infinity uniformly in $n$, and that $\sup _{\|(\mathbf{a}-\alpha, \mathbf{b}-\beta)\|<\delta_{n}}\left\|W_{n}(\mathbf{a}, \mathbf{b})-W_{n}\right\|=o_{P}(1)$ with $\delta_{n}=$ $o(1)$ when $n$ large. Let $(\widetilde{\alpha}, \widetilde{\beta})$ be the minimizer of $M_{n}(\mathbf{a}, \mathbf{b})^{\top} W_{n}(\mathbf{a}, \mathbf{b}) M_{n}(\mathbf{a}, \mathbf{b})$ and define $\widetilde{g}(z)=\Phi_{k}(z)^{\top} \widetilde{\beta}$.

Then, (1) Under the same conditions in Theorem 3.1, the consistency of the weighted estimator holds; (2) Under the same conditions the normality for the weighted estimator in Theorem 3.2 holds with $\Sigma_{n}^{2}$ replaced by

$$
\Gamma_{n}\left[\Psi_{n} W_{n} \Psi_{n}^{\top}\right]^{-1} \Psi_{n} W_{n} \Xi_{n} W_{n} \Psi_{n}^{\top}\left[\Psi_{n} W_{n} \Psi_{n}^{\top}\right]^{-1} \Gamma_{n}^{\top}
$$

(3) If $W_{n}=\Xi_{n}^{-1}$, the optimal covariance matrices is obtained, $\Gamma_{n}\left[\Psi_{n} \Xi_{n}^{-1} \Psi_{n}^{\top}\right]^{-1} \Gamma_{n}^{\top}$.

The proof is given in Appendix B. Here, the optimal covariance is in the sense that

$$
\Gamma_{n}\left[\Psi_{n} W \Psi_{n}^{\top}\right]^{-1} \Psi_{n} W \Xi_{n} W \Psi_{n}^{\top}\left[\Psi_{n} W \Psi_{n}^{\top}\right]^{-1} \Gamma_{n}^{\top} \geq \Gamma_{n}\left[\Psi_{n} \Xi_{n}^{-1} \Psi_{n}^{\top}\right]^{-1} \Gamma_{n}^{\top}
$$

for all $W$ satisfying the conditions in the proposition. In practice, both $\Xi_{n}$ and $\Psi_{n}$ can be replaced by their sample versions of (3.1) and (3.2), so that the optimal covariance matrices 
are easily estimable. Nonetheless, in order to obtain an optimal estimator one will need to implement a two-step estimation method, as has normally been done in the literature, that is, at the first step minimizing $\left\|M_{n}(\mathbf{a}, \mathbf{b})\right\|^{2}$ to have $\widehat{\alpha}$ and $\widehat{g}(\cdot)$ that are used to construct $\widehat{W}_{n}$; then at the second step one may minimize $M_{n}(\mathbf{a}, \mathbf{b})^{\top} \widehat{W}_{n} M_{n}(\mathbf{a}, \mathbf{b})$ to have a pair of optimal estimators, $(\widetilde{\alpha}, \widetilde{g}(\cdot))$.

In addition to their earlier work by Cattaneo et al. [4] on a partially linear model, Cattaneo et al. [5] recently develop heteroskedasticity robust inference methods for the finite dimensional parameters of a linear model in the presence of a large number of linearly estimated nuisance parameters in the case where essentially $p$ is fixed but $K(n) \propto n$. In this case, the function $g(\cdot)$ is not consistently estimated. We interpret the differencing approach proposed by Yatchew [29] and Yatchew [30] for the partially linear model as being similar to this, except that Cattaneo et al. [5] allow for heteroskedasticity and for a more complex type of nuisance component. In our methodology we pay equal attention to the function $g$, which itself can be of interest, see for example, Engle et al. [13]; Robinson [28]; Gao and Liang [14]; Gao and Shi [15] and Härdle et al. [19]. Our methodology is also robust to conditional heteroskedasticity.

\section{Statistical inference}

\subsection{Test of over-identification}

Hansen [18] proposes the J-test for over-identification in the situation where both $p$ and $q$ are fixed but $q>p$. This J-test has an asymptotic $\chi_{q-p}^{2}$ distribution. In the case where an unknown infinite dimensional parameter is involved but both $p$ and $q$ are still fixed with $q>p$, Chen and Liao [8] establish a statistic for over-identification testing that has an $F$ distribution in large samples. As far as we are aware, the test statistic proposed below seems a new one in the literature.

Because we also face an over-identification situation where $q(n)-p(n) \rightarrow \infty$, it is crucial to test whether the moment restrictions are valid by investigating the following hypotheses:

$$
H_{0}: \quad \mathbb{E}\left[m\left(V_{i}, \alpha^{\top} X_{i}, g\left(Z_{i}\right)\right)\right]=0 \text { for some }(\alpha, g) \in \Theta
$$

versus

$$
H_{1}: \quad \mathbb{E}\left[m\left(V_{i}, \mathbf{a}^{\top} X_{i}, h\left(Z_{i}\right)\right)\right] \neq 0 \text { for any }(\mathbf{a}, h) \in \Theta,
$$

where $\Theta$ is defined in Section 2 . 
Define, for $\mathbf{a} \in \mathbb{R}^{p}, \mathbf{b} \in \mathbb{R}^{k}$ and any $\kappa \in \mathbb{R}^{q}$ such that $\|\kappa\|=1$,

$$
L_{n}(\mathbf{a}, \mathbf{b} ; \kappa)=\frac{1}{D_{n}(\mathbf{a}, \mathbf{b} ; \kappa)} \sum_{i=1}^{n} \kappa^{\top} m\left(V_{i}, \mathbf{a}^{\top} X_{i}, \mathbf{b}^{\top} \Phi_{k}\left(Z_{i}\right)\right),
$$

where $D_{n}(\mathbf{a}, \mathbf{b} ; \kappa)=\left(\sum_{i=1}^{n}\left[\kappa^{\top} m\left(V_{i}, \mathbf{a}^{\top} X_{i}, \mathbf{b}^{\top} \Phi_{k}\left(Z_{i}\right)\right)\right]^{2}\right)^{1 / 2}$.

Under the null, by the procedure in Section 2 and Assumptions in Theorem 3.1, we have the consistent estimator $(\widehat{\alpha}, \widehat{g})$. The statistic $L_{n}(\widehat{\alpha}, \widehat{\beta} ; \kappa)$ can be used to detect $H_{0}$ against $H_{1}$, as shown in Theorems 4.1 and 4.2 below. It is noteworthy that this test, as clearly indicated from the proof, is also workable for the conventional moment restriction models with fixed $p$ and $q$. Before showing the asymptotic distribution under the null and the consistency under the alternative for the test statistic, we introduce some necessary assumptions.

Assumption 4.1 Let $\bar{m}_{n}^{*}(\widehat{\alpha}, \widehat{g} ; \kappa)=o_{P}(1)$ when $n \rightarrow \infty$, where we denote $\bar{m}_{n}^{*}(\mathbf{a}, f ; \kappa)=$ $n^{-1 / 2} \sum_{i=1}^{n} E\left[\kappa^{\top} m\left(V_{i}, \mathbf{a}^{\top} X_{i}, f\left(Z_{i}\right)\right)\right]$ for $(\mathbf{a}, f) \in \Theta$ and $\kappa$ such that $\|\kappa\|=1$.

Assumption 4.2 Suppose that (i) $q^{2} p=o(n)$ and $q^{2} k=o(n)$; and (ii) $\sup _{z} \gamma_{k}^{2}(z)=$ $o\left(q^{-1}\right)$ as, along with $n \rightarrow \infty, k, p, q \rightarrow \infty$.

These are technical requirements. Noting $E\left[m\left(V_{i}, \alpha^{\top} X_{i}, g\left(Z_{i}\right)\right)\right]=0$, Assumption 4.1 requires that $E\left[m\left(V_{i}, \mathbf{a}^{\top} X_{i}, f\left(Z_{i}\right)\right)\right]$ drops to zero very quickly when $(\mathbf{a}, f)$ approaches $(\alpha, g)$. This in spirit is the same as Assumption 3.2 but here it is a sample version and the decay of the expectation needs a certain rate. Similar assumption is also imposed by equation (4.9) of Andrews [1, p. 58] and equation (5.2) of Belloni et al. [3, p. 774]. Assumption 4.2 (i) stipulates the relationships for $p, q, k$ with $n$ when they are diverging, while Assumption 4.2(ii) imposes a decay rate for the residue $\gamma_{k}^{2}(z)$ uniformly for all $z$ not slower than $o\left(q^{-1}\right)$. This particularly is satisfied for the case where $z$ is located in some compact set in many situations given that the $g$ function is sufficiently smooth.

Theorem 4.1. Suppose that there is no zero function in the vector $m$ of functions. Let Assumptions 4.1-4.2 hold, under $H_{0}$ and the conditions in Theorems 3.1 and 3.2 remain true. For any $\kappa \in \mathbb{R}^{q}$ such that $\|\kappa\|=1$,

$$
L_{n}(\widehat{\alpha}, \widehat{\beta} ; \kappa) \rightarrow_{D} N(0,1)
$$

as $n \rightarrow \infty$, where $(\widehat{\alpha}, \widehat{\beta})$ is the estimator given by $(2.4)$.

Notice that if there is a zero function in $m, \kappa^{\top} m$ can be a zero function for some particular $\kappa$. Thus, the requirement on the nonzero function is trivial. The theorem shows the normality of the proposed statistic that enables us to make statistical inference. 
Theorem 4.2. Suppose that the eigenvalues of $E\left[m\left(V_{1}, \mathbf{a}^{\top} X_{1}, h\left(Z_{1}\right)\right) m\left(V_{1}, \mathbf{a}^{\top} X_{1}, h\left(Z_{1}\right)\right)^{\top}\right]$ are uniformly bounded away from zero and infinity in $n$ and $(\mathbf{a}, h) \in \Theta$. Under $H_{1}$, suppose further that there exists a positive sequence $\delta_{n}$ such that $\inf _{(\mathbf{a}, h) \in \Theta}\left\|E\left[m\left(V_{i}, \mathbf{a}^{\top} X_{i}, h\left(Z_{i}\right)\right)\right]\right\| \geq$ $\delta_{n}$ and $\liminf _{n \rightarrow \infty} \sqrt{n} \delta_{n}=\infty$. Then, for any vector $\mathbf{a}$ and $\mathbf{b}$, there exists some $\kappa^{*} \in \mathbb{R}^{q}$ such that $\left\|\kappa^{*}\right\|=1$ and $L_{n}\left(\mathbf{a}, \mathbf{b} ; \kappa^{*}\right) \rightarrow_{P} \infty$, as $n \rightarrow \infty$.

The condition on the eigenvalues is broadly adopted in the literature, see, e.g. Chang et al. [6] and Belloni et al. [2]. In the special case where $\delta_{n}=\delta$, the condition that $\liminf _{n \rightarrow \infty} \sqrt{n} \delta_{n}=\infty$ is satisfied automatically, and this is the most commonly used assumption in the literature, see, equation (24) of Chang et al. [6, p. 290]. However, we allow for $\delta_{n} \rightarrow 0$ with a rate slower than $n^{-1 / 2}$. This means that the strongest signal $\left(\delta_{n}=\delta\right)$ can be weakened $\left(\delta_{n} \rightarrow 0\right)$ when our test statistic is used.

\subsection{Wald test}

The normality in Theorem 3.2 may be used for Wald test to detect, for example, $\mathcal{H}_{10}$ : $\mathscr{L} \alpha=R$ against $\mathcal{H}_{11}: \mathscr{L} \alpha \neq R$ for some transformation $\mathscr{L}$ of $r \times p$ matrix and $r$-vector $R$; $\mathcal{H}_{20}: \mathscr{F}(g)=S$ against $\mathcal{H}_{21}: \mathscr{F}(g) \neq S$ for some $s$-vector functional $\mathscr{F}$ and $s$-vector $S$.

Let $\widehat{\Sigma}_{1 n}^{2}=\left(\begin{array}{ll}I_{r} & \mathbf{0}_{r \times s}\end{array}\right) \widehat{\Sigma}_{n}^{2}\left(\begin{array}{ll}I_{r} & \mathbf{0}_{r \times s}\end{array}\right)^{\top}$ and $\widehat{\Sigma}_{2 n}^{2}=\left(\begin{array}{ll}\mathbf{0}_{s \times r} & I_{s}\end{array}\right) \widehat{\Sigma}_{n}^{2}\left(\begin{array}{ll}\mathbf{0}_{s \times r} & I_{s}\end{array}\right)^{\top}$. Then, Theorem 3.2 implies that, under $\mathcal{H}_{10}$ and $\mathcal{H}_{20}$, respectively, we have

$$
n(\mathscr{L} \widehat{\alpha}-R)^{\top} \widehat{\Sigma}_{1 n}^{-1}(\mathscr{L} \widehat{\alpha}-R) \stackrel{d}{\rightarrow} \chi^{2}(r), \text { and } n(\mathscr{F}(\widehat{g})-S)^{\top} \widehat{\Sigma}_{2 n}^{-1}(\mathscr{F}(\widehat{g})-S) \stackrel{d}{\rightarrow} \chi^{2}(s),
$$

respectively, when $n \rightarrow \infty$.

\section{$5 \quad$ Simulation experiments}

In this section, we are about to investigate the performance of the proposed estimators in finite sample situation.

Example 5.1. This experiment uses the model in Example 1.1 of Section 1. Let vector $X_{i}=\left(X_{1 i}, X_{2 i}^{\top}\right)^{\top}$ where $X_{1 i}$ assumes 1 and -1 with probability $1 / 2$, respectively, $X_{2 i} \sim$ $N\left(0, \Sigma_{p-1}\right)$, where $\Sigma_{p-1}=\left(\sigma_{i, j}\right)_{(p-1) \times(p-1)}$ with $\sigma_{i, i}=1, \sigma_{i, j}=0.3$ for $|i-j|=1$ and $\sigma_{i, j}=0$ for $|i-j|>1$. Here, the first component of $X_{i}$ is a discrete variable with which we intend to show that our theoretical results do not confine in continuous variables. Let $Z_{i}$ be uniformly distributed on $(0,1)$.

Suppose that $\mathbb{E}\left[Y_{i}-\alpha^{\top} X_{i}-g\left(Z_{i}\right) \mid W_{i}\right]=0$ with $W_{i}=Z_{i}$, and $g(\cdot) \in L^{2}[0,1]=\{u(r)$ : $\left.\int_{0}^{1} u^{2}(r) d r<\infty\right\}$. Let $\varphi_{0}(r) \equiv 1$, and for $j \geq 1, \varphi_{j}(r)=\sqrt{2} \cos (\pi j r)$. Then, $\left\{\varphi_{j}(r)\right\}$ is an orthonormal basis in the Hilbert space $L^{2}[0,1]$. In the experiment, put $\alpha=(0.4,0.1,0, \cdots, 0)^{\top} \in$ $\mathbb{R}^{p}$ and $g(z)=z^{2}+\sin (z)$. 
Denote $m\left(V_{i}, \alpha^{\top} X_{i}, g\left(Z_{i}\right)\right)=\left(Y_{i}-\alpha^{\top} X_{i}-g\left(Z_{i}\right)\right) \Phi_{q}\left(Z_{i}\right)$ where $V_{i}=\left(Y_{i}, W_{i}\right), W_{i}=Z_{i}$ and $\Phi_{q}(\cdot)=\left(\varphi_{0}(\cdot), \cdots, \varphi_{q-1}(\cdot)\right)^{\top}$. Notice that the dimension of $m$ function is $q$ which increases with the sample size $n$. Thus, the parameter $(\alpha, g)$ can be solved from unconditional moment equations $\mathbb{E}\left[m\left(V_{i}, \alpha^{\top} X_{i}, g\left(Z_{i}\right)\right)\right]=0$ for $i=1, \cdots, n$.

According to the estimation procedure in Section 2, define $(\widehat{\alpha}, \widehat{\beta})=\underset{\mathbf{a} \in \mathbb{R}^{p}, \mathbf{b} \in \mathbb{R}^{k}}{\operatorname{argmin}}\left\|M_{n}(\mathbf{a}, \mathbf{b})\right\|^{2}$ where $M_{n}(\mathbf{a}, \mathbf{b})=\frac{1}{\sqrt{q}} \frac{1}{n} \sum_{i=1}^{n} m\left(V_{i}, \mathbf{a}^{\top} X_{i}, \mathbf{b}^{\top} \Phi_{k}\left(Z_{i}\right)\right)$. Thus, $\widehat{\alpha}$ and $\widehat{g}(\cdot):=\widehat{\beta}^{\top} \Phi_{k}(\cdot)$ are the estimates of $(\alpha, g(\cdot))$.

Here, we emphasize that since the $m$ function is linear in both $\alpha^{\top} X_{i}$ and $g\left(Z_{i}\right), M_{n}(\mathbf{a}, \mathbf{b})$ actually has a linear relationship with $\mathbf{a}$ and $\mathbf{b}$,

$$
\begin{aligned}
& M_{n}(\mathbf{a}, \mathbf{b})=\frac{1}{\sqrt{q}} \frac{1}{n} \sum_{i=1}^{n}\left(Y_{i}-\mathbf{a}^{\top} X_{i}-\mathbf{b}^{\top} \Phi_{k}\left(Z_{i}\right)\right) \Phi_{q}\left(Z_{i}\right) \\
= & \frac{1}{\sqrt{q}} \frac{1}{n} \sum_{i=1}^{n} Y_{i} \Phi_{q}\left(Z_{i}\right)-\left(\frac{1}{\sqrt{q}} \frac{1}{n} \sum_{i=1}^{n} \Phi_{q}\left(Z_{i}\right) X_{i}^{\top}\right) \mathbf{a}-\left(\frac{1}{\sqrt{q}} \frac{1}{n} \sum_{i=1}^{n} \Phi_{q}\left(Z_{i}\right) \Phi_{k}\left(Z_{i}\right)^{\top}\right) \mathbf{b} .
\end{aligned}
$$

Accordingly, $(\widehat{\alpha}, \widehat{\beta})$ has an explicit expression simply by OLS. This means that in any similar situation the optimization in Section 2 does not need the compact restrictions.

For $n=200,500$ and 1000 , let $k=\left[C_{1} n^{\tau_{1}}\right]$ with $C_{1}=1$ and $\tau_{1}=1 / 4$, and $p=\left[C_{2} n^{\tau_{2}}\right]$ with $C_{2}=1$ and $\tau_{2}=1 / 5$. Also, let $q=p+k+\nu(\nu \geq 0$ specified in the experiments) satisfy Assumption 3.1. The replication number of the experiment is $M=1000$. We shall report for the estimate of the $g$ function the bias (denoted by $B_{g}(n)$ ), standard deviation (denoted by $\left.\pi_{g}(n)\right)$ and RMSE (denoted by $\Pi_{g}(n)$ ), that is,

$$
\begin{aligned}
B_{g}(n) & :=\frac{1}{M n} \sum_{\ell=1}^{M} \sum_{i=1}^{n}\left[g^{\ell}\left(Z_{i}\right)-g^{\ell}\left(Z_{i}\right)\right], \\
\pi_{g}(n) & :=\left(\frac{1}{M n} \sum_{\ell=1}^{M} \sum_{i=1}^{n}\left[\widehat{g}^{\ell}\left(Z_{i}\right)-\bar{g}\left(Z_{i}\right)\right]^{2}\right)^{1 / 2}, \\
\Pi_{g}(n) & :=\left(\frac{1}{M n} \sum_{\ell=1}^{M} \sum_{i=1}^{n}\left[\widehat{g}^{\ell}\left(Z_{i}\right)-g^{\ell}\left(Z_{i}\right)\right]^{2}\right)^{1 / 2},
\end{aligned}
$$

where the superscript $\ell$ indicates the $\ell$-th replication, $\overline{\widehat{g}}(\cdot)=\Phi_{k}(\cdot)^{\top} \overline{\widehat{\beta}}$ is the average of $\widehat{g}^{\ell}(\cdot)$ over Monto Carlo replications $\ell=1, \cdots, M, g^{\ell}\left(Z_{i}\right)$ means the value of $g$ evaluated for the $Z_{i}$ in the $\ell$-th replication.

Regarding of parameter $\alpha$, we report the following quantities, $B_{\alpha}(n):=\|\alpha-\bar{\alpha}\|$ and $M_{\alpha}(n):=\operatorname{median}(\|\alpha-\widehat{\alpha}\|)$, where $\overline{\widehat{\alpha}}$ is the average of $\widehat{\alpha}^{\ell}$ and median $(\cdots)$ is the median of the sequence over Monto Carlo replications. Notice that, due to the divergence of the dimension, it might not make any sense to compare the estimated results for different sample sizes. 
Table 1: Simulation of Example 5.1, $q=p+k+\nu$

\begin{tabular}{|c|c|c|c|c|c|c|c|}
\hline & \multicolumn{3}{|c|}{$\nu=2$} & & \multicolumn{3}{|c|}{$\nu=4$} \\
\hline$n$ & 300 & 600 & 1000 & $n$ & 300 & 600 & 1000 \\
\hline$B_{g}(n)$ & 0.0046 & -0.0040 & -0.0026 & $B_{g}(n)$ & -0.0023 & -0.0019 & 0.0006 \\
\hline$\pi_{g}(n)$ & 0.3533 & 0.1965 & 0.1948 & $\pi_{g}(n)$ & 0.1660 & 0.1530 & 0.1520 \\
\hline$\Pi_{g}(n)$ & 0.3401 & 0.1700 & 0.1682 & $\Pi_{g}(n)$ & 0.1356 & 0.1217 & 0.1176 \\
\hline$B_{\alpha}(n)$ & 0.0700 & 0.0410 & 0.0684 & $B_{\alpha}(n)$ & 0.0281 & 0.0271 & 0.0501 \\
\hline$M_{\alpha}(n)$ & 0.0355 & 0.0282 & 0.0665 & $M_{\alpha}(n)$ & 0.0259 & 0.0244 & 0.0319 \\
\hline \multicolumn{4}{|c|}{$\nu=6$} & & \multicolumn{3}{|c|}{$\nu=8$} \\
\hline$n$ & 300 & 600 & 1000 & $n$ & 300 & 600 & 1000 \\
\hline$B_{g}(n)$ & 0.0023 & 0.0019 & -0.0000 & $B_{g}(n)$ & 0.0009 & 0.0011 & -0.0000 \\
\hline$\pi_{g}(n)$ & 0.1544 & 0.1445 & 0.1444 & $\pi_{g}(n)$ & 0.1482 & 0.1370 & 0.1359 \\
\hline$\Pi_{g}(n)$ & 0.1218 & 0.1092 & 0.1031 & $\Pi_{g}(n)$ & 0.1176 & 0.1015 & 0.0945 \\
\hline$B_{\alpha}(n)$ & 0.0124 & 0.0267 & 0.0265 & $B_{\alpha}(n)$ & 0.0078 & 0.0048 & 0.0250 \\
\hline$M_{\alpha}(n)$ & 0.0254 & 0.0154 & 0.0464 & $M_{\alpha}(n)$ & 0.0117 & 0.0098 & 0.0306 \\
\hline
\end{tabular}

It can be seen that all of the statistical quantities about the estimator of $g$ are reasonably attenuated with the increase of both the sample size and $\nu$ that provides more information for the parameters to be estimated. For the quantities about the estimator of $\alpha$, we observe that they normally do not decrease with the sample size. This is because, as mentioned before, the dimension of $\alpha$ is increasing with the sample size; and hence it does not make sense to compare them among different sample sizes. However, we find that both quantities decrease with the increase of $\nu$ that gives more moment restrictions.

This is understandable. Because the conditional moment $\mathbb{E}\left[Y_{i}-\alpha^{\top} X_{i}-g\left(Z_{i}\right) \mid Z_{i}\right]$ determines a function $U(z):=\mathbb{E}\left[Y_{i}-\alpha^{\top} X_{i}-g\left(Z_{i}\right) \mid Z_{i}=z\right]$ and $\left\{\varphi_{j}(z)\right\}$ is an orthornomal sequence in the space that contains $U(z)$, the greater the $\nu$ is, the more axes in the space we use to explain the unknown function $U(z)$.

Example 5.2. This experiment uses the model in Example 1.3 in Section 1. Let the distribution function $F(u)=\exp (u) /[1+\exp (u)]$. $Y_{i}$ assumes either 0 or 1 , and

$$
P\left(Y_{i}=1 \mid X_{i}, Z_{i}\right)=F\left(\alpha^{\top} X_{i}+g\left(Z_{i}\right)\right)
$$


for $i=1, \cdots, n$, where $\alpha, X_{i} \in \mathbb{R}^{p}$ and $Z_{i} \in \mathbb{R}$. Here, let $X_{i} \sim N\left(0, \Sigma_{x}\right)$, where $\Sigma_{x}=\left(\sigma_{i, j}\right)_{p \times p}$ with $\sigma_{i, i}=1, \sigma_{i, j}=0.5$ for $|i-j|=1$ and $\sigma_{i, j}=0$ for $|i-j|>1$, and $Z_{i} \sim N(0,1)$. In this experiment, put $\alpha=(0.5,0.3,0, \cdots, 0)^{\top} \in \mathbb{R}^{p}$ and $g(z)=z^{2}+\sin (z)$. The Hilbert space that contains $g(\cdot)$ is $L^{2}\left(\mathbb{R}, \exp \left(-z^{2}\right)\right)$. Let $\left\{p_{j}(z), j \geq 0\right\}$ be the sequence of Hermite polynomials that forms an orthonormal basis in $L^{2}\left(\mathbb{R}, \exp \left(-z^{2}\right)\right)$.

Denote $\Phi_{k}(z)=\left(p_{0}(z), \cdots, p_{k-1}(z)\right)^{\top}$ and similarly to Example 1.3, define

$$
\begin{aligned}
Q_{n}(\alpha, \beta) & :=\ln \prod_{i=1}^{n} F^{Y_{i}}\left(\alpha^{\top} X_{i}+\beta^{\top} \Phi_{k}\left(Z_{i}\right)\right)\left[1-F\left(\alpha^{\top} X_{i}+\beta^{\top} \Phi_{k}\left(Z_{i}\right)\right)\right]^{1-Y_{i}}, \\
M_{n}(\alpha, \beta) & :=\left(\frac{\partial Q_{n}}{\partial \alpha^{\top}}, \frac{\partial Q_{n}}{\partial \beta^{\top}}\right)^{\top}
\end{aligned}
$$

and we have $(\widehat{\alpha}, \widehat{\beta})=\underset{\mathbf{a} \in \mathbb{R}^{p}, \mathbf{b} \in \mathbb{R}^{k}}{\operatorname{argmin}}\left\|M_{n}(\mathbf{a}, \mathbf{b})\right\|^{2}$ and naturally $\widehat{g}(\cdot):=\widehat{\beta}^{\top} \Phi_{k}(\cdot)$ is the estimate of $g(\cdot)$.

For $n=200,500$ and 1000 , let $k=\left[C_{1} n^{\tau_{1}}\right]$ and $p=\left[C_{2} n^{\tau_{2}}\right]$ where $C_{i}$ and $\tau_{i}, i=1,2$, take the same values as in the preceding example. The replication number of the experiment is $M=1000$. We shall report the bias $B_{g}(n)$, standard deviation $\pi_{g}(n)$ and $\operatorname{RMSE} \Pi_{g}(n)$ for the estimator of $g$ and $B_{\alpha}(n)$ and $M_{\alpha}(n)$ for the estimator of $\alpha$ defined in the above example.

Table 2: Simulation results for Example 5.2

\begin{tabular}{lrrrrrrrr}
\hline$n$ & 300 & 600 & 1000 & & $n$ & 300 & 600 & 1000 \\
\cline { 2 - 4 } \cline { 6 - 8 }$B_{\alpha}(n)$ & 0.0130 & 0.0105 & 0.0065 & & $B_{g}(n)$ & -0.0100 & 0.0059 & 0.0037 \\
$M_{\alpha}(n)$ & 0.0125 & 0.0103 & 0.0075 & & $\pi_{g}(n)$ & 0.3608 & 0.3128 & 0.2315 \\
& & & & & $\Pi_{g}(n)$ & 0.3320 & 0.2323 & 0.1732 \\
\hline
\end{tabular}

The moment restriction model is exactly identified, since it is formulated from the partial derivatives that imply $q=p+k$. All results in Table 2 converge satisfactorily, though it seems in this example the estimator of the $g$ function converge a bit slower than the last example. This might be because in the last example there is an explicit solution while this example needs a minimization of the nonlinear distribution function to have the estimators.

\section{Conclusion}

A class of high dimensional semiparametric moment restriction models have been studied using the GMM and sieve method. The consistency and normality of the proposed estimators 
have obtained. A new test statistic has been proposed for over-identification testing and the strong signal can be weakened when our test statistic is used. The theoretical results are verified through finite sample experiments. In addition, we find that the more the number of the moment restrictions, the accurate the estimates, although it may not be interesting to compare the estimates of the high-dimensional parameters for different sample sizes.

\section{Acknowledgement}

We acknowledge Professor Xiaohong Chen for her insightful suggestion and pointing out some relevant references. The first author thanks the financial support from National Natural Science Foundation of China under grant No. 71671143. The second author was supported by the Australian Research Council Discovery Grants Program for its support under Grant numbers: DP150101012 \& DP170104421.

\section{A Lemmas}

Lemma A.1. Under Assumptions 2.1-2.2, 3.1-3.3 we have

1. $\left\|M_{n}(\alpha, \beta)\right\|^{2}=O_{P}\left(\left\|\gamma_{k}(z)\right\|^{2}\right)+O_{P}\left(n^{-1}\right)$.

2. Given $B_{1 n}^{2}+B_{2 n}^{2}=o(n), \sup _{\substack{\|\mathbf{a}\| \leq B_{1 n},\|\mathbf{b}\| \leq B_{2} \\\|(\mathbf{a}-\alpha, \mathbf{b}-\beta)\|>\delta}}\left\|M_{n}(\mathbf{a}, \mathbf{b})\right\|^{-2}=O_{P}(1)$ for each $\delta>0$, when $n$ is large.

Proof. 1. Observe that

$$
\begin{aligned}
\left\|M_{n}(\alpha, \beta)\right\|^{2} & =\left\|\frac{1}{\sqrt{q}} \frac{1}{n} \sum_{i=1}^{n} m\left(V_{i}, \alpha^{\top} X_{i}, \beta^{\top} \Phi_{k}\left(Z_{i}\right)\right)\right\|^{2} \\
& =\frac{1}{q} \sum_{\ell=1}^{q}\left[\frac{1}{n} \sum_{i=1}^{n} m_{\ell}\left(V_{i}, \alpha^{\top} X_{i}, \beta^{\top} \Phi_{k}\left(Z_{i}\right)\right)\right]^{2},
\end{aligned}
$$

where we denote $m(\cdots)=\left(m_{1}(\cdots), \cdots, m_{q}(\cdots)\right)^{\top}$. Moreover,

$$
\begin{aligned}
& \frac{1}{q} \sum_{\ell=1}^{q} \mathbb{E}\left[\frac{1}{n} \sum_{i=1}^{n} m_{\ell}\left(V_{i}, \alpha^{\top} X_{i}, \beta^{\top} \Phi_{k}\left(Z_{i}\right)\right)\right]^{2} \\
= & \frac{1}{q} \sum_{\ell=1}^{q}\left[\frac{1}{n} \sum_{i=1}^{n} \mathbb{E} m_{\ell}\left(V_{i}, \alpha^{\top} X_{i}, \beta^{\top} \Phi_{k}\left(Z_{i}\right)\right)\right]^{2}+\frac{1}{q} \sum_{\ell=1}^{q} \operatorname{Var}\left[\frac{1}{n} \sum_{i=1}^{n} m_{\ell}\left(V_{i}, \alpha^{\top} X_{i}, \beta^{\top} \Phi_{k}\left(Z_{i}\right)\right)\right] \\
= & \frac{1}{q} \sum_{\ell=1}^{q}\left[\mathbb{E} m_{\ell}\left(V_{1}, \alpha^{\top} X_{1}, \beta^{\top} \Phi_{k}\left(Z_{1}\right)\right)\right]^{2}+\frac{1}{q} \frac{1}{n^{2}} \sum_{\ell=1}^{q} \sum_{i=1}^{n} \operatorname{Var}\left[m_{\ell}\left(V_{i}, \alpha^{\top} X_{i}, \beta^{\top} \Phi_{k}\left(Z_{i}\right)\right)\right] \\
= & \frac{1}{q}\left\|\mathbb{E} m\left(V_{1}, \alpha^{\top} X_{1}, \beta^{\top} \Phi_{k}\left(Z_{1}\right)\right)\right\|^{2}+\frac{1}{q} \frac{1}{n} \sum_{\ell=1}^{q} \operatorname{Var}\left(m_{\ell}\left(V_{1}, \alpha^{\top} X_{1}, \beta^{\top} \Phi_{k}\left(Z_{1}\right)\right)\right)
\end{aligned}
$$




$$
\begin{aligned}
& \leq \frac{1}{q}\left\|\mathbb{E} m\left(V_{1}, \alpha^{\top} X_{1}, \beta^{\top} \Phi_{k}\left(Z_{1}\right)\right)\right\|^{2}+\frac{1}{n} \frac{1}{q} \sum_{\ell=1}^{q} \mathbb{E}\left(m_{\ell}^{2}\left(V_{1}, \alpha^{\top} X_{1}, \beta^{\top} \Phi_{k}\left(Z_{1}\right)\right)\right) \\
& =\frac{1}{q}\left\|\mathbb{E} m\left(V_{1}, \alpha^{\top} X_{1}, \beta^{\top} \Phi_{k}\left(Z_{1}\right)\right)\right\|^{2}+\frac{1}{n} \frac{1}{q} \mathbb{E}\left\|m\left(V_{1}, \alpha^{\top} X_{1}, \beta^{\top} \Phi_{k}\left(Z_{1}\right)\right)\right\|^{2}
\end{aligned}
$$

due to the property of the i.i.d. sequence.

Since $\mathbb{E}\left[m\left(V_{1}, \alpha^{\top} X_{1}, g\left(Z_{1}\right)\right)=0\right.$, it follows from Assumption 3.3 that

$$
\begin{aligned}
& \frac{1}{q}\left\|\mathbb{E} m\left(V_{1}, \alpha^{\top} X_{1}, \beta^{\top} \Phi_{k}\left(Z_{1}\right)\right)\right\|^{2} \\
= & \frac{1}{q}\left\|\mathbb{E}\left[m\left(V_{1}, \alpha^{\top} X_{1}, \beta^{\top} \Phi_{k}\left(Z_{1}\right)\right)-m\left(V_{1}, \alpha^{\top} X_{1}, \beta^{\top} \Phi_{k}\left(Z_{1}\right)+\gamma_{k}\left(Z_{1}\right)\right)\right]\right\|^{2} \\
\leq & \left\{\mathbb{E}\left[A\left(V_{1}, X_{1}, Z_{1}\right)\right]\left|\gamma_{k}\left(Z_{1}\right)\right|^{2} \leq \mathbb{E}\left[A\left(V_{1}, X_{1}, Z_{1}\right)\right]^{2} \mathbb{E}\left|\gamma_{k}\left(Z_{1}\right)\right|^{2}\right. \\
\leq & C\left\|\gamma_{k}(z)\right\|^{2}=o(1),
\end{aligned}
$$

by virtue of Assumption 3.1(b), and for the second term,

$$
\begin{aligned}
& \frac{1}{q} \mathbb{E}\left\|m\left(V_{1}, \alpha^{\top} X_{1}, \beta^{\top} \Phi_{k}\left(Z_{1}\right)\right)\right\|^{2} \\
\leq & 2 \frac{1}{q} \mathbb{E}\left\|m\left(V_{1}, \alpha^{\top} X_{1}, g\left(Z_{1}\right)\right)\right\|^{2}+2 \frac{1}{q} \mathbb{E}\left\|m\left(V_{1}, \alpha^{\top} X_{1}, \beta^{\top} \Phi_{k}\left(Z_{1}\right)\right)-m\left(V_{1}, \alpha^{\top} X_{1}, g\left(Z_{1}\right)\right)\right\|^{2} \\
= & O(1)+\mathbb{E}\left[A^{2}\left(V_{1}, X_{1}, Z_{1}\right)\left|\gamma_{k}\left(Z_{1}\right)\right|^{2}\right]=O(1)
\end{aligned}
$$

by the dominated convergence theorem, implying the second term is $O\left(n^{-1}\right)$.

2. First, note that

$$
\begin{aligned}
& M_{n}(\mathbf{a}, \mathbf{b})-\frac{1}{\sqrt{q}} \mathbb{E} m\left(V_{1}, \mathbf{a}^{\top} X_{1}, \mathbf{b}^{\top} \Phi_{k}\left(Z_{1}\right)\right) \\
= & \frac{1}{\sqrt{q}} \frac{1}{n} \sum_{i=1}^{n}\left[m\left(V_{i}, \mathbf{a}^{\top} X_{i}, \mathbf{b}^{\top} \Phi_{k}\left(Z_{i}\right)\right)-\mathbb{E} m\left(V_{i}, \mathbf{a}^{\top} X_{i}, \mathbf{b}^{\top} \Phi_{k}\left(Z_{i}\right)\right)\right] .
\end{aligned}
$$

It follows from the property of i.i.d. sequence and Assumption 3.3 that

$$
\begin{aligned}
& \mathbb{E}\left\|M_{n}(\mathbf{a}, \mathbf{b})-\frac{1}{\sqrt{q}} \mathbb{E} m\left(V_{1}, \mathbf{a}^{\top} X_{1}, \mathbf{b}^{\top} \Phi_{k}\left(Z_{1}\right)\right)\right\|^{2} \\
= & \frac{1}{n^{2}} \sum_{i=1}^{n} \frac{1}{q} \mathbb{E}\left\|m\left(V_{i}, \mathbf{a}^{\top} X_{i}, \mathbf{b}^{\top} \Phi_{k}\left(Z_{i}\right)\right)-\mathbb{E} m\left(V_{i}, \mathbf{a}^{\top} X_{i}, \mathbf{b}^{\top} \Phi_{k}\left(Z_{i}\right)\right)\right\|^{2} \\
\leq & \frac{1}{n} \frac{1}{q} \mathbb{E}\left\|m\left(V_{1}, \mathbf{a}^{\top} X_{1}, \mathbf{b}^{\top} \Phi_{k}\left(Z_{1}\right)\right)\right\|^{2}=O\left(n^{-1}\left(B_{1 n}^{2}+B_{2 n}^{2}\right)\right),
\end{aligned}
$$

uniformly in $\left(\mathbf{a}, \mathbf{b}^{\top} \Phi_{k}(z)\right) \in \Theta_{n}$ by Assumption 3.3, which implies by the triangle inequality that

$$
\left|\left\|M_{n}(\mathbf{a}, \mathbf{b})\right\|-\frac{1}{\sqrt{q}}\left\|\mathbb{E} m\left(V_{1}, \mathbf{a}^{\top} X_{1}, \mathbf{b}^{\top} \Phi_{k}\left(Z_{1}\right)\right)\right\|\right|
$$




$$
\leq\left\|M_{n}(\mathbf{a}, \mathbf{b})-\frac{1}{\sqrt{q}} \mathbb{E} m\left(V_{1}, \mathbf{a}^{\top} X_{1}, \mathbf{b}^{\top} \Phi_{k}\left(Z_{1}\right)\right)\right\|=O_{P}\left(n^{-1 / 2}\left(B_{1 n}+B_{2 n}\right)\right),
$$

that is, $\left\|M_{n}(\mathbf{a}, \mathbf{b})\right\|=\frac{1}{\sqrt{q}}\left\|\mathbb{E} m\left(V_{1}, \mathbf{a}^{\top} X_{1}, \mathbf{b}^{\top} \Phi_{k}\left(Z_{1}\right)\right)\right\|+O_{P}\left(n^{-1 / 2}\left(B_{1 n}+B_{2 n}\right)\right)$ where the last term is independent of $(\mathbf{a}, \mathbf{b})$. This is equivalent to $\left\|M_{n}(\mathbf{a}, \mathbf{b})\right\|^{2}=\frac{1}{q}\left\|\mathbb{E} m\left(V_{1}, \mathbf{a}^{\top} X_{1}, \mathbf{b}^{\top} \Phi_{k}\left(Z_{1}\right)\right)\right\|^{2}+$ $O_{P}\left(n^{-1}\left(B_{1 n}^{2}+B_{2 n}^{2}\right)\right)$ by basic algebra.

Second, for any $\|\mathbf{b}\|^{2} \leq B_{2 n}$, we have $\mathbf{b}^{\top} \Phi_{k}(z) \in \Theta_{2 n}$. Also, $\left\|\mathbf{b}^{\top} \Phi_{k}(z)-g(z)\right\|^{2}=\| \mathbf{b}-$ $\beta\left\|^{2}+\right\| \gamma_{k}(z) \|^{2}$ by the orthogonality of the basis sequence.

For any $\delta>0$, let $n$ be large (so $k$ large) such that $\delta>\left\|\gamma_{k}(z)\right\|$. Moreover, by Assumption 3.2 , regarding of this $\delta>0$ there exists an $\epsilon>0$ such that

$$
\inf _{\substack{(\mathbf{a}, f) \in \Theta \\\|(\mathbf{a}-\alpha, f-g)\| \geq \delta}} \frac{1}{q}\left\|\mathbb{E} m\left(V_{i}, \mathbf{a}^{\top} X_{i}, f\left(Z_{i}\right)\right)\right\|^{2}>\epsilon .
$$

Notice further that

$$
\begin{aligned}
& \inf _{\substack{\|\mathbf{a}\| \leq B_{1 n},\|\mathbf{b}\| \leq B_{2 n} \\
\|(\mathbf{a}-\alpha, \mathbf{b}-\beta)\| \geq \delta}} \frac{1}{q}\left\|\mathbb{E} m\left(V_{i}, \mathbf{a}^{\top} X_{i}, \mathbf{b}^{\top} \Phi_{k}\left(Z_{i}\right)\right)\right\|^{2} \\
& =\inf _{\substack{\|\mathbf{a}\| \leq B_{1 n},\|\mathbf{b}\| \leq B_{2 n} \\
\|\mathbf{a}-\alpha\|^{2}+\|\mathbf{b}-\beta\|^{2} \geq \delta^{2}}} \frac{1}{q}\left\|\mathbb{E} m\left(V_{i}, \mathbf{a}^{\top} X_{i}, \mathbf{b}^{\top} \Phi_{k}\left(Z_{i}\right)\right)\right\|^{2} \\
& \geq \inf _{\substack{\|\mathbf{a}\| \leq B_{1 n},\|\mathbf{b}\| \leq B_{2 n} \\
\|\mathbf{a}-\alpha\|^{2}+\|\mathbf{b}-\beta\|^{2} \geq \delta^{2}-\left\|\gamma_{k}(z)\right\|^{2}}} \frac{1}{q}\left\|\mathbb{E} m\left(V_{i}, \mathbf{a}^{\top} X_{i}, \mathbf{b}^{\top} \Phi_{k}\left(Z_{i}\right)\right)\right\|^{2} \\
& \geq \inf _{\substack{\left(\mathbf{a}, \mathbf{b}^{\top} \Phi_{k}(z)\right) \in \Theta_{n} \\
\|\mathbf{a}-\alpha\|^{2}+\left\|\mathbf{b}^{\top} \Phi_{k}(z)-g(z)\right\|^{2} \geq \delta^{2}}} \frac{1}{q}\left\|\mathbb{E} m\left(V_{i}, \mathbf{a}^{\top} X_{i}, \mathbf{b}^{\top} \Phi_{k}\left(Z_{i}\right)\right)\right\|^{2} \\
& \geq \inf _{\substack{(\mathbf{a}, f) \in \Theta \\
\|\mathbf{a}-\alpha\|^{2}+\|f-g\|^{2} \geq \delta^{2}}} \frac{1}{q}\left\|\mathbb{E} m\left(V_{i}, \mathbf{a}^{\top} X_{i}, f\left(Z_{i}\right)\right)\right\|^{2} \\
& \geq \inf _{\substack{(\mathbf{a}, f) \in \Theta \\
\|(\mathbf{a}-\alpha, f-g)\| \geq \delta}} \frac{1}{q}\left\|\mathbb{E} m\left(V_{i}, \mathbf{a}^{\top} X_{i}, f\left(Z_{i}\right)\right)\right\|^{2}>\epsilon,
\end{aligned}
$$

due to $\Theta_{n} \subset \Theta$, which, along with the approximation in the first part, is tantamount to the assertion.

Denote $m(v, u, w)=\left(m_{1}(v, u, w), \cdots, m_{q}(v, u, w)\right)^{\top}$.

Since $\left\|M_{n}(\mathbf{a}, \mathbf{b})\right\|^{2}=\frac{1}{q n^{2}} \sum_{\ell=1}^{q}\left(\sum_{i=1}^{n} m_{\ell}\left(V_{i}, \mathbf{a}^{\top} X_{i}, \mathbf{b}^{\top} \Phi_{k}\left(Z_{i}\right)\right)\right)^{2}$ we have

$$
\begin{aligned}
\frac{\partial}{\partial \mathbf{a}}\left\|M_{n}(\mathbf{a}, \mathbf{b})\right\|^{2}=2 \frac{1}{q n^{2}} & \sum_{\ell=1}^{q} \sum_{i=1}^{n} m_{\ell}\left(V_{i}, \mathbf{a}^{\top} X_{i}, \mathbf{b}^{\top} \Phi_{k}\left(Z_{i}\right)\right) \\
& \times \sum_{j=1}^{n} \frac{\partial}{\partial u} m_{\ell}\left(V_{j}, \mathbf{a}^{\top} X_{j}, \mathbf{b}^{\top} \Phi_{k}\left(Z_{j}\right)\right) X_{j}
\end{aligned}
$$




$$
\begin{aligned}
\frac{\partial}{\partial \mathbf{b}}\left\|M_{n}(\mathbf{a}, \mathbf{b})\right\|^{2}=2 \frac{1}{q n^{2}} & \sum_{\ell=1}^{q} \sum_{i=1}^{n} m_{\ell}\left(V_{i}, \mathbf{a}^{\top} X_{i}, \mathbf{b}^{\top} \Phi_{k}\left(Z_{i}\right)\right) \\
& \times \sum_{j=1}^{n} \frac{\partial}{\partial w} m_{\ell}\left(V_{j}, \mathbf{a}^{\top} X_{j}, \mathbf{b}^{\top} \Phi_{k}\left(Z_{j}\right)\right) \Phi_{k}\left(Z_{j}\right),
\end{aligned}
$$

and

$$
\begin{aligned}
& \frac{\partial^{2}}{\partial \mathbf{a} \partial \mathbf{a}^{\top}}\left\|M_{n}(\mathbf{a}, \mathbf{b})\right\|^{2}=2 \frac{1}{q n^{2}} \sum_{\ell=1}^{q} \sum_{i=1}^{n} \sum_{j=1}^{n} \frac{\partial}{\partial u} m_{\ell}\left(V_{i}, \mathbf{a}^{\top} X_{i}, \mathbf{b}^{\top} \Phi_{k}\left(Z_{i}\right)\right) \\
& \times \frac{\partial}{\partial u} m_{\ell}\left(V_{j}, \mathbf{a}^{\top} X_{j}, \mathbf{b}^{\top} \Phi_{k}\left(Z_{j}\right)\right) X_{j} X_{i}^{\top} \\
& +2 \frac{1}{q n^{2}} \sum_{\ell=1}^{q} \sum_{i=1}^{n} \sum_{j=1}^{n} m_{\ell}\left(V_{i}, \mathbf{a}^{\top} X_{i}, \mathbf{b}^{\top} \Phi_{k}\left(Z_{i}\right)\right) \\
& \times \frac{\partial^{2}}{\partial u^{2}} m_{\ell}\left(V_{j}, \mathbf{a}^{\top} X_{j}, \mathbf{b}^{\top} \Phi_{k}\left(Z_{j}\right)\right) X_{j} X_{j}^{\top} \\
& \frac{\partial^{2}}{\partial \mathbf{a} \partial \mathbf{b}^{\boldsymbol{\top}}}\left\|M_{n}(\mathbf{a}, \mathbf{b})\right\|^{2}=2 \frac{1}{q n^{2}} \sum_{\ell=1}^{q} \sum_{i=1}^{n} \sum_{j=1}^{n} \frac{\partial}{\partial w} m_{\ell}\left(V_{i}, \mathbf{a}^{\boldsymbol{\top}} X_{i}, \mathbf{b}^{\boldsymbol{\top}} \Phi_{k}\left(Z_{i}\right)\right) \\
& \times \frac{\partial}{\partial u} m_{\ell}\left(V_{j}, \mathbf{a}^{\top} X_{j}, \mathbf{b}^{\top} \Phi_{k}\left(Z_{j}\right)\right) X_{j} \Phi_{k}\left(Z_{i}\right)^{\top} \\
& +2 \frac{1}{q n^{2}} \sum_{\ell=1}^{q} \sum_{i=1}^{n} \sum_{j=1}^{n} m_{\ell}\left(V_{i}, \mathbf{a}^{\top} X_{i}, \mathbf{b}^{\top} \Phi_{k}\left(Z_{i}\right)\right) \\
& \times \frac{\partial^{2}}{\partial u \partial w} m_{\ell}\left(V_{j}, \mathbf{a}^{\top} X_{j}, \mathbf{b}^{\top} \Phi_{k}\left(Z_{j}\right)\right) X_{j} \Phi_{k}\left(Z_{j}\right)^{\top} \\
& \frac{\partial^{2}}{\partial \mathbf{b} \partial \mathbf{b}^{\top}}\left\|M_{n}(\mathbf{a}, \mathbf{b})\right\|^{2}=2 \frac{1}{q n^{2}} \sum_{\ell=1}^{q} \sum_{i=1}^{n} \sum_{j=1}^{n} \frac{\partial}{\partial w} m_{\ell}\left(V_{i}, \mathbf{a}^{\top} X_{i}, \mathbf{b}^{\top} \Phi_{k}\left(Z_{i}\right)\right) \\
& \times \frac{\partial}{\partial w} m_{\ell}\left(V_{j}, \mathbf{a}^{\top} X_{j}, \mathbf{b}^{\top} \Phi_{k}\left(Z_{j}\right)\right) \Phi_{k}\left(Z_{j}\right) \Phi_{k}\left(Z_{i}\right)^{\top} \\
& +2 \frac{1}{q n^{2}} \sum_{\ell=1}^{q} \sum_{i=1}^{n} \sum_{j=1}^{n} m_{\ell}\left(V_{i}, \mathbf{a}^{\top} X_{i}, \mathbf{b}^{\top} \Phi_{k}\left(Z_{i}\right)\right) \\
& \times \frac{\partial^{2}}{\partial w^{2}} m_{\ell}\left(V_{j}, \mathbf{a}^{\top} X_{j}, \mathbf{b}^{\top} \Phi_{k}\left(Z_{j}\right)\right) \Phi_{k}\left(Z_{j}\right) \Phi_{k}\left(Z_{j}\right)^{\top} .
\end{aligned}
$$

The unimportant constant shall be ignored in what follows.

Denote each block of $H_{n}(\mathbf{a}, \mathbf{b})$ by

$$
\begin{aligned}
H_{11}(\mathbf{a}, \mathbf{b}):=\frac{\partial^{2}}{\partial \mathbf{a} \partial \mathbf{a}^{\top}}\left\|M_{n}(\mathbf{a}, \mathbf{b})\right\|^{2}, & H_{12}(\mathbf{a}, \mathbf{b}):=\frac{\partial^{2}}{\partial \mathbf{a} \partial \mathbf{b}^{\top}}\left\|M_{n}(\mathbf{a}, \mathbf{b})\right\|^{2} \\
H_{22}(\mathbf{a}, \mathbf{b}):=\frac{\partial^{2}}{\partial \mathbf{b} \partial \mathbf{b}^{\top}}\left\|M_{n}(\mathbf{a}, \mathbf{b})\right\|^{2} & H_{21}(\mathbf{a}, \mathbf{b})=H_{12}(\mathbf{a}, \mathbf{b})^{\top}
\end{aligned}
$$

and define

$$
h_{11}(\alpha, g):=\frac{1}{q} \sum_{\ell=1}^{q}\left(\mathbb{E} \frac{\partial}{\partial u} m_{\ell}\left(V_{1}, \alpha^{\top} X_{1}, g\left(Z_{1}\right)\right) X_{1}\right)\left(\mathbb{E} \frac{\partial}{\partial u} m_{\ell}\left(V_{1}, \alpha^{\top} X_{1}, g\left(Z_{1}\right)\right) X_{1}\right)^{\top},
$$




$$
\begin{aligned}
& =\frac{1}{q}\left[\mathbb{E}\left(\frac{\partial}{\partial u} m\left(V_{1}, \alpha^{\top} X_{1}, g\left(Z_{1}\right)\right)\right)^{\top} \otimes X_{1}\right]\left[\mathbb{E}\left(\frac{\partial}{\partial u} m\left(V_{1}, \alpha^{\top} X_{1}, g\left(Z_{1}\right)\right)\right)^{\top} \otimes X_{1}\right]^{\top} \\
h_{12}(\alpha, g): & =\frac{1}{q} \sum_{\ell=1}^{q}\left(\mathbb{E} \frac{\partial}{\partial u} m_{\ell}\left(V_{1}, \alpha^{\top} X_{1}, g\left(Z_{1}\right)\right) X_{1}\right)\left(\mathbb{E} \frac{\partial}{\partial w} m_{\ell}\left(V_{1}, \alpha^{\top} X_{1}, g\left(Z_{1}\right)\right) \Phi_{k}\left(Z_{1}\right)\right)^{\top}, \\
& =\frac{1}{q}\left[\mathbb{E}\left(\frac{\partial}{\partial u} m\left(V_{1}, \alpha^{\top} X_{1}, g\left(Z_{1}\right)\right)\right)^{\top} \otimes X_{1}\right]\left[\mathbb{E}\left(\frac{\partial}{\partial w} m\left(V_{1}, \alpha^{\top} X_{1}, g\left(Z_{1}\right)\right)\right)^{\top} \otimes \Phi_{k}\left(Z_{1}\right)\right]^{\top} \\
h_{21}(\alpha, g): & =h_{12}(\alpha, g)^{\top}, \\
h_{22}(\alpha, g): & =\frac{1}{q} \sum_{\ell=1}^{q}\left(\mathbb{E} \frac{\partial}{\partial w} m_{\ell}\left(V_{1}, \alpha^{\top} X_{1}, g\left(Z_{1}\right)\right) \Phi_{k}\left(Z_{1}\right)\right)\left(\mathbb{E} \frac{\partial}{\partial w} m_{\ell}\left(V_{1}, \alpha^{\top} X_{1}, g\left(Z_{1}\right)\right) \Phi_{k}\left(Z_{1}\right)\right)^{\top} \\
& =\frac{1}{q}\left[\mathbb{E}\left(\frac{\partial}{\partial w} m\left(V_{1}, \alpha^{\top} X_{1}, g\left(Z_{1}\right)\right)\right)^{\top} \otimes \Phi_{k}\left(Z_{1}\right)\right]\left[\mathbb{E}\left(\frac{\partial}{\partial w} m\left(V_{1}, \alpha^{\top} X_{1}, g\left(Z_{1}\right)\right)\right)^{\top} \otimes \Phi_{k}\left(Z_{1}\right)\right]^{\top} .
\end{aligned}
$$

Denote

$$
h_{n}(\alpha, g)=\left(\begin{array}{ll}
h_{11}(\alpha, g) & h_{12}(\alpha, g) \\
h_{21}(\alpha, g) & h_{22}(\alpha, g)
\end{array}\right)=\frac{1}{q} \Psi_{n} \Psi_{n}^{\top}
$$

where

$$
\Psi_{n}=\mathbb{E}\left(\begin{array}{c}
\frac{\partial}{\partial u} m\left(V_{1}, \alpha^{\top} X_{1}, g\left(Z_{1}\right)\right)^{\top} \otimes X_{1} \\
\frac{\partial}{\partial w} m\left(V_{1}, \alpha^{\top} X_{1}, g\left(Z_{1}\right)\right)^{\top} \otimes \Phi_{k}\left(Z_{1}\right)
\end{array}\right)_{(p+k) \times q}
$$

Assumption A.1. When sample size is $n$, suppose that

1. $[(\mathrm{i})]$

2. $\mathbb{E}\left\|m\left(V_{1}, \alpha^{\top} X_{1}, g\left(Z_{1}\right)\right)\right\|^{2}=O(q), E\left\|X_{1}\right\|^{2}=O(p)$ and $E\left\|\Phi_{k}\left(Z_{1}\right)\right\|^{2}=O(k)$;

3. $\mathbb{E}\left\|\frac{\partial}{\partial u} m\left(V_{1}, \alpha^{\top} X_{1}, g\left(Z_{1}\right)\right)\right\|^{2}=O(q), \quad$ and $\mathbb{E}\left\|\frac{\partial}{\partial w} m\left(V_{1}, \alpha^{\top} X_{1}, g\left(Z_{1}\right)\right)\right\|^{2}=O(q)$;

4. $\mathbb{E}\left\|\frac{\partial}{\partial u} m\left(V_{1}, \alpha^{\top} X_{1}, g\left(Z_{1}\right)\right) \otimes X_{1}\right\|^{2}=O(p q), \quad$ and

$$
\mathbb{E}\left\|\frac{\partial}{\partial w} m\left(V_{1}, \alpha^{\top} X_{1}, g\left(Z_{1}\right)\right) \otimes \Phi_{k}\left(Z_{1}\right)\right\|^{2}=O(k q) ;
$$

5. $\mathbb{E}\left\|\frac{\partial^{2}}{\partial u^{2}} m\left(V_{1}, \alpha^{\top} X_{1}, g\left(Z_{1}\right)\right) \otimes X_{1} X_{1}^{\top}\right\|^{2}=O\left(p^{2} q\right), \quad$ and

$$
\mathbb{E}\left\|\frac{\partial^{2}}{\partial w^{2}} m\left(V_{1}, \alpha^{\top} X_{1}, g\left(Z_{1}\right)\right) \otimes \Phi_{k}\left(Z_{1}\right) \Phi_{k}\left(Z_{1}\right)^{\top}\right\|^{2}=O\left(k^{2} q\right) .
$$

We have the following comments on the assumption. It is nature to alow that each element of $m$ function has the same second moment that suffices the first supposition in A.1(i). Because the dimension $p$ of $X_{1}$ diverges with $n$, in A.1(i) we allow the second moment $E\left\|X_{1}\right\|^{2}$ diverges too, but, as can be seen in the proof of the following lemma, $E\left\|X_{1}\right\|^{2}=O(p)$ may be 
substituted with some appropriate increasing function of $p$; moreover, $E\left\|\Phi_{k}\left(Z_{1}\right)\right\|^{2}=O(k)$ can be true for many orthogonal sequences given the relation between the densities of $Z_{1}$ and the $L^{2}$ space. In A.1(ii) we impose similar condition for the norm of the function's first partial derivatives. A.1(iii) and (iv) stipulate moment conditions for the norms of the tensor product for regressor and the partial derivatives (the first and second, respectively) of the $m$ function.

\section{Assumption A.2.}

1. $[(i)]$

2. $\left\|\gamma_{k}(z)\right\|^{2} p^{2}=o(1), \quad n^{-1} p^{2}=o(1)$;

3. $\left\|\gamma_{k}(z)\right\|^{2} k^{2}=o(1), \quad n^{-1} k^{2}=o(1)$.

Assumption A.2 stipulates the relations among truncation parameter $k$, the diverging dimension $p$ of the regressor and the sample size. Normally, $\left\|\gamma_{k}(z)\right\|^{2}=O\left(k^{-\tau}\right)$ where $\tau$ is related with the smoothness order of the function $g$. See, for example, Newey [21]. Thus, the assumption implicitly puts some conditions on the smoothness. Notice that the combination of A.2(i) and (ii) implies $\left\|\gamma_{k}(z)\right\|^{2} p k=o(1)$ and $n^{-1} p k=o(1)$ which are used in the proof of the following lemma.

Assumption A.3 The partial derivatives of $m(v, u, w)$ satisfy

1. $[(\mathrm{i})]$

2. $q^{-1 / 2}\left\|\frac{\partial}{\partial u} m\left(V, \mathbf{a}_{1}^{\top} X, f_{1}(Z)\right)-\frac{\partial}{\partial u} m\left(V, \mathbf{a}_{2}^{\top} X, f_{2}(Z)\right)\right\| \leq A_{1}(V, X, Z)\left[\left\|\mathbf{a}_{1}-\mathbf{a}_{2}\right\|+\mid f_{1}(Z)-\right.$ $\left.f_{2}(Z) \mid\right]$ where $\mathbb{E}\left[A_{1}(V, X, Z)^{2}\right]<\infty$ and $\mathbb{E}\left[A_{1}(V, X, Z)^{2}\|X\|^{2}\right]=O(p)$.

3. $q^{-1 / 2}\left\|\frac{\partial}{\partial w} m\left(V, \mathbf{a}_{1}^{\top} X, f_{1}(Z)\right)-\frac{\partial}{\partial w} m\left(V, \mathbf{a}_{2}^{\top} X, f_{2}(Z)\right)\right\| \leq A_{2}(V, X, Z)\left[\left\|\mathbf{a}_{1}-\mathbf{a}_{2}\right\|+\mid f_{1}(Z)-\right.$ $\left.f_{2}(Z) \mid\right]$ where $\mathbb{E}\left[A_{2}(V, X, Z)^{2}\right]<\infty$ and $\mathbb{E}\left[A_{2}(V, X, Z)^{2}\left\|\Phi_{k}(Z)\right\|^{2}\right]=O(k)$.

The assumption is similar to Assumption 3.3 but stipulated for the partial derivatives with additional requirements that $\mathbb{E}\left[A_{1}(V, X, Z)^{2}\|X\|^{2}\right]=O(p)$ and $\mathbb{E}\left[A_{2}(V, X, Z)^{2}\left\|\Phi_{k}(Z)\right\|^{2}\right]=$ $O(k)$. This is the consequence of the partial derivatives and is reasonably diverging with the related dimensions.

Lemma A.2. Under Assumptions 2.1-2.2 and A.1-A.3, (1) $H_{n}(\alpha, \beta)$ is asymptotically almost surely positive definite; (2) let $h_{n}(\alpha, g)$ be defined in (A.1), and we then have $\| H_{n}(\alpha, \beta)-$ $h_{n}(\alpha, g) \|=o_{P}(1)$ as $n \rightarrow \infty$. 
Proof of Lemma A.2. (1) Split the matrix $H_{n}(\alpha, \beta):=\tilde{H}_{n}(\alpha, \beta)+\Delta_{n}(\alpha, \beta)$ where $\tilde{H}_{n}(\alpha, \beta)$ is a symmetric 2-by-2 block matrix with blocks

$$
\begin{aligned}
\tilde{H}_{11}(\alpha, \beta)=\frac{1}{q} \sum_{\ell=1}^{q} & \left(\frac{1}{n} \sum_{j=1}^{n} \frac{\partial}{\partial u} m_{\ell}\left(V_{j}, \alpha^{\top} X_{j}, \beta^{\top} \Phi_{k}\left(Z_{j}\right)\right) X_{j}\right) \\
& \times\left(\frac{1}{n} \sum_{i=1}^{n} \frac{\partial}{\partial u} m_{\ell}\left(V_{i}, \alpha^{\top} X_{i}, \beta^{\top} \Phi_{k}\left(Z_{i}\right)\right) X_{i}\right)^{\top}, \\
\tilde{H}_{12}(\alpha, \beta)=\frac{1}{q} \sum_{\ell=1}^{q} & \left(\frac{1}{n} \sum_{j=1}^{n} \frac{\partial}{\partial u} m_{\ell}\left(V_{j}, \alpha^{\top} X_{j}, \beta^{\top} \Phi_{k}\left(Z_{j}\right)\right) X_{j}\right) \\
& \times\left(\frac{1}{n} \sum_{i=1}^{n} \frac{\partial}{\partial w} m_{\ell}\left(V_{i}, \alpha^{\top} X_{i}, \beta^{\top} \Phi_{k}\left(Z_{i}\right)\right) \Phi_{k}\left(Z_{i}\right)\right)^{\top}, \\
\tilde{H}_{22}(\alpha, \beta)=\frac{1}{q} \sum_{\ell=1}^{q} & \left(\frac{1}{n} \sum_{j=1}^{n} \frac{\partial}{\partial w} m_{\ell}\left(V_{j}, \mathbf{a}^{\top} X_{j}, \mathbf{b}^{\top} \Phi_{k}\left(Z_{j}\right)\right) \Phi_{k}\left(Z_{j}\right)\right)^{\top} \\
& \left(\frac{1}{n} \sum_{i=1}^{n} \frac{\partial}{\partial w} m_{\ell}\left(V_{i}, \mathbf{a}^{\top} X_{i}, \mathbf{b}^{\top} \Phi_{k}\left(Z_{i}\right)\right) \Phi_{k}\left(Z_{i}\right)\right)^{\top},
\end{aligned}
$$

and $\tilde{H}_{21}(\alpha, \beta)=\tilde{H}_{12}(\alpha, \beta)^{\top}$, and $\Delta_{n}(\alpha, \beta)$ has blocks

$$
\begin{aligned}
\Delta_{11}(\alpha, \beta)=\frac{1}{q} \sum_{\ell=1}^{q} & \left(\frac{1}{n} \sum_{i=1}^{n} m_{\ell}\left(V_{i}, \alpha^{\top} X_{i}, \beta^{\top} \Phi_{k}\left(Z_{i}\right)\right)\right) \\
& \times\left(\frac{1}{n} \sum_{j=1}^{n} \frac{\partial^{2}}{\partial u^{2}} m_{\ell}\left(V_{j}, \alpha^{\top} X_{j}, \beta^{\top} \Phi_{k}\left(Z_{j}\right)\right) X_{j} X_{j}^{\top}\right), \\
\Delta_{12}(\alpha, \beta)=\frac{1}{q} \sum_{\ell=1}^{q} & \left(\frac{1}{n} \sum_{i=1}^{n} m_{\ell}\left(V_{i}, \alpha^{\top} X_{i}, \beta^{\top} \Phi_{k}\left(Z_{i}\right)\right)\right) \\
& \times\left(\frac{1}{n} \sum_{j=1}^{n} \frac{\partial^{2}}{\partial u \partial w} m_{\ell}\left(V_{j}, \alpha^{\top} X_{j}, \beta^{\top} \Phi_{k}\left(Z_{j}\right)\right) X_{j} \Phi_{k}\left(Z_{j}\right)^{\top}\right) \\
\Delta_{22}(\alpha, \beta)=\frac{1}{q} \sum_{\ell=1}^{q} & \left(\frac{1}{n} \sum_{i=1}^{n} m_{\ell}\left(V_{i}, \alpha^{\top} X_{i}, \beta^{\top} \Phi_{k}\left(Z_{i}\right)\right)\right) \\
& \times\left(\frac{1}{n} \sum_{j=1}^{n} \frac{\partial^{2}}{\partial w^{2}} m_{\ell}\left(V_{j}, \alpha^{\top} X_{j}, \beta^{\top} \Phi_{k}\left(Z_{j}\right)\right) \Phi_{k}\left(Z_{j}\right) \Phi_{k}\left(Z_{j}\right)^{\top}\right),
\end{aligned}
$$

and $\Delta_{21}(\alpha, \beta)=\Delta_{12}(\alpha, \beta)^{\top}$. To fulfill the assertion, we shall show

(i) $\tilde{H}_{n}(\alpha, \beta)$ is almost surely positive definite and

(ii) $\left\|\Delta_{n}(\alpha, \beta)\right\|=o_{P}(1)$.

Firstly, for any vectors $\mathbf{a} \in \mathbb{R}^{p}$ and $\mathbf{b} \in \mathbb{R}^{k}$ where either $\mathbf{a} \neq 0$ or $\mathbf{b} \neq 0$, we have $\left(\mathbf{a}^{\top}, \mathbf{b}^{\top}\right) \tilde{H}_{n}(\alpha, \beta)\left(\mathbf{a}^{\top}, \mathbf{b}^{\top}\right)^{\top}$ 


$$
\begin{aligned}
= & \frac{1}{q} \sum_{\ell=1}^{q}\left(\frac{1}{n} \sum_{j=1}^{n} \frac{\partial}{\partial u} m_{\ell}\left(V_{j}, \alpha^{\top} X_{j}, \beta^{\top} \Phi_{k}\left(Z_{j}\right)\right) \mathbf{a}^{\top} X_{j}\right)^{2} \\
& +2 \frac{1}{q} \sum_{\ell=1}^{q}\left(\frac{1}{n} \sum_{j=1}^{n} \frac{\partial}{\partial u} m_{\ell}\left(V_{j}, \alpha^{\top} X_{j}, \beta^{\top} \Phi_{k}\left(Z_{j}\right)\right) \mathbf{a}^{\top} X_{j}\right) \\
& \times\left(\frac{1}{n} \sum_{i=1}^{n} \frac{\partial}{\partial w} m_{\ell}\left(V_{i}, \alpha^{\top} X_{i}, \beta^{\top} \Phi_{k}\left(Z_{i}\right)\right) \mathbf{b}^{\top} \Phi_{k}\left(Z_{i}\right)\right) \\
& +\frac{1}{q} \sum_{\ell=1}^{q}\left(\frac{1}{n} \sum_{j=1}^{n} \frac{\partial}{\partial w} m_{\ell}\left(V_{j}, \mathbf{a}^{\top} X_{j}, \mathbf{b}^{\top} \Phi_{k}\left(Z_{j}\right)\right) \mathbf{b}^{\top} \Phi_{k}\left(Z_{j}\right)\right)^{2} \\
= & \frac{1}{q} \sum_{\ell=1}^{q}\left[\frac{1}{n} \sum_{j=1}^{n}\left(\frac{\partial}{\partial u} m_{\ell}\left(V_{j}, \alpha^{\top} X_{j}, \beta^{\top} \Phi_{k}\left(Z_{j}\right)\right) \mathbf{a}^{\top} X_{j}+\frac{\partial}{\partial w} m_{\ell}\left(V_{j}, \mathbf{a}^{\top} X_{j}, \mathbf{b}^{\top} \Phi_{k}\left(Z_{j}\right)\right) \mathbf{b}^{\top} \Phi_{k}\left(Z_{j}\right)\right)\right]^{2},
\end{aligned}
$$

which is almost surely positive. Hence, $\tilde{H}_{n}(\alpha, \beta)$ is almost surely positive definite.

Secondly, to show $\left\|\Delta_{n}(\alpha, \beta)\right\|=o_{P}(1)$, it suffices to prove the result for each block. Indeed, appealing to the triangle inequality and Cauchy-Schwarz inequality,

$$
\begin{aligned}
\left\|\Delta_{11}(\alpha, \beta)\right\|^{2} \leq & \frac{1}{q} \sum_{\ell=1}^{q}\left(\frac{1}{n} \sum_{i=1}^{n} m_{\ell}\left(V_{i}, \alpha^{\top} X_{i}, \beta^{\top} \Phi_{k}\left(Z_{i}\right)\right)\right)^{2} \\
& \times \frac{1}{q} \sum_{\ell=1}^{q}\left\|\frac{1}{n} \sum_{j=1}^{n} \frac{\partial^{2}}{\partial u^{2}} m_{\ell}\left(V_{j}, \alpha^{\top} X_{j}, \beta^{\top} \Phi_{k}\left(Z_{j}\right)\right) X_{j} X_{j}^{\top}\right\|^{2} \\
= & \left\|M_{n}(\alpha, \beta)\right\|^{2} \frac{1}{q} \sum_{\ell=1}^{q}\left\|\frac{1}{n} \sum_{j=1}^{n} \frac{\partial^{2}}{\partial u^{2}} m_{\ell}\left(V_{j}, \alpha^{\top} X_{j}, \beta^{\top} \Phi_{k}\left(Z_{j}\right)\right) X_{j} X_{j}^{\top}\right\|^{2} .
\end{aligned}
$$

Because $\left\|M_{n}(\alpha, \beta)\right\|^{2}=O_{P}\left(\left\|\gamma_{k}(z)\right\|^{2}\right)+O_{P}\left(n^{-1}\right)$ by Lemma A.1, we only need to deal with the second factor. Note that

$$
\begin{gathered}
\frac{1}{q} \sum_{\ell=1}^{q}\left\|\frac{1}{n} \sum_{j=1}^{n} \frac{\partial^{2}}{\partial u^{2}} m_{\ell}\left(V_{j}, \alpha^{\top} X_{j}, \beta^{\top} \Phi_{k}\left(Z_{j}\right)\right) X_{j} X_{j}^{\top}\right\|^{2} \\
\leq \frac{2}{q} \sum_{\ell=1}^{q}\left\|\mathbb{E} \frac{\partial^{2}}{\partial u^{2}} m_{\ell}\left(V_{1}, \alpha^{\top} X_{1}, \beta^{\top} \Phi_{k}\left(Z_{1}\right)\right) X_{1} X_{1}^{\top}\right\|^{2} \\
+\frac{2}{q} \sum_{\ell=1}^{q} \| \frac{1}{n} \sum_{j=1}^{n}\left(\frac{\partial^{2}}{\partial u^{2}} m_{\ell}\left(V_{j}, \alpha^{\top} X_{j}, \beta^{\top} \Phi_{k}\left(Z_{j}\right)\right) X_{j} X_{j}^{\top}\right. \\
\left.-\mathbb{E} \frac{\partial^{2}}{\partial u^{2}} m_{\ell}\left(V_{j}, \alpha^{\top} X_{j}, \beta^{\top} \Phi_{k}\left(Z_{j}\right)\right) X_{j} X_{j}^{\top}\right) \|^{2}
\end{gathered}
$$

where by Assumption A.1 the first term is $O\left(p^{2}\right)$, while by the iid property for the second we have

$$
\frac{1}{q} \sum_{\ell=1}^{q} \mathbb{E} \| \frac{1}{n} \sum_{j=1}^{n}\left(\frac{\partial^{2}}{\partial u^{2}} m_{\ell}\left(V_{j}, \alpha^{\top} X_{j}, \beta^{\top} \Phi_{k}\left(Z_{j}\right)\right) X_{j} X_{j}^{\top}\right.
$$




$$
\begin{aligned}
& \left.-\mathbb{E} \frac{\partial^{2}}{\partial u^{2}} m_{\ell}\left(V_{j}, \alpha^{\top} X_{j}, \beta^{\top} \Phi_{k}\left(Z_{j}\right)\right) X_{j} X_{j}^{\top}\right) \|^{2} \\
= & \frac{1}{n^{2}} \frac{1}{q} \sum_{\ell=1}^{q} \sum_{j=1}^{n} \mathbb{E}\left\|\frac{\partial^{2}}{\partial u^{2}} m_{\ell}\left(V_{j}, \alpha^{\top} X_{j}, \beta^{\top} \Phi_{k}\left(Z_{j}\right)\right) X_{j} X_{j}^{\top}-\mathbb{E} \frac{\partial^{2}}{\partial u^{2}} m_{\ell}\left(V_{j}, \alpha^{\top} X_{j}, \beta^{\top} \Phi_{k}\left(Z_{j}\right)\right) X_{j} X_{j}^{\top}\right\|^{2} \\
= & \frac{1}{n} \frac{1}{q} \sum_{\ell=1}^{q} \mathbb{E}\left\|\frac{\partial^{2}}{\partial u^{2}} m_{\ell}\left(V_{1}, \alpha^{\top} X_{1}, \beta^{\top} \Phi_{k}\left(Z_{1}\right)\right) X_{1} X_{1}^{\top}-\mathbb{E} \frac{\partial^{2}}{\partial u^{2}} m_{\ell}\left(V_{1}, \alpha^{\top} X_{1}, \beta^{\top} \Phi_{k}\left(Z_{1}\right)\right) X_{1} X_{1}^{\top}\right\|^{2} \\
\leq & \frac{1}{n} \frac{1}{q} \sum_{\ell=1}^{q} \mathbb{E}\left\|\frac{\partial^{2}}{\partial u^{2}} m_{\ell}\left(V_{1}, \alpha^{\top} X_{1}, \beta^{\top} \Phi_{k}\left(Z_{1}\right)\right) X_{1} X_{1}^{\top}\right\|^{2} \\
= & \frac{1}{n} \frac{1}{q} \mathbb{E}\left\|\frac{\partial^{2}}{\partial u^{2}} m\left(V_{1}, \alpha^{\top} X_{1}, \beta^{\top} \Phi_{k}\left(Z_{1}\right)\right) \otimes X_{1} X_{1}^{\top}\right\|^{2} \\
= & O\left(n^{-1} p^{2}\right),
\end{aligned}
$$

by Assumption A.1, from which $\left\|\Delta_{11}(\alpha, \beta)\right\|^{2}=O_{P}\left(\left\|\gamma_{k}(z)\right\|^{2} p^{2}\right)+O_{P}\left(n^{-1} p^{2}\right)=o_{P}(1)$.

Similarly,

$$
\left\|\Delta_{12}(\alpha, \beta)\right\|^{2} \leq\left\|M_{n}(\alpha, \beta)\right\|^{2} \frac{1}{q} \sum_{\ell=1}^{q}\left\|\frac{1}{n} \sum_{j=1}^{n} \frac{\partial^{2}}{\partial u \partial w} m_{\ell}\left(V_{j}, \alpha^{\top} X_{j}, \beta^{\top} \Phi_{k}\left(Z_{j}\right)\right) X_{j} \Phi_{k}\left(Z_{j}\right)\right\|^{2}
$$

and for the second factor using again the iid property, we have

$$
\begin{aligned}
& \frac{1}{q} \sum_{\ell=1}^{q} \mathbb{E}\left\|\frac{1}{n} \sum_{j=1}^{n} \frac{\partial^{2}}{\partial u \partial w} m_{\ell}\left(V_{j}, \alpha^{\top} X_{j}, \beta^{\top} \Phi_{k}\left(Z_{j}\right)\right) X_{j} \Phi_{k}\left(Z_{j}\right)^{\top}\right\|^{2} \\
\leq & 2 \frac{1}{q} \sum_{\ell=1}^{q}\left\|\mathbb{E} \frac{\partial^{2}}{\partial u \partial w} m_{\ell}\left(V_{1}, \alpha^{\top} X_{1}, \beta^{\top} \Phi_{k}\left(Z_{1}\right)\right) X_{1} \Phi_{k}\left(Z_{1}\right)^{\top}\right\|^{2} \\
& +2 \frac{1}{n} \frac{1}{q} \sum_{\ell=1}^{q} \mathbb{E} \| \frac{\partial^{2}}{\partial u \partial w} m_{\ell}\left(V_{1}, \alpha^{\top} X_{1}, \beta^{\top} \Phi_{k}\left(Z_{1}\right)\right) X_{1} \Phi_{k}\left(Z_{1}\right)^{\top} \\
\leq & 2 \frac{1}{q} \sum_{\ell=1}^{q}\left\|\mathbb{E} \frac{\partial^{2}}{\partial u \partial w} m_{\ell}\left(V_{1}, \alpha^{\top} X_{1}, \beta^{\top} \Phi_{k}\left(Z_{1}\right)\right) X_{1} \Phi_{k}\left(Z_{1}\right)^{\top}\right\|^{2} \\
& +2 \frac{1}{n} \frac{1}{q} \sum_{\ell=1}^{q} \mathbb{E}\left\|\frac{\partial^{2}}{\partial u \partial w} m_{\ell}\left(V_{1}, \alpha^{\top} X_{1}, \beta^{\top} \Phi_{k}\left(Z_{1}\right)\right) X_{1} \Phi_{k}\left(Z_{1}\right)^{\top}\right\|^{2} \\
= & 2 \frac{1}{q}\left\|\mathbb{E} \frac{\partial^{2}}{\partial u \partial w} m\left(V_{1}, \alpha^{\top} X_{1}, \beta^{\top} \Phi_{k}\left(Z_{1}\right)\right) \otimes X_{1} \Phi_{k}\left(Z_{1}\right)^{\top}\right\|^{2} \\
& \left.+2 \frac{1}{n} \frac{1}{q} \mathbb{E}\left\|\frac{\partial^{2}}{\partial u \partial w} m\left(V_{1}, \alpha^{\top} X_{1}, \beta^{\top} \Phi_{k}\left(Z_{1}\right)\right) \otimes X_{1} \Phi_{k}\left(Z_{1}\right)^{\top}\right\|^{\top} \Phi_{k}\left(Z_{1}\right)\right) X_{1} \Phi_{k}\left(Z_{1}\right)^{\top} \|^{2} \\
= & O(p k)+O\left(n^{-1} p k\right),
\end{aligned}
$$

which implies $\left\|\Delta_{12}(\alpha, \beta)\right\|^{2}=O_{P}\left(\left\|\gamma_{k}(z)\right\|^{2} p k\right)+O_{P}\left(n^{-1} p k\right)=o_{P}(1)$. 
Furthermore,

$$
\left\|\Delta_{22}(\alpha, \beta)\right\|^{2} \leq\left\|M_{n}(\alpha, \beta)\right\|^{2} \frac{1}{q} \sum_{\ell=1}^{q}\left\|\frac{1}{n} \sum_{j=1}^{n} \frac{\partial^{2}}{\partial w^{2}} m_{\ell}\left(V_{j}, \alpha^{\top} X_{j}, \beta^{\top} \Phi_{k}\left(Z_{j}\right)\right) \Phi_{k}\left(Z_{j}\right) \Phi_{k}\left(Z_{j}\right)^{\top}\right\|^{2}
$$

where the second factor can be derived similarly

$$
\begin{aligned}
& \frac{1}{q} \sum_{\ell=1}^{q} \mathbb{E}\left\|\frac{1}{n} \sum_{j=1}^{n} \frac{\partial^{2}}{\partial w^{2}} m_{\ell}\left(V_{j}, \alpha^{\top} X_{j}, \beta^{\top} \Phi_{k}\left(Z_{j}\right)\right) \Phi_{k}\left(Z_{j}\right) \Phi_{k}\left(Z_{j}\right)^{\top}\right\|^{2} \\
\leq & 2 \frac{1}{q}\left\|\mathbb{E} \frac{\partial^{2}}{\partial w^{2}} m\left(V_{1}, \alpha^{\top} X_{1}, \beta^{\top} \Phi_{k}\left(Z_{1}\right)\right) \otimes \Phi_{k}\left(Z_{1}\right) \Phi_{k}\left(Z_{1}\right)^{\top}\right\|^{2} \\
& +2 \frac{1}{n} \frac{1}{q} \mathbb{E}\left\|\frac{\partial^{2}}{\partial w^{2}} m\left(V_{1}, \alpha^{\top} X_{1}, \beta^{\top} \Phi_{k}\left(Z_{1}\right)\right) \otimes \Phi_{k}\left(Z_{1}\right) \Phi_{k}\left(Z_{1}\right)^{\top}\right\|^{2} \\
= & O\left(k^{2}\right)+O\left(n^{-1} k^{2}\right),
\end{aligned}
$$

giving that $\left\|\Delta_{22}(\alpha, \beta)\right\|^{2}=O_{P}\left(\left\|\gamma_{k}(z)\right\|^{2} k^{2}\right)+O_{P}\left(n^{-1} k^{2}\right)=o_{P}(1)$. This finishes the assertion (i).

Now, we show (ii). Because $\left\|H_{n}(\alpha, \beta)-h_{n}(\alpha, g)\right\| \leq\left\|\Delta_{n}(\alpha, \beta)\right\|+\left\|\tilde{H}_{n}(\alpha, \beta)-h_{n}(\alpha, g)\right\|=$ $o_{P}(1)+\left\|\tilde{H}_{n}(\alpha, \beta)-h_{n}(\alpha, g)\right\|$, what we need to show is $\left\|\tilde{H}_{n}(\alpha, \beta)-h_{n}(\alpha, g)\right\|=o_{P}(1)$. It is sufficient to show the result in block-sense. Indeed,

$$
\begin{aligned}
& \tilde{H}_{11}(\alpha, \beta)-h_{11}(\alpha, g) \\
= & \frac{1}{q} \sum_{\ell=1}^{q}\left(\frac{1}{n} \sum_{j=1}^{n} \frac{\partial}{\partial u} m_{\ell}\left(V_{j}, \alpha^{\top} X_{j}, \beta^{\top} \Phi_{k}\left(Z_{j}\right)\right) X_{j}\right)\left(\frac{1}{n} \sum_{i=1}^{n} \frac{\partial}{\partial u} m_{\ell}\left(V_{i}, \alpha^{\top} X_{i}, \beta^{\top} \Phi_{k}\left(Z_{i}\right)\right) X_{i}\right)^{\top} \\
& -\frac{1}{q} \sum_{\ell=1}^{q}\left(\mathbb{E} \frac{\partial}{\partial u} m_{\ell}\left(V_{1}, \alpha^{\top} X_{1}, g\left(Z_{1}\right)\right) X_{1}\right)\left(\mathbb{E} \frac{\partial}{\partial u} m_{\ell}\left(V_{1}, \alpha^{\top} X_{1}, g\left(Z_{1}\right)\right) X_{1}\right)^{\top} \\
= & \frac{1}{q} \sum_{\ell=1}^{q} \frac{1}{n} \sum_{j=1}^{n}\left(\frac{\partial}{\partial u} m_{\ell}\left(V_{j}, \alpha^{\top} X_{j}, \beta^{\top} \Phi_{k}\left(Z_{j}\right)\right) X_{j}-\mathbb{E} \frac{\partial}{\partial u} m_{\ell}\left(V_{j}, \alpha^{\top} X_{j}, g\left(Z_{j}\right)\right) X_{j}\right) \\
& \times\left(\frac{1}{n} \sum_{i=1}^{n} \frac{\partial}{\partial u} m_{\ell}\left(V_{i}, \alpha^{\top} X_{i}, \beta^{\top} \Phi_{k}\left(Z_{i}\right)\right) X_{i}\right)^{\top} \\
+ & \frac{1}{q} \sum_{\ell=1}^{q}\left(\mathbb{E} \frac{\partial}{\partial u} m_{\ell}\left(V_{1}, \alpha^{\top} X_{1}, g\left(Z_{1}\right)\right) X_{1}\right) \\
& \times \frac{1}{n} \sum_{i=1}^{n}\left(\frac{\partial}{\partial u} m_{\ell}\left(V_{i}, \alpha^{\top} X_{i}, \beta^{\top} \Phi_{k}\left(Z_{i}\right)\right) X_{i}-\mathbb{E} \frac{\partial}{\partial u} m_{\ell}\left(V_{i}, \alpha^{\top} X_{i}, g\left(Z_{i}\right)\right) X_{i}\right)^{\top}
\end{aligned}
$$

$:=I_{1}+I_{2}, \quad$ say.

Notice further that

$$
I_{1}=\frac{1}{q} \sum_{\ell=1}^{q} \frac{1}{n} \sum_{j=1}^{n}\left(\frac{\partial}{\partial u} m_{\ell}\left(V_{j}, \alpha^{\top} X_{j}, \beta^{\top} \Phi_{k}\left(Z_{j}\right)\right) X_{j}-\frac{\partial}{\partial u} m_{\ell}\left(V_{j}, \alpha^{\top} X_{j}, g\left(Z_{j}\right)\right) X_{j}\right)
$$




$$
\begin{aligned}
& \times\left(\frac{1}{n} \sum_{i=1}^{n} \frac{\partial}{\partial u} m_{\ell}\left(V_{i}, \alpha^{\top} X_{i}, \beta^{\top} \Phi_{k}\left(Z_{i}\right)\right) X_{i}\right)^{\top} \\
&+\frac{1}{q} \sum_{\ell=1}^{q} \frac{1}{n} \sum_{j=1}^{n}\left(\frac{\partial}{\partial u} m_{\ell}\left(V_{j}, \alpha^{\top} X_{j}, g\left(Z_{j}\right)\right) X_{j}-\mathbb{E} \frac{\partial}{\partial u} m_{\ell}\left(V_{j}, \alpha^{\top} X_{j}, g\left(Z_{j}\right)\right) X_{j}\right) \\
& \times\left(\frac{1}{n} \sum_{i=1}^{n} \frac{\partial}{\partial u} m_{\ell}\left(V_{i}, \alpha^{\top} X_{i}, \beta^{\top} \Phi_{k}\left(Z_{i}\right)\right) X_{i}\right)^{\top} .
\end{aligned}
$$

Hence, using Cauchy-Schwarz inequality,

$$
\begin{aligned}
\left\|I_{1}\right\|^{2} \leq & \frac{1}{q} \sum_{\ell=1}^{q} \\
& \left.\times \frac{1}{n} \sum_{j=1}^{n}\left(\frac{\partial}{\partial u} \sum_{\ell=1}^{q}\left\|\frac{1}{n} \sum_{i=1}^{n} \frac{\partial}{\partial u} m_{\ell}\left(V_{i}, \alpha^{\top} X_{i}, \beta^{\top}, \beta^{\top} \Phi_{k}\left(Z_{i}\right)\right) X_{i}\right\|^{2}\right)-\frac{\partial}{\partial u} m_{\ell}\left(V_{j}, \alpha^{\top} X_{j}, g\left(Z_{j}\right)\right)\right) X_{j} \|^{2} \\
+ & \frac{1}{q} \sum_{\ell=1}^{q}\left\|\frac{1}{n} \sum_{j=1}^{n}\left(\frac{\partial}{\partial u} m_{\ell}\left(V_{j}, \alpha^{\top} X_{j}, g\left(Z_{j}\right)\right) X_{j}-\mathbb{E} \frac{\partial}{\partial u} m_{\ell}\left(V_{j}, \alpha^{\top} X_{j}, g\left(Z_{j}\right)\right) X_{j}\right)\right\|^{2} \\
& \times \frac{1}{q} \sum_{\ell=1}^{q}\left\|\frac{1}{n} \sum_{i=1}^{n} \frac{\partial}{\partial u} m_{\ell}\left(V_{i}, \alpha^{\top} X_{i}, \beta^{\top} \Phi_{k}\left(Z_{i}\right)\right) X_{i}\right\|^{2} \\
:= & I_{11} \times I_{13}+I_{12} \times I_{13}, \quad \text { say. }
\end{aligned}
$$

Due to the i.i.d. property and the Law of Large Number (LLN, hereafter), $I_{11}$ has the same order in probability as

$$
\begin{aligned}
& \frac{1}{q} \sum_{\ell=1}^{q}\left\|\mathbb{E}\left(\frac{\partial}{\partial u} m_{\ell}\left(V_{1}, \alpha^{\top} X_{1}, \beta^{\top} \Phi_{k}\left(Z_{1}\right)\right)-\frac{\partial}{\partial u} m_{\ell}\left(V_{1}, \alpha^{\top} X_{1}, g\left(Z_{1}\right)\right)\right) X_{1}\right\|^{2} \\
= & \frac{1}{q}\left\|\mathbb{E}\left(\frac{\partial}{\partial u} m\left(V_{1}, \alpha^{\top} X_{1}, \beta^{\top} \Phi_{k}\left(Z_{1}\right)\right)-\frac{\partial}{\partial u} m\left(V_{1}, \alpha^{\top} X_{1}, g\left(Z_{1}\right)\right)\right) \otimes X_{1}\right\|^{2} \\
\leq & \mathbb{E}\left[A_{1}\left(V_{1}, X_{1}, Z_{1}\right)^{2}\left\|X_{1}\right\|^{2}\right] \mathbb{E}\left[\gamma_{k}\left(Z_{1}\right)^{2}\right]=O\left(\left\|\gamma_{k}(z)\right\|^{2} p\right),
\end{aligned}
$$

while for $I_{12}$, by the iid property,

$$
\begin{aligned}
\mathbb{E}\left[I_{12}\right] & =\frac{1}{n^{2}} \frac{1}{q} \sum_{\ell=1}^{q} \sum_{j=1}^{n} \mathbb{E}\left\|\frac{\partial}{\partial u} m_{\ell}\left(V_{j}, \alpha^{\top} X_{j}, g\left(Z_{j}\right)\right) X_{j}-\mathbb{E} \frac{\partial}{\partial u} m_{\ell}\left(V_{j}, \alpha^{\top} X_{j}, g\left(Z_{j}\right)\right) X_{j}\right\|^{2} \\
& =\frac{1}{n} \frac{1}{q} \sum_{\ell=1}^{q} \mathbb{E}\left\|\frac{\partial}{\partial u} m_{\ell}\left(V_{1}, \alpha^{\top} X_{1}, g\left(Z_{1}\right)\right) X_{1}-\mathbb{E} \frac{\partial}{\partial u} m_{\ell}\left(V_{1}, \alpha^{\top} X_{1}, g\left(Z_{1}\right)\right) X_{1}\right\|^{2} \\
& \leq \frac{1}{n} \frac{1}{q} \sum_{\ell=1}^{q} \mathbb{E}\left\|\frac{\partial}{\partial u} m_{\ell}\left(V_{1}, \alpha^{\top} X_{1}, g\left(Z_{1}\right)\right) X_{1}\right\|^{2} \\
& \leq \frac{1}{n} \frac{1}{q} \mathbb{E}\left\|\frac{\partial}{\partial u} m\left(V_{1}, \alpha^{\top} X_{1}, g\left(Z_{1}\right)\right) \otimes X_{1}\right\|^{2}=O\left(n^{-1} p\right)
\end{aligned}
$$


by Assumption A.1. Moreover, by virtue of the iid property and the LLN, $I_{13}$ has the same order in probability as

$$
\begin{aligned}
& \frac{1}{q} \sum_{\ell=1}^{q}\left\|\mathbb{E} \frac{\partial}{\partial u} m_{\ell}\left(V_{i}, \alpha^{\top} X_{i}, g\left(Z_{i}\right)\right) X_{i}\right\|^{2} \\
& +\frac{1}{q} \sum_{\ell=1}^{q}\left\|\frac{1}{n} \sum_{i=1}^{n}\left[\frac{\partial}{\partial u} m_{\ell}\left(V_{i}, \alpha^{\top} X_{i}, \beta^{\top} \Phi_{k}\left(Z_{i}\right)\right)-\frac{\partial}{\partial u} m_{\ell}\left(V_{i}, \alpha^{\top} X_{i}, g\left(Z_{i}\right)\right)\right] X_{i}\right\|^{2} \\
= & \frac{1}{q}\left\|\mathbb{E} \frac{\partial}{\partial u} m\left(V_{1}, \alpha^{\top} X_{1}, g\left(Z_{1}\right)\right) \otimes X_{1}\right\|^{2} \\
& +\frac{1}{q} \sum_{\ell=1}^{q}\left\|\mathbb{E}\left[\frac{\partial}{\partial u} m_{\ell}\left(V_{1}, \alpha^{\top} X_{1}, \beta^{\top} \Phi_{k}\left(Z_{1}\right)\right)-\frac{\partial}{\partial u} m_{\ell}\left(V_{1}, \alpha^{\top} X_{1}, g\left(Z_{1}\right)\right)\right] X_{1}\right\|^{2} \\
= & O(p)+\frac{1}{q}\left\|\mathbb{E}\left[\frac{\partial}{\partial u} m\left(V_{1}, \alpha^{\top} X_{1}, \beta^{\top} \Phi_{k}\left(Z_{1}\right)\right)-\frac{\partial}{\partial u} m\left(V_{1}, \alpha^{\top} X_{1}, g\left(Z_{1}\right)\right)\right] \otimes X_{1}\right\|^{2} \\
\leq & O(p)+\left(\mathbb{E}\left[A_{1}\left(V_{1}, X_{1}, Z_{1}\right)\left|\gamma_{k}\left(Z_{1}\right)\right|\left\|X_{1}\right\|\right]\right)^{2} \leq O(p)+O\left(\left\|\gamma_{k}(z)\right\|^{2} p\right)
\end{aligned}
$$

due to Assumptions A.1 and A.3, implying that $\left\|I_{1}\right\|^{2}=O_{P}\left(n^{-1} p^{2}\right)+O_{P}\left(\left\|\gamma_{k}(z)\right\|^{2} p^{2}\right)=o_{P}(1)$ by Assumption A.2.

Now, we consider $I_{2}$. Note that

$$
\begin{aligned}
\left\|I_{2}\right\|^{2} \leq & \frac{1}{q} \sum_{\ell=1}^{q} \| \\
& \times \frac{1}{q} \sum_{\ell=1}^{q} \frac{\partial}{\partial u} m_{\ell}\left(V_{1}, \alpha^{\top} X_{1}, g\left(Z_{1}\right)\right) X_{1}\left\|_{i=1}^{n}\left(\frac{\partial}{\partial u} m_{\ell}\left(V_{i}, \alpha^{\top} X_{i}, \beta^{\top} \Phi_{k}\left(Z_{i}\right)\right) X_{i}-\mathbb{E} \frac{\partial}{\partial u} m_{\ell}\left(V_{i}, \alpha^{\top} X_{i}, g\left(Z_{i}\right)\right) X_{i}\right)\right\|^{2} \\
\leq & 2 \frac{1}{q} \| \\
& \times \frac{\mathrm{E}}{\partial u} m\left(V_{1}, \alpha^{\top} X_{1}, g\left(Z_{1}\right)\right) \otimes X_{1} \|^{2} \\
& \left.+2 \frac{1}{q}\left\|\frac{1}{n} \sum_{i=1}^{n}\left(\frac{\partial}{\partial u} m\left(V_{i}, \alpha^{\top} X_{i}, \beta^{\top} \Phi_{k}\left(Z_{i}\right)\right)-\frac{\partial}{\partial u} m\left(V_{i}, \alpha^{\top} X_{i}, g\left(Z_{i}\right)\right)\right) \otimes X_{j}\right\|_{1} \|^{2}, g\left(Z_{1}\right)\right) \otimes X_{1} \|^{2} \\
& \times \frac{1}{q} \sum_{\ell=1}^{q}\left\|\frac{1}{n} \sum_{i=1}^{n}\left(\frac{\partial}{\partial u} m_{\ell}\left(V_{i}, \alpha^{\top} X_{i}, g\left(Z_{i}\right)\right) X_{i}-\mathbb{E} \frac{\partial}{\partial u} m_{\ell}\left(V_{i}, \alpha^{\top} X_{i}, g\left(Z_{i}\right)\right) X_{i}\right)\right\|^{2} \\
:= & 2 I_{21}\left(I_{22}+I_{23}\right), \quad \operatorname{say.}
\end{aligned}
$$

By Assumption A.1, $I_{21}=O(p)$. In addition, by the LLN $I_{22}$ has the same order in probability as

$$
\begin{aligned}
& \frac{1}{q}\left\|\mathbb{E}\left(\frac{\partial}{\partial u} m\left(V_{1}, \alpha^{\top} X_{1}, \beta^{\top} \Phi_{k}\left(Z_{1}\right)\right)-\frac{\partial}{\partial u} m\left(V_{1}, \alpha^{\top} X_{1}, g\left(Z_{1}\right)\right)\right) \otimes X_{1}\right\|^{2} \\
\leq & \left(\mathbb{E}\left[A_{1}\left(V_{1}, X_{1}, Z_{1}\right)\left|\gamma_{k}\left(Z_{1}\right)\right|\left\|X_{1}\right\|\right]\right)^{2} \leq O(p)\left\|\gamma_{k}(z)\right\|^{2}
\end{aligned}
$$


using Assumption A.3; meanwhile, by the iid property,

$$
\begin{aligned}
\mathbb{E}\left[I_{23}\right] & =\frac{1}{n^{2}} \frac{1}{q} \sum_{\ell=1}^{q} \sum_{i=1}^{n} \mathbb{E}\left\|\frac{\partial}{\partial u} m_{\ell}\left(V_{i}, \alpha^{\top} X_{i}, g\left(Z_{i}\right)\right) X_{i}-\mathbb{E} \frac{\partial}{\partial u} m_{\ell}\left(V_{i}, \alpha^{\top} X_{i}, g\left(Z_{i}\right)\right) X_{i}\right\|^{2} \\
& =\frac{1}{n} \frac{1}{q} \sum_{\ell=1}^{q} \mathbb{E}\left\|\frac{\partial}{\partial u} m_{\ell}\left(V_{1}, \alpha^{\top} X_{1}, g\left(Z_{1}\right)\right) X_{1}-\mathbb{E} \frac{\partial}{\partial u} m_{\ell}\left(V_{1}, \alpha^{\top} X_{1}, g\left(Z_{1}\right)\right) X_{1}\right\|^{2} \\
& \leq \frac{1}{n} \frac{1}{q} \sum_{\ell=1}^{q} \mathbb{E}\left\|\frac{\partial}{\partial u} m_{\ell}\left(V_{1}, \alpha^{\top} X_{1}, g\left(Z_{1}\right)\right) X_{1}\right\|^{2} \\
& =\frac{1}{n} \frac{1}{q} \mathbb{E}\left\|\frac{\partial}{\partial u} m\left(V_{1}, \alpha^{\top} X_{1}, g\left(Z_{1}\right)\right) \otimes X_{1}\right\|^{2}=O\left(n^{-1} p\right)
\end{aligned}
$$

by Assumption A.1. Hence, $\left\|I_{2}\right\|^{2}=O_{P}\left(n^{-1} p^{2}\right)+O_{P}\left(\left\|\gamma_{k}(z)\right\|^{2} p^{2}\right)=o_{P}(1)$. Hence, $\| \tilde{H}_{11}(\alpha, \beta)-$ $h_{11}(\alpha, \beta) \|^{2}=O_{P}(1)$.

Moreover,

$$
\begin{aligned}
& \tilde{H}_{12}(\alpha, \beta)-h_{12}(\alpha, g) \\
= & \frac{1}{q} \sum_{\ell=1}^{q}\left(\frac{1}{n} \sum_{j=1}^{n} \frac{\partial}{\partial u} m_{\ell}\left(V_{j}, \alpha^{\top} X_{j}, \beta^{\top} \Phi_{k}\left(Z_{j}\right)\right) X_{j}\right)\left(\frac{1}{n} \sum_{i=1}^{n} \frac{\partial}{\partial w} m_{\ell}\left(V_{i}, \alpha^{\top} X_{i}, \beta^{\top} \Phi_{k}\left(Z_{i}\right)\right) \Phi_{k}\left(Z_{i}\right)\right)^{\top} \\
& -\frac{1}{q} \sum_{\ell=1}^{q}\left(\mathbb{E} \frac{\partial}{\partial u} m_{\ell}\left(V_{1}, \alpha^{\top} X_{1}, g\left(Z_{1}\right)\right) X_{1}\right)\left(\mathbb{E} \frac{\partial}{\partial u} m_{\ell}\left(V_{1}, \alpha^{\top} X_{1}, g\left(Z_{1}\right)\right) \Phi_{k}\left(Z_{1}\right)\right)^{\top} \\
= & \frac{1}{q} \sum_{\ell=1}^{q}\left(\frac{1}{n} \sum_{j=1}^{n} \frac{\partial}{\partial u} m_{\ell}\left(V_{j}, \alpha^{\top} X_{j}, \beta^{\top} \Phi_{k}\left(Z_{j}\right)\right) X_{j}-\mathbb{E} \frac{\partial}{\partial u} m_{\ell}\left(V_{1}, \alpha^{\top} X_{1}, g\left(Z_{1}\right)\right) X_{1}\right) \\
& \times\left(\frac{1}{n} \sum_{i=1}^{n} \frac{\partial}{\partial w} m_{\ell}\left(V_{i}, \alpha^{\top} X_{i}, \beta^{\top} \Phi_{k}\left(Z_{i}\right)\right) \Phi_{k}\left(Z_{i}\right)\right)^{\top} \\
& +\frac{1}{q} \sum_{\ell=1}^{q}\left(\mathbb{E} \frac{\partial}{\partial u} m_{\ell}\left(V_{1}, \alpha^{\top} X_{1}, g\left(Z_{1}\right)\right) X_{1}\right) \\
& \times\left(\frac{1}{n} \sum_{i=1}^{n} \frac{\partial}{\partial w} m_{\ell}\left(V_{i}, \alpha^{\top} X_{i}, \beta^{\top} \Phi_{k}\left(Z_{i}\right)\right) \Phi_{k}\left(Z_{i}\right)-\mathbb{E} \frac{\partial}{\partial u} m_{\ell}\left(V_{1}, \alpha^{\top} X_{1}, g\left(Z_{1}\right)\right) \Phi_{k}\left(Z_{1}\right)\right)^{\top} \\
:= & I_{3}+I_{4}, \quad \operatorname{say.}
\end{aligned}
$$

Similar to $I_{1},\left\|I_{3}\right\|^{2}=O_{P}\left(n^{-1} p k\right)+O_{P}\left(\left\|\gamma_{k}(z)\right\|^{2} p k\right)=o_{P}(1)$ by Assumption A.2; and similar to $I_{2}$, we may have $\left\|I_{4}\right\|^{2}=O_{P}\left(n^{-1} p k\right)+O_{P}\left(\left\|\gamma_{k}(z)\right\|^{2} p k\right)=o_{P}(1)$. We then have $\left\|\tilde{H}_{12}(\alpha, \beta)-h_{12}(\alpha, \beta)\right\|^{2}=o_{P}(1)$.

Finally, we derive similarly for $\tilde{H}_{22}(\alpha, \beta)-h_{22}(\alpha, \beta)$,

$$
\begin{aligned}
& \tilde{H}_{22}(\alpha, \beta)-h_{22}(\alpha, g) \\
= & \frac{1}{q} \sum_{\ell=1}^{q}\left(\frac{1}{n} \sum_{j=1}^{n} \frac{\partial}{\partial w} m_{\ell}\left(V_{j}, \alpha^{\top} X_{j}, \beta^{\top} \Phi_{k}\left(Z_{j}\right)\right) \Phi_{k}\left(Z_{j}\right)\right)\left(\frac{1}{n} \sum_{i=1}^{n} \frac{\partial}{\partial w} m_{\ell}\left(V_{i}, \alpha^{\top} X_{i}, \beta^{\top} \Phi_{k}\left(Z_{i}\right)\right) \Phi_{k}\left(Z_{i}\right)\right)^{\top}
\end{aligned}
$$




$$
\begin{aligned}
& -\frac{1}{q} \sum_{\ell=1}^{q}\left(\mathbb{E} \frac{\partial}{\partial w} m_{\ell}\left(V_{1}, \alpha^{\top} X_{1}, g\left(Z_{1}\right)\right) \Phi_{k}\left(Z_{1}\right)\right)\left(\mathbb{E} \frac{\partial}{\partial w} m_{\ell}\left(V_{1}, \alpha^{\top} X_{1}, g\left(Z_{1}\right)\right) \Phi_{k}\left(Z_{1}\right)\right)^{\top} \\
= & \frac{1}{q} \sum_{\ell=1}^{q}\left(\frac{1}{n} \sum_{j=1}^{n} \frac{\partial}{\partial w} m_{\ell}\left(V_{j}, \alpha^{\top} X_{j}, \beta^{\top} \Phi_{k}\left(Z_{j}\right)\right) \Phi_{k}\left(Z_{j}\right)-\mathbb{E} \frac{\partial}{\partial w} m_{\ell}\left(V_{1}, \alpha^{\top} X_{1}, g\left(Z_{1}\right)\right) \Phi_{k}\left(Z_{1}\right)\right) \\
& \times\left(\frac{1}{n} \sum_{i=1}^{n} \frac{\partial}{\partial w} m_{\ell}\left(V_{i}, \alpha^{\top} X_{i}, \beta^{\top} \Phi_{k}\left(Z_{i}\right)\right) \Phi_{k}\left(Z_{i}\right)\right)^{\top} \\
+ & \frac{1}{q} \sum_{\ell=1}^{q}\left(\mathbb{E} \frac{\partial}{\partial w} m_{\ell}\left(V_{1}, \alpha^{\top} X_{1}, g\left(Z_{1}\right)\right) \Phi_{k}\left(Z_{1}\right)\right) \\
& \times\left(\frac{1}{n} \sum_{i=1}^{n} \frac{\partial}{\partial w} m_{\ell}\left(V_{i}, \alpha^{\top} X_{i}, \beta^{\top} \Phi_{k}\left(Z_{i}\right)\right) \Phi_{k}\left(Z_{i}\right)-\mathbb{E} \frac{\partial}{\partial w} m_{\ell}\left(V_{1}, \alpha^{\top} X_{1}, g\right) \Phi_{k}\left(Z_{1}\right)\right)^{\top} \\
:= & I_{5}+I_{6}, \quad \text { say. }
\end{aligned}
$$

Using the same approach we have $\left\|I_{5}\right\|^{2}=O_{P}\left(n^{-1} k^{2}\right)+O_{P}\left(\left\|\gamma_{k}(z)\right\|^{2} k^{2}\right)=o_{P}(1)$ and $\left\|I_{6}\right\|^{2}=O_{P}\left(n^{-1} k^{2}\right)+O_{P}\left(\left\|\gamma_{k}(z)\right\|^{2} k^{2}\right)=o_{P}(1)$ by Assumption A.2. The whole proof is complete.

Denote $S_{n}(\mathbf{a}, \mathbf{b})=\left(S_{1 n}(\mathbf{a}, \mathbf{b})^{\top}, S_{2 n}(\mathbf{a}, \mathbf{b})^{\top}\right)^{\top}$, where

$$
\begin{aligned}
& S_{1 n}(\mathbf{a}, \mathbf{b})=\frac{\partial}{\partial \mathbf{a}}\left\|M_{n}(\mathbf{a}, \mathbf{b})\right\|^{2}=\frac{1}{q n^{2}} \sum_{\ell=1}^{q} \sum_{i=1}^{n} m_{\ell}\left(V_{i}, \mathbf{a}^{\top} X_{i}, \mathbf{b}^{\top} \Phi_{k}\left(Z_{i}\right)\right) \\
& \times \sum_{j=1}^{n} \frac{\partial}{\partial u} m_{\ell}\left(V_{j}, \mathbf{a}^{\top} X_{j}, \mathbf{b}^{\top} \Phi_{k}\left(Z_{j}\right)\right) X_{j} \\
& S_{2 n}(\mathbf{a}, \mathbf{b})=\frac{\partial}{\partial \mathbf{b}}\left\|M_{n}(\mathbf{a}, \mathbf{b})\right\|^{2}=\frac{1}{q n^{2}} \sum_{\ell=1}^{q} \sum_{i=1}^{n} m_{\ell}\left(V_{i}, \mathbf{a}^{\top} X_{i}, \mathbf{b}^{\top} \Phi_{k}\left(Z_{i}\right)\right) \\
& \times \sum_{j=1}^{n} \frac{\partial}{\partial w} m_{\ell}\left(V_{j}, \mathbf{a}^{\top} X_{j}, \mathbf{b}^{\top} \Phi_{k}\left(Z_{j}\right)\right) \Phi_{k}\left(Z_{j}\right) .
\end{aligned}
$$

We now focus on $S_{n}(\alpha, \beta)$ with sub-vectors $S_{1 n}(\alpha, \beta)$ and $S_{2 n}(\alpha, \beta)$. Define

$$
\begin{aligned}
s_{1 n}(\alpha, g) & =\frac{1}{q n} \sum_{\ell=1}^{q} \sum_{i=1}^{n} m_{\ell}\left(V_{i}, \alpha^{\top} X_{i}, g\left(Z_{i}\right)\right) \mathbb{E} \frac{\partial}{\partial u} m_{\ell}\left(V_{1}, \alpha^{\top} X_{1}, g\left(Z_{1}\right)\right) X_{1}, \\
& =\left[\frac{1}{q} \mathbb{E}\left(\frac{\partial}{\partial u} m\left(V_{1}, \alpha^{\top} X_{1}, g\left(Z_{1}\right)\right)^{\top} \otimes X_{1}\right)\right] \frac{1}{n} \sum_{i=1}^{n} m\left(V_{i}, \alpha^{\top} X_{i}, g\left(Z_{i}\right)\right), \\
s_{2 n}(\alpha, g) & =\frac{1}{q n} \sum_{\ell=1}^{q} \sum_{i=1}^{n} m_{\ell}\left(V_{i}, \alpha^{\top} X_{i}, g\left(Z_{i}\right)\right) \mathbb{E} \frac{\partial}{\partial w} m_{\ell}\left(V_{1}, \alpha^{\top} X_{1}, g\left(Z_{1}\right)\right) \Phi_{k}\left(Z_{1}\right) \\
& =\left[\frac{1}{q} \mathbb{E}\left(\frac{\partial}{\partial w} m\left(V_{1}, \alpha^{\top} X_{1}, g\left(Z_{1}\right)\right)^{\top} \otimes \Phi_{k}\left(Z_{1}\right)\right)\right] \frac{1}{n} \sum_{i=1}^{n} m\left(V_{i}, \alpha^{\top} X_{i}, g\left(Z_{i}\right)\right),
\end{aligned}
$$

and hence

$$
s_{n}(\alpha, g)=\left(s_{1 n}(\alpha, g)^{\top}, s_{2 n}(\alpha, g)^{\top}\right)^{\top}=\frac{1}{q} \Psi_{n} \frac{1}{n} \sum_{i=1}^{n} m\left(V_{i}, \alpha^{\top} X_{i}, g\left(Z_{i}\right)\right)
$$


where $\Psi_{n}$ is given by (A.1).

Lemma A.3. Under Assumptions 2.1-2.2, 3.1, 3.3, A.1-A.3, as $n \rightarrow \infty$ we have

$$
\left\|S_{n}(\alpha, \beta)-s_{n}(\alpha, g)\right\|=o_{P}(1)
$$

Proof. It is sufficient to show that $\left\|S_{1 n}(\alpha, \beta)-s_{1 n}(\alpha, g)\right\|=o_{P}(1)$ and $\left\|S_{2 n}(\alpha, \beta)-s_{2 n}(\alpha, g)\right\|=$ $o_{P}(1)$. Observe that

$$
\begin{aligned}
& S_{1 n}(\alpha, \beta)-s_{1 n}(\alpha, g) \\
=\frac{1}{q} \sum_{\ell=1}^{q} & \frac{1}{n} \sum_{i=1}^{n}\left[m_{\ell}\left(V_{i}, \alpha^{\top} X_{i}, \beta^{\top} \Phi_{k}\left(Z_{i}\right)\right)-m_{\ell}\left(V_{i}, \alpha^{\top} X_{i}, g\left(Z_{i}\right)\right)\right] \\
& \times \frac{1}{n} \sum_{j=1}^{n} \frac{\partial}{\partial u} m_{\ell}\left(V_{j}, \alpha^{\top} X_{j}, \beta^{\top} \Phi_{k}\left(Z_{j}\right)\right) X_{j} \\
+ & \frac{1}{q} \sum_{\ell=1}^{q} \frac{1}{n} \sum_{i=1}^{n} m_{\ell}\left(V_{i}, \alpha^{\top} X_{i}, g\left(Z_{i}\right)\right) \\
& \times \frac{1}{n} \sum_{j=1}^{n}\left(\frac{\partial}{\partial u} m_{\ell}\left(V_{j}, \alpha^{\top} X_{j}, \beta^{\top} \Phi_{k}\left(Z_{j}\right)\right)-\frac{\partial}{\partial u} m_{\ell}\left(V_{j}, \alpha^{\top} X_{j}, g\left(Z_{j}\right)\right)\right) X_{j} \\
+ & \frac{1}{q} \sum_{\ell=1}^{q} \frac{1}{n} \sum_{i=1}^{n} m_{\ell}\left(V_{i}, \alpha^{\top} X_{i}, g\left(Z_{i}\right)\right) \\
& \times \frac{1}{n} \sum_{j=1}^{n}\left(\frac{\partial}{\partial u} m_{\ell}\left(V_{j}, \alpha^{\top} X_{j}, g\left(Z_{j}\right)\right) X_{j}-\mathbb{E} \frac{\partial}{\partial u} m_{\ell}\left(V_{j}, \alpha^{\top} X_{j}, g\left(Z_{j}\right)\right) X_{j}\right) \\
:= & I_{1}+I_{2}+I_{3}, \quad \operatorname{say.}
\end{aligned}
$$

Then, using Cauchy-Schwarz inequality gives

$$
\begin{aligned}
&\left\|I_{1}\right\|^{2} \leq \frac{1}{q} \sum_{\ell=1}^{q}\left(\frac{1}{n} \sum_{i=1}^{n}\left[m_{\ell}\left(V_{i}, \alpha^{\top} X_{i}, \beta^{\top} \Phi_{k}\left(Z_{i}\right)\right)-m_{\ell}\left(V_{i}, \alpha^{\top} X_{i}, g\left(Z_{i}\right)\right)\right]\right)^{2} \\
& \times \frac{1}{q} \sum_{\ell=1}^{q}\left\|\frac{1}{n} \sum_{j=1}^{n} \frac{\partial}{\partial u} m_{\ell}\left(V_{j}, \alpha^{\top} X_{j}, \beta^{\top} \Phi_{k}\left(Z_{j}\right)\right) X_{j}\right\|^{2} \\
&:=I_{11} \times I_{12}, \quad \text { say. }
\end{aligned}
$$

Observe further that

$$
\begin{aligned}
\mathbb{E}\left[I_{11}\right]= & \frac{1}{q} \sum_{\ell=1}^{q} \mathbb{E}\left(\frac{1}{n} \sum_{i=1}^{n}\left[m_{\ell}\left(V_{i}, \alpha^{\top} X_{i}, \beta^{\top} \Phi_{k}\left(Z_{i}\right)\right)-m_{\ell}\left(V_{i}, \alpha^{\top} X_{i}, g\left(Z_{i}\right)\right)\right]\right)^{2} \\
= & \frac{1}{q} \sum_{\ell=1}^{q} \operatorname{Var}\left(\frac{1}{n} \sum_{i=1}^{n}\left[m_{\ell}\left(V_{i}, \alpha^{\top} X_{i}, \beta^{\top} \Phi_{k}\left(Z_{i}\right)\right)-m_{\ell}\left(V_{i}, \alpha^{\top} X_{i}, g\left(Z_{i}\right)\right)\right]\right) \\
& +\frac{1}{q} \sum_{\ell=1}^{q}\left(\frac{1}{n} \sum_{i=1}^{n} \mathbb{E}\left[m_{\ell}\left(V_{i}, \alpha^{\top} X_{i}, \beta^{\top} \Phi_{k}\left(Z_{i}\right)\right)-m_{\ell}\left(V_{i}, \alpha^{\top} X_{i}, g\left(Z_{i}\right)\right)\right]\right)^{2}
\end{aligned}
$$




$$
\begin{aligned}
= & \frac{1}{q} \sum_{\ell=1}^{q} \frac{1}{n^{2}} \sum_{i=1}^{n} \operatorname{Var}\left[m_{\ell}\left(V_{i}, \alpha^{\top} X_{i}, \beta^{\top} \Phi_{k}\left(Z_{i}\right)\right)-m_{\ell}\left(V_{i}, \alpha^{\top} X_{i}, g\left(Z_{i}\right)\right)\right] \\
& +\frac{1}{q} \sum_{\ell=1}^{q}\left(\mathbb{E} m_{\ell}\left(V_{1}, \alpha^{\top} X_{1}, \beta^{\top} \Phi_{k}\left(Z_{1}\right)\right)\right)^{2} \\
\leq & \frac{1}{n} \frac{1}{q} \sum_{\ell=1}^{q} \operatorname{Var}\left[m_{\ell}\left(V_{1}, \alpha^{\top} X_{1}, \beta^{\top} \Phi_{k}\left(Z_{1}\right)\right)-m_{\ell}\left(V_{1}, \alpha^{\top} X_{1}, g\left(Z_{1}\right)\right)\right] \\
& +\frac{1}{q}\left\|\mathbb{E} m\left(V_{1}, \alpha^{\top} X_{1}, \beta^{\top} \Phi_{k}\left(Z_{1}\right)\right)\right\|^{2} \\
\leq & \frac{1}{n} \frac{1}{q} \sum_{\ell=1}^{q} \mathbb{E}\left[m_{\ell}\left(V_{1}, \alpha^{\top} X_{1}, \beta^{\top} \Phi_{k}\left(Z_{1}\right)\right)-m_{\ell}\left(V_{1}, \alpha^{\top} X_{1}, g\left(Z_{1}\right)\right)\right]^{2} \\
& +\frac{1}{q}\left\|\mathbb{E} m\left(V_{1}, \alpha^{\top} X_{1}, \beta^{\top} \Phi_{k}\left(Z_{1}\right)\right)\right\|^{2} \\
\leq & \frac{1}{n} \frac{1}{q} \mathbb{E}\left\|m\left(V_{1}, \alpha^{\top} X_{1}, \beta^{\top} \Phi_{k}\left(Z_{1}\right)\right)-m\left(V_{1}, \alpha^{\top} X_{1}, g\left(Z_{1}\right)\right)\right\|^{2} \\
& +\frac{1}{q}\left\|\mathbb{E}\left[m\left(V_{1}, \alpha^{\top} X_{1}, \beta^{\top} \Phi_{k}\left(Z_{1}\right)\right)-m\left(V_{1}, \alpha^{\top} X_{1}, g\left(Z_{1}\right)\right)\right]\right\|^{2} \\
\leq & \left.\frac{1}{n} \mathbb{E}\left|A\left(V_{1}, X_{1}, Z_{1}\right) \gamma_{k}\left(Z_{1}\right)\right|^{2}+\mathbb{E}\left|A\left(V_{1}, X_{1}, Z_{1}\right)\right|^{2}\right)\left\|\gamma_{k}(z)\right\|^{2} \\
= & o\left(n^{-1}\right)+O\left(\left\|\gamma_{k}(z)\right\|^{2}\right)
\end{aligned}
$$

by Assumptions 3.1 and 3.3, the dominated convergence theorem and Cauchy-Schwarz inequality. Moreover, it is clear by Assumptions 3.3 and A.1 that

$$
\mathbb{E}\left[I_{12}\right] \leq \frac{1}{q} \mathbb{E}\left\|\frac{\partial}{\partial u} m\left(V_{1}, \alpha^{\top} X_{1}, g\left(Z_{1}\right)\right) \otimes X_{1}\right\|^{2}=O(p) .
$$

Hence, $I_{1}=o_{P}(1)$ by Assumption A.2.

For $I_{2}$, by Cauchy-Schwarz inequality again,

$$
\begin{aligned}
&\left\|I_{2}\right\|^{2} \leq \frac{1}{q} \sum_{\ell=1}^{q} \\
&\left(\frac{1}{n} \sum_{i=1}^{n} m_{\ell}\left(V_{i}, \alpha^{\top} X_{i}, g\left(Z_{i}\right)\right)\right)^{2} \\
& \times \frac{1}{q} \sum_{\ell=1}^{q}\left\|\frac{1}{n} \sum_{j=1}^{n} \frac{\partial}{\partial u} m_{\ell}\left(V_{j}, \alpha^{\top} X_{j}, \beta^{\top} \Phi_{k}\left(Z_{j}\right)\right) X_{j}-\frac{\partial}{\partial u} m_{\ell}\left(V_{j}, \alpha^{\top} X_{j}, g\left(Z_{j}\right)\right) X_{j}\right\|^{2} \\
&:=I_{21} \times I_{22}, \quad \text { say. }
\end{aligned}
$$

By virtue of the iid property and Assumption A.1,

$$
\begin{aligned}
\mathbb{E}\left[I_{21}\right] & =\frac{1}{n^{2}} \frac{1}{q} \sum_{\ell=1}^{q} \sum_{i=1}^{n} \mathbb{E} m_{\ell}\left(V_{i}, \alpha^{\top} X_{i}, g\left(Z_{i}\right)\right)^{2} \\
& =\frac{1}{n} \frac{1}{q} \sum_{\ell=1}^{q} \mathbb{E} m_{\ell}\left(V_{1}, \alpha^{\top} X_{1}, g\left(Z_{1}\right)\right)^{2}=\frac{1}{n} \frac{1}{q} \mathbb{E}\left\|m\left(V_{1}, \alpha^{\top} X_{1}, g\left(Z_{1}\right)\right)\right\|^{2} \\
& =O\left(n^{-1}\right) .
\end{aligned}
$$


Meanwhile, invoking of the LLN $I_{22}$ has the same order in probability as

$$
\begin{aligned}
& \frac{1}{q} \sum_{\ell=1}^{q}\left\|\mathbb{E}\left[\frac{\partial}{\partial u} m_{\ell}\left(V_{1}, \alpha^{\top} X_{1}, \beta^{\top} \Phi_{k}\left(Z_{1}\right)\right) X_{1}-\frac{\partial}{\partial u} m_{\ell}\left(V_{1}, \alpha^{\top} X_{1}, g\left(Z_{1}\right)\right) X_{1}\right]\right\|^{2} \\
= & \frac{1}{q}\left\|\mathbb{E}\left[\frac{\partial}{\partial u} m\left(V_{1}, \alpha^{\top} X_{1}, \beta^{\top} \Phi_{k}\left(Z_{1}\right)\right) \otimes X_{1}-\frac{\partial}{\partial u} m_{\ell}\left(V_{1}, \alpha^{\top} X_{1}, g\left(Z_{1}\right)\right) \otimes X_{1}\right]\right\|^{2} \\
\leq & \left|\mathbb{E}\left[A_{1}\left(V_{1}, X_{1}, Z_{1}\right)\left|\gamma_{k}\left(Z_{1}\right)\right|\left\|X_{1}\right\|\right]\right|^{2} \leq O\left(\left\|\gamma_{k}(z)\right\|^{2} p\right) \\
= & o(1)
\end{aligned}
$$

due to Assumption A.3 and Cauchy-Schwarz inequality, implying $I_{2}=o_{P}(1)$.

Again, using Cauchy-Schwarz inequality gives

$$
\begin{aligned}
\left\|I_{3}\right\|^{2} \leq & \frac{1}{q} \sum_{\ell=1}^{q} \\
& \left(\frac{1}{n} \sum_{i=1}^{n} m_{\ell}\left(V_{i}, \alpha^{\top} X_{i}, g\left(Z_{i}\right)\right)\right)^{2} \\
& \times \frac{1}{q} \sum_{\ell=1}^{q}\left\|\frac{1}{n} \sum_{j=1}^{n}\left(\frac{\partial}{\partial u} m_{\ell}\left(V_{j}, \alpha^{\top} X_{j}, g\left(Z_{j}\right)\right) X_{j}-\mathbb{E} \frac{\partial}{\partial u} m_{\ell}\left(V_{j}, \alpha^{\top} X_{j}, g\left(Z_{j}\right)\right) X_{j}\right)\right\|^{2} \\
= & O_{P}\left(n^{-1}\right) O_{P}(p)=O_{P}\left(n^{-1} p\right)=o_{P}(1)
\end{aligned}
$$

due to the iid property and Assumption A.1. This finishes the proof of $\left\|S_{1 n}(\alpha, \beta)-s_{1 n}(\alpha, g)\right\|=$ $o_{P}(1)$.

Now, we are to show $\left\|S_{2 n}(\alpha, \beta)-s_{2 n}(\alpha, g)\right\|=o_{P}(1)$. Note that

$$
\begin{aligned}
& S_{2 n}(\alpha, \beta)-s_{2 n}(\alpha, g) \\
= & \frac{1}{q n^{2}} \sum_{\ell=1}^{q} \sum_{i=1}^{n} m_{\ell}\left(V_{i}, \alpha^{\top} X_{i}, \beta^{\top} \Phi_{k}\left(Z_{i}\right)\right) \\
& \times \sum_{j=1}^{n} \frac{\partial}{\partial w} m_{\ell}\left(V_{j}, \alpha^{\top} X_{j}, \beta^{\top} \Phi_{k}\left(Z_{j}\right)\right) \Phi_{k}\left(Z_{j}\right) \\
& -\frac{1}{q n} \sum_{\ell=1}^{q} \sum_{i=1}^{n} m_{\ell}\left(V_{i}, \alpha^{\top} X_{i}, g\left(Z_{i}\right)\right) \mathbb{E} \frac{\partial}{\partial w} m_{\ell}\left(V_{1}, \alpha^{\top} X_{1}, g\left(Z_{1}\right)\right) \Phi_{k}\left(Z_{1}\right) \\
= & \frac{1}{q n^{2}} \sum_{\ell=1}^{q} \sum_{i=1}^{n}\left[m_{\ell}\left(V_{i}, \alpha^{\top} X_{i}, \beta^{\top} \Phi_{k}\left(Z_{i}\right)\right)-m_{\ell}\left(V_{i}, \alpha^{\top} X_{i}, g\left(Z_{i}\right)\right)\right] \\
& \times \sum_{j=1}^{n} \frac{\partial}{\partial w} m_{\ell}\left(V_{j}, \alpha^{\top} X_{j}, \beta^{\top} \Phi_{k}\left(Z_{j}\right)\right) \Phi_{k}\left(Z_{j}\right) \\
& +\frac{1}{q n^{2}} \sum_{\ell=1}^{q} \sum_{i=1}^{n} m_{\ell}\left(V_{i}, \alpha^{\top} X_{i}, g\left(Z_{i}\right)\right) \\
& \times \sum_{j=1}^{n}\left(\frac{\partial}{\partial w} m_{\ell}\left(V_{j}, \alpha^{\top} X_{j}, \beta^{\top} \Phi_{k}\left(Z_{j}\right)\right)-\frac{\partial}{\partial w} m_{\ell}\left(V_{j}, \alpha^{\top} X_{j}, g\left(Z_{j}\right)\right)\right) \Phi_{k}\left(Z_{j}\right) \\
& +\frac{1}{q n} \sum_{\ell=1}^{q} \sum_{i=1}^{n} m_{\ell}\left(V_{i}, \alpha^{\top} X_{i}, g\left(Z_{i}\right)\right)
\end{aligned}
$$




$$
\begin{aligned}
& \quad \times\left(\frac{1}{n} \sum_{j=1}^{n} \frac{\partial}{\partial w} m_{\ell}\left(V_{j}, \alpha^{\top} X_{j}, g\left(Z_{j}\right)\right) \Phi_{k}\left(Z_{j}\right)-\mathbb{E} \frac{\partial}{\partial w} m_{\ell}\left(V_{1}, \alpha^{\top} X_{1}, g\left(Z_{1}\right)\right) \Phi_{k}\left(Z_{1}\right)\right) \\
& :=I_{4}+I_{5}+I_{6}, \quad \text { say. }
\end{aligned}
$$

Note further by Cauchy-Schwarz inequality that

$$
\begin{aligned}
\left\|I_{4}\right\|^{2} \leq & \frac{1}{q} \sum_{\ell=1}^{q}\left(\frac{1}{n} \sum_{i=1}^{n}\left[m_{\ell}\left(V_{i}, \alpha^{\top} X_{i}, \beta^{\top} \Phi_{k}\left(Z_{i}\right)\right)-m_{\ell}\left(V_{i}, \alpha^{\top} X_{i}, g\left(Z_{i}\right)\right)\right]\right)^{2} \\
& \times \frac{1}{q} \sum_{\ell=1}^{q}\left\|\frac{1}{n} \sum_{j=1}^{n} \frac{\partial}{\partial w} m_{\ell}\left(V_{j}, \alpha^{\top} X_{j}, \beta^{\top} \Phi_{k}\left(Z_{j}\right)\right) \Phi_{k}\left(Z_{j}\right)\right\|^{2} \\
\leq 2 \frac{1}{q} \sum_{\ell=1}^{q} & \left(\frac{1}{n} \sum_{i=1}^{n}\left[m_{\ell}\left(V_{i}, \alpha^{\top} X_{i}, \beta^{\top} \Phi_{k}\left(Z_{i}\right)\right)-m_{\ell}\left(V_{i}, \alpha^{\top} X_{i}, g\left(Z_{i}\right)\right)\right]\right)^{2} \\
& \times \frac{1}{q} \sum_{\ell=1}^{q}\left\|\frac{1}{n} \sum_{j=1}^{n}\left[\frac{\partial}{\partial w} m_{\ell}\left(V_{j}, \alpha^{\top} X_{j}, \beta^{\top} \Phi_{k}\left(Z_{j}\right)\right)-\frac{\partial}{\partial w} m_{\ell}\left(V_{j}, \alpha^{\top} X_{j}, g\left(Z_{j}\right)\right)\right] \Phi_{k}\left(Z_{j}\right)\right\|^{2} \\
+ & 2 \frac{1}{q} \sum_{\ell=1}^{q}\left(\frac{1}{n} \sum_{i=1}^{n}\left[m_{\ell}\left(V_{i}, \alpha^{\top} X_{i}, \beta^{\top} \Phi_{k}\left(Z_{i}\right)\right)-m_{\ell}\left(V_{i}, \alpha^{\top} X_{i}, g\left(Z_{i}\right)\right)\right]\right)^{2} \\
& \times \frac{1}{q} \sum_{\ell=1}^{q}\left\|\frac{1}{n} \sum_{j=1}^{n} \frac{\partial}{\partial w} m_{\ell}\left(V_{j}, \alpha^{\top} X_{j}, g\left(Z_{j}\right)\right) \Phi_{k}\left(Z_{j}\right)\right\|^{2},
\end{aligned}
$$

where due to Assumption A.3 the second term is the leading one, which by the LLN has the same order as

$$
\begin{gathered}
\frac{1}{q} \sum_{\ell=1}^{q}\left(\mathbb{E}\left[m_{\ell}\left(V_{1}, \alpha^{\top} X_{1}, \beta^{\top} \Phi_{k}\left(Z_{1}\right)\right)-m_{\ell}\left(V_{1}, \alpha^{\top} X_{1}, g\left(Z_{1}\right)\right)\right]\right)^{2} \\
\times \frac{1}{q} \sum_{\ell=1}^{q}\left\|\mathbb{E} \frac{\partial}{\partial w} m_{\ell}\left(V_{1}, \alpha^{\top} X_{1}, g\left(Z_{1}\right)\right) \Phi_{k}\left(Z_{1}\right)\right\|^{2} \\
=\frac{1}{q}\left\|\mathbb{E}\left[m\left(V_{1}, \alpha^{\top} X_{1}, \beta^{\top} \Phi_{k}\left(Z_{1}\right)\right)-m\left(V_{1}, \alpha^{\top} X_{1}, g\left(Z_{1}\right)\right)\right]\right\|^{2} \\
\times \frac{1}{q}\left\|\mathbb{E} \frac{\partial}{\partial w} m\left(V_{1}, \alpha^{\top} X_{1}, g\left(Z_{1}\right)\right) \otimes \Phi_{k}\left(Z_{1}\right)\right\|^{2} \\
\leq\left|\mathbb{E}\left[A\left(V_{1}, X_{1}, Z_{1}\right) \gamma_{k}\left(Z_{1}\right)\right]\right|^{2} O(k) \leq O\left(\left\|\gamma_{k}(z)\right\|^{2} k\right)=o(1)
\end{gathered}
$$

in probability by Assumption A.2 as $n \rightarrow \infty$.

Moreover, invoking Assumptions A.2-A.3, $I_{5}=o_{P}(1)$. Finally,

$$
\begin{aligned}
\left\|I_{6}\right\|^{2} \leq & \frac{1}{q} \sum_{\ell=1}^{q}\left(\frac{1}{n} \sum_{i=1}^{n} m_{\ell}\left(V_{i}, \alpha^{\top} X_{i}, g\left(Z_{i}\right)\right)\right)^{2} \\
& \times \frac{1}{q} \sum_{\ell=1}^{q}\left\|\frac{1}{n} \sum_{j=1}^{n}\left[\frac{\partial}{\partial w} m_{\ell}\left(V_{j}, \alpha^{\top} X_{j}, g\left(Z_{j}\right)\right) \Phi_{k}\left(Z_{j}\right)-\mathbb{E} \frac{\partial}{\partial w} m_{\ell}\left(V_{i}, \alpha^{\top} X_{i}, g\left(Z_{i}\right)\right) \Phi_{k}\left(Z_{i}\right)\right]\right\|^{2}
\end{aligned}
$$


$:=I_{61} \times I_{62}, \quad$ say

Here, $I_{61}=I_{21}$ and thus $\mathbb{E}\left[I_{61}\right]=O\left(n^{-1}\right)$. Meanwhile,

$$
\begin{aligned}
\mathbb{E}\left[I_{62}\right] & =\frac{1}{q} \frac{1}{n^{2}} \sum_{\ell=1}^{q} \sum_{j=1}^{n} \mathbb{E}\left\|\frac{\partial}{\partial w} m_{\ell}\left(V_{j}, \alpha^{\top} X_{j}, g\left(Z_{j}\right)\right) \Phi_{k}\left(Z_{j}\right)-\mathbb{E} \frac{\partial}{\partial w} m_{\ell}\left(V_{i}, \alpha^{\top} X_{i}, g\left(Z_{i}\right)\right) \Phi_{k}\left(Z_{i}\right)\right\|^{2} \\
& =\frac{1}{q} \frac{1}{n} \sum_{\ell=1}^{q} \mathbb{E}\left\|\frac{\partial}{\partial w} m_{\ell}\left(V_{1}, \alpha^{\top} X_{1}, g\left(Z_{1}\right)\right) \Phi_{k}\left(Z_{1}\right)-\mathbb{E} \frac{\partial}{\partial w} m_{\ell}\left(V_{1}, \alpha^{\top} X_{1}, g\left(Z_{1}\right)\right) \Phi_{k}\left(Z_{1}\right)\right\|^{2} \\
& =\frac{1}{q} \frac{1}{n} \mathbb{E}\left\|\frac{\partial}{\partial w} m\left(V_{1}, \alpha^{\top} X_{1}, g\left(Z_{1}\right)\right) \otimes \Phi_{k}\left(Z_{1}\right)-\mathbb{E} \frac{\partial}{\partial w} m\left(V_{1}, \alpha^{\top} X_{1}, g\left(Z_{1}\right)\right) \otimes \Phi_{k}\left(Z_{1}\right)\right\|^{2} \\
& \leq \frac{1}{q} \frac{1}{n} \mathbb{E}\left\|\frac{\partial}{\partial w} m\left(V_{1}, \alpha^{\top} X_{1}, g\left(Z_{1}\right)\right) \otimes \Phi_{k}\left(Z_{1}\right)\right\|^{2}=O\left(n^{-1} k\right)=o(1)
\end{aligned}
$$

appealing to Assumptions A.1-A.2, implying $\left\|I_{6}\right\|^{2}=o_{P}\left(n^{-1} k\right)=o_{P}(1)$. The proof is complete.

\section{B Proofs of the main results}

Proof of Theorem 3.1. In Lemma A.1, we have shown that

(i) $\left\|M_{n}(\alpha, \beta)\right\|^{2}=o_{P}(1)$,

(ii) $\sup _{\|\mathbf{a}\| \leq B_{1 n},\|\mathbf{b}\| \leq B_{2 n}}\left\|M_{n}(\mathbf{a}, \mathbf{b})\right\|^{-2}=O_{P}(1)$ for each $\delta>0$.

Fix $\epsilon>0$ and $\delta>0$. Assertion (ii) means that there exists a large but fixed $M$ for which

$$
\limsup P\left(\sup _{\substack{\|\mathbf{a}\| \leq B_{1 n},\|\mathbf{b}\| \leq B_{2 n} \\\|(\mathbf{a}-\alpha, \mathbf{b}-\beta)\|>\delta}}\left\|M_{n}(\mathbf{a}, \mathbf{b})\right\|^{-2}>M\right)<\epsilon .
$$

Meanwhile, by the definition of the estimator and (i) we have

$$
\left\|M_{n}(\widehat{\alpha}, \widehat{\beta})\right\|^{2}=\inf _{\|\mathbf{a}\| \leq B_{1 n},\|\mathbf{b}\| \leq B_{2 n}}\left\|M_{n}(\mathbf{a}, \mathbf{b})\right\|^{2} \leq\left\|M_{n}(\alpha, \beta)\right\|^{2}=o_{P}(1)
$$

which gives

$$
P\left(\left\|M_{n}(\widehat{\alpha}, \widehat{\beta})\right\|^{-2}>M\right) \rightarrow 1
$$

It follows that, with probability of at least $1-2 \epsilon$ for all $n$ large enough,

$$
\left\|M_{n}(\widehat{\alpha}, \widehat{\beta})\right\|^{-2}>M \geq \sup _{\substack{\|\mathbf{a}\| \leq B_{1 n},\|\mathbf{b}\| \leq B_{2 n} \\\|(\mathbf{a}-\alpha, \mathbf{b}-\beta)\|>\delta}}\left\|M_{n}(\mathbf{a}, \mathbf{b})\right\|^{-2} .
$$


Hence, the inclusion $(\widehat{\alpha}, \widehat{\beta}) \in\left\{(\mathbf{a}, \mathbf{b}):\|\mathbf{a}\| \leq B_{1 n},\|\mathbf{b}\| \leq B_{2 n},\|(\mathbf{a}-\alpha, \mathbf{b}-\beta)\|>\delta\right\}$ holds with probability at most $2 \epsilon$,

$$
\lim \sup P(\|(\widehat{\alpha}-\alpha, \widehat{\beta}-\beta)\|>\delta) \leq 2 \epsilon .
$$

As $\epsilon$ and $\delta$ are arbitrarily chosen, we have $\|(\widehat{\alpha}-\alpha, \widehat{\beta}-\beta)\| \rightarrow_{P} 0$. Notice further that

$$
\begin{aligned}
\|(\widehat{\alpha}-\alpha, \widehat{g}(z)-g(z))\|^{2} & =\|\widehat{\alpha}-\alpha\|^{2}+\int[\widehat{g}(z)-g(z)]^{2} \pi(z) d z \\
& =\|\widehat{\alpha}-\alpha\|^{2}+\int\left[(\widehat{\beta}-\beta)^{\top} \Phi_{k}(z)-\gamma_{k}(z)\right]^{2} \pi(z) d z \\
& =\|\widehat{\alpha}-\alpha\|^{2}+\|\widehat{\beta}-\beta\|^{2}+\left\|\gamma_{k}(z)\right\|^{2} \\
& =\|(\widehat{\alpha}-\alpha, \widehat{\beta}-\beta)\|^{2}+\left\|\gamma_{k}(z)\right\|^{2} \rightarrow_{P} 0
\end{aligned}
$$

as $n, k \rightarrow \infty$, by the orthogonality of the basis sequence, which then completes the proof.

Proof of Theorem 3.2. By the first order condition $S_{n}(\widehat{\alpha}, \widehat{\beta})=0$, consistency and Taylor expansion, we have expansion

$$
0=S_{n}(\widehat{\alpha}, \widehat{\beta})=S_{n}(\alpha, \beta)+H_{n}(\alpha, \beta)\left(\begin{array}{c}
\widehat{\alpha}-\alpha \\
\widehat{\beta}-\beta
\end{array}\right),
$$

where higher order term is omitted. As shown in Lemmas A.2-A.3, under Assumptions 2.1-2.2, 3.1, 3.3 and A.1-A.3 in Appendix A, $H_{n}(\alpha, \beta)$ is asymptotically positive definite and $H_{n}(\alpha, \beta)$ and $S_{n}(\alpha, \beta)$ are approximated by $h_{n}(\alpha, g)$ and $s_{n}(\alpha, g)$ (defined in (A.1) and (A.2)), respectively, that is, $\left\|H_{n}(\alpha, \beta)-h_{n}(\alpha, g)\right\|=o_{P}(1)$ and $\left\|S_{n}(\alpha, \beta)-s_{n}(\alpha, g)\right\|=o_{P}(1)$. Thence,

$$
\left(\begin{array}{c}
\widehat{\alpha}-\alpha \\
\widehat{\beta}-\beta
\end{array}\right)=-H_{n}(\alpha, \beta)^{-1} S_{n}(\alpha, \beta)=-h_{n}(\alpha, g)^{-1} s_{n}(\alpha, g)\left(1+o_{P}(1)\right)
$$

Noting that $\widehat{g}(z)-g(z)=\Phi_{k}(z)^{\top}(\widehat{\beta}-\beta)-\gamma_{k}(z)$, the linearity of Fréchet derivative and ignoring the higher order term in the definition of Fréchet derivative,

$$
\begin{aligned}
& \left(\begin{array}{c}
\mathscr{L}(\widehat{\alpha})-\mathscr{L}(\alpha) \\
\mathscr{F}(\widehat{g})-\mathscr{F}(g)
\end{array}\right)=\left(\begin{array}{c}
\mathscr{L}(\widehat{\alpha}-\alpha) \\
\mathscr{F}^{\prime}(g)(\widehat{g}(z)-g(z))
\end{array}\right) \\
= & \left(\begin{array}{c}
\mathscr{L}(\widehat{\alpha}-\alpha) \\
\mathscr{F}^{\prime}(g) \Phi_{k}(z)^{\top}(\widehat{\beta}-\beta)
\end{array}\right)-\left(\begin{array}{c}
0 \\
\mathscr{F}^{\prime}(g) \gamma_{k}(z)
\end{array}\right) \\
= & \left(\begin{array}{cc}
\mathscr{L} & 0 \\
0 & \mathscr{F}^{\prime}(g) \Phi_{k}(z)^{\top}
\end{array}\right)\left(\begin{array}{c}
\widehat{\alpha}-\alpha \\
\widehat{\beta}-\beta
\end{array}\right)-\left(\begin{array}{c}
0 \\
\mathscr{F}^{\prime}(g) \gamma_{k}(z)
\end{array}\right)
\end{aligned}
$$




$$
\begin{aligned}
& =-\left(\begin{array}{cc}
\mathscr{L} & 0 \\
0 & \mathscr{F}^{\prime}(g) \Phi_{k}(z)^{\top}
\end{array}\right) h_{n}(\alpha, g)^{-1} s_{n}(\alpha, g)-\left(\begin{array}{c}
0 \\
\mathscr{F}^{\prime}(g) \gamma_{k}(z)
\end{array}\right) \\
& :=\Lambda_{1 n}+\Lambda_{2 n}, \quad \text { say. }
\end{aligned}
$$

Recall $h_{n}(\alpha, g)=\frac{1}{q} \Psi_{n} \Psi_{n}^{\top}$ and $s_{n}(\alpha, g)=\frac{1}{q} \Psi_{n} \frac{1}{n} \sum_{i=1}^{n} m\left(V_{i}, \alpha^{\top} X_{i}, g\left(Z_{i}\right)\right)$ by (A.1) and (A.2). Hence, $\Lambda_{1 n}=\frac{1}{n} \Gamma_{n}\left(\Psi_{n} \Psi_{n}^{\top}\right)^{-1} \Psi_{n} \sum_{i=1}^{n} m\left(V_{i}, \alpha^{\top} X_{i}, g\left(Z_{i}\right)\right)$ where

$$
\Gamma_{n}=-\left(\begin{array}{cc}
\mathscr{L} & 0 \\
0 & \mathscr{F}^{\prime}(g(z)) \Phi_{k}(z)^{\top}
\end{array}\right)
$$

Then, the covariance matrix of $\sqrt{n} \Lambda_{1 n}$ is

$$
\Sigma_{n}^{2}:=\Gamma_{n}\left(\Psi_{n} \Psi_{n}^{\top}\right)^{-1} \Psi_{n} \Xi_{n} \Psi_{n}^{\top}\left(\Psi_{n} \Psi_{n}^{\top}\right)^{-1} \Gamma_{n}^{\top}
$$

in which $\Xi_{n}:=\mathbb{E}\left[m\left(V_{1}, \alpha^{\top} X_{1}, g\left(Z_{1}\right)\right) m\left(V_{1}, \alpha^{\top} X_{1}, g\left(Z_{1}\right)\right)^{\top}\right]$. It follows from the standard central limit theorem that $\sqrt{n} \Sigma_{n}^{-1} \Lambda_{1 n} \rightarrow_{D} N\left(0, I_{r+s}\right)$ as $n \rightarrow \infty$. Then the assertion follows because of $\sqrt{n} \Sigma_{n}^{-1}\left(\mathbf{0}_{r}^{\top}, \mathscr{F}^{\prime}(g) \gamma_{k}(z)^{\top}\right)^{\top}=o(1)$, yielding $\sqrt{n} \Lambda_{2 n}=o(1)$.

Proof of Proposition 3.1. The assertions (1) and (2) can be shown similarly to Lemmas 3.4 and 3.5 in Pakes and Pollard [24]. For brevity we omit the proof. For (3), factor $\Xi_{n}=C_{n} C_{n}^{\top}$ and denote $\Omega_{n}=\left[\Psi_{n} W \Psi_{n}^{\top}\right]^{-1} \Psi_{n} W C_{n}$ and $T_{n}=\Omega_{n}-\left[\Psi_{n} \Xi_{n}^{-1} \Psi_{n}^{\top}\right]^{-1} \Psi_{n}\left(C_{n}^{-1}\right)^{\top}$. It follows that

$$
T_{n} T_{n}^{\top}=\Omega_{n} \Omega_{n}^{\top}-\left[\Psi_{n} \Xi_{n}^{-1} \Psi_{n}^{\top}\right]^{-1}
$$

from which

$$
\Gamma_{n}\left[\Psi_{n} W \Psi_{n}^{\top}\right]^{-1} \Psi_{n} W \Xi_{n} W \Psi_{n}^{\top}\left[\Psi_{n} W \Psi_{n}^{\top}\right]^{-1} \Gamma_{n}^{\top} \geq \Gamma_{n}\left[\Psi_{n} \Xi_{n}^{-1} \Psi_{n}^{\top}\right]^{-1} \Gamma_{n}^{\top}
$$

for all $W$ satisfying the conditions, in view of the nonnegative definiteness of $T_{n} T_{n}^{\top}$.

Proof of Theorem 4.1. By the conventional central limit theorem

$$
\left(\sum_{i=1}^{n}\left[\kappa^{\top} m\left(V_{i}, \alpha^{\top} X_{i}, g\left(Z_{i}\right)\right)\right]^{2}\right)^{-1 / 2} \sum_{i=1}^{n} \kappa^{\top} m\left(V_{i}, \alpha^{\top} X_{i}, g\left(Z_{i}\right)\right) \rightarrow_{D} N(0,1),
$$

as $n \rightarrow \infty$ for any $\kappa \in \mathbb{R}^{q}$ such that $\|\kappa\|=1$.

Thus, the result follows immediately if we show

$$
L_{n}(\widehat{\alpha}, \widehat{\beta} ; \kappa)=\left(\sum_{i=1}^{n}\left[\kappa^{\top} m\left(V_{i}, \alpha^{\top} X_{i}, g\left(Z_{i}\right)\right)\right]^{2}\right)^{-1 / 2} \sum_{i=1}^{n} \kappa^{\top} m\left(V_{i}, \alpha^{\top} X_{i}, g\left(Z_{i}\right)\right)+o_{P}(1) .
$$

Toward this end, we shall show

$$
\text { (1). } \frac{1}{n} D_{n}(\widehat{\alpha}, \widehat{\beta} ; \kappa)^{2}-\frac{1}{n} \sum_{i=1}^{n}\left[\kappa^{\top} m\left(V_{i}, \alpha^{\top} X_{i}, g\left(Z_{i}\right)\right)\right]^{2}=o_{P}(1) ; \text { and }
$$


(2). $\frac{1}{\sqrt{n}} \sum_{i=1}^{n} \kappa^{\top} m\left(V_{i}, \widehat{\alpha}^{\top} X_{i}, \widehat{\beta}^{\top} \Phi_{k}\left(Z_{i}\right)\right)-\frac{1}{\sqrt{n}} \sum_{i=1}^{n} \kappa^{\top} m\left(V_{i}, \alpha^{\top} X_{i}, g\left(Z_{i}\right)\right)=o_{P}(1)$.

(1). Notice that

$$
\begin{aligned}
\frac{1}{n} D_{n}(\widehat{\alpha}, \widehat{\beta} ; \kappa)^{2}= & \frac{1}{n} \sum_{i=1}^{n}\left[\kappa^{\top} m\left(V_{i}, \widehat{\alpha}^{\top} X_{i}, \widehat{\beta}^{\top} \Phi_{k}\left(Z_{i}\right)\right)\right]^{2} \\
= & \frac{1}{n} \sum_{i=1}^{n}\left[\kappa^{\top} m\left(V_{i}, \alpha^{\top} X_{i}, g\left(Z_{i}\right)\right)\right]^{2} \\
& +\frac{1}{n} \sum_{i=1}^{n}\left\{\left[\kappa^{\top} m\left(V_{i}, \widehat{\alpha}^{\top} X_{i}, \widehat{g}\left(Z_{i}\right)\right)\right]^{2}-\left[\kappa^{\top} m\left(V_{i}, \alpha^{\top} X_{i}, g\left(Z_{i}\right)\right)\right]^{2}\right\}
\end{aligned}
$$

and we shall show that the second term is $o_{P}(1)$. First of all, we need the convergence rate of $\|\widehat{\alpha}-\alpha\|^{2}$ and $\|\widehat{\beta}-\beta\|^{2}$. It follows from (B.1) in the proof of Theorem 3.2 that $\left((\widehat{\alpha}-\alpha)^{\top},(\widehat{\beta}-\beta)^{\top}\right)$ has leading term $h_{n}(\alpha, g)^{-1} s_{n}(\alpha, g)$. Then, by the expressions of $h_{n}(\alpha, g)$ and $s_{n}(\alpha, g)$ it is readily seen that $\|\widehat{\alpha}-\alpha\|^{2}=O_{P}(q / n)$ and $\|\widehat{\beta}-\beta\|^{2}=O_{P}(q / n)$.

Moreover, by the first order Taylor expansion,

$$
\begin{aligned}
& \frac{1}{n} \sum_{i=1}^{n}\left|\left[\kappa^{\top} m\left(V_{i}, \widehat{\alpha}^{\top} X_{i}, \widehat{g}\left(Z_{i}\right)\right)\right]^{2}-\left[\kappa^{\top} m\left(V_{i}, \alpha^{\top} X_{i}, g\left(Z_{i}\right)\right)\right]^{2}\right| \\
& \leq \frac{1}{n} \sum_{i=1}^{n}\left|\kappa^{\top}\left[m\left(V_{i}, \widehat{\alpha}^{\top} X_{i}, \widehat{g}\left(Z_{i}\right)\right)-m\left(V_{i}, \alpha^{\top} X_{i}, g\left(Z_{i}\right)\right)\right]\right|^{2} \\
& +2 \frac{1}{n} \sum_{i=1}^{n}\left|\kappa^{\top}\left[m\left(V_{i}, \widehat{\alpha}^{\top} X_{i}, \widehat{g}\left(Z_{i}\right)\right)-m\left(V_{i}, \alpha^{\top} X_{i}, g\left(Z_{i}\right)\right)\right]\right|\left|\kappa^{\top} m\left(V_{i}, \alpha^{\top} X_{i}, g\left(Z_{i}\right)\right)\right| \\
& \leq \frac{2}{n} \sum_{i=1}^{n}\left|\kappa^{\top} \frac{\partial m\left(V_{i}, \alpha^{\top} X_{i}, g\left(Z_{i}\right)\right)}{\partial u}(\widehat{\alpha}-\alpha)^{\top} X_{i}\right|^{2} \\
& +\frac{2}{n} \sum_{i=1}^{n}\left|\kappa^{\top} \frac{\partial m\left(V_{i}, \alpha^{\top} X_{i}, g\left(Z_{i}\right)\right)}{\partial w}\left(\widehat{g}\left(Z_{i}\right)-g\left(Z_{i}\right)\right)\right|^{2} \\
& +\frac{2}{n} \sum_{i=1}^{n}\left|\kappa^{\top} \frac{\partial m\left(V_{i}, \alpha^{\top} X_{i}, g\left(Z_{i}\right)\right)}{\partial u}(\widehat{\alpha}-\alpha)^{\top} X_{i}\right|\left|\kappa^{\top} m\left(V_{i}, \alpha^{\top} X_{i}, g\left(Z_{i}\right)\right)\right| \\
& +\frac{2}{n} \sum_{i=1}^{n}\left|\kappa^{\top} \frac{\partial m\left(V_{i}, \alpha^{\top} X_{i}, g\left(Z_{i}\right)\right)}{\partial w}\left(\widehat{g}\left(Z_{i}\right)-g\left(Z_{i}\right)\right)\right|\left|\kappa^{\top} m\left(V_{i}, \alpha^{\top} X_{i}, g\left(Z_{i}\right)\right)\right| \\
& \leq\|\widehat{\alpha}-\alpha\|^{2} \frac{2}{n} \sum_{i=1}^{n}\left\|\frac{\partial m\left(V_{i}, \alpha^{\top} X_{i}, g\left(Z_{i}\right)\right)}{\partial u} \otimes X_{i}\right\|^{2} \\
& +\|\widehat{\beta}-\beta\|^{2} \frac{4}{n} \sum_{i=1}^{n}\left\|\frac{\partial m\left(V_{i}, \alpha^{\top} X_{i}, g\left(Z_{i}\right)\right)}{\partial w} \otimes \Phi_{k}\left(Z_{i}\right)\right\|^{2} \\
& +\frac{4}{n} \sum_{i=1}^{n}\left\|\frac{\partial m\left(V_{i}, \alpha^{\top} X_{i}, g\left(Z_{i}\right)\right)}{\partial w}\right\|^{2} \gamma_{k}^{2}\left(Z_{i}\right) \\
& +\|\widehat{\alpha}-\alpha\| \frac{2}{n}\left(\sum_{i=1}^{n}\left\|\frac{\partial m\left(V_{i}, \alpha^{\top} X_{i}, g\left(Z_{i}\right)\right)}{\partial u} \otimes X_{i}\right\|^{2}\right)^{1 / 2}\left(\sum_{i=1}^{n}\left\|m\left(V_{i}, \alpha^{\top} X_{i}, g\left(Z_{i}\right)\right)\right\|^{2}\right)^{1 / 2}
\end{aligned}
$$




$$
\begin{aligned}
& +\frac{2}{n}\left(\sum_{i=1}^{n}\left\|\frac{\partial m\left(V_{i}, \alpha^{\top} X_{i}, g\left(Z_{i}\right)\right)}{\partial w}\left(\widehat{g}\left(Z_{i}\right)-g\left(Z_{i}\right)\right)\right\|^{2}\right)^{1 / 2}\left(\sum_{i=1}^{n}\left\|m\left(V_{i}, \alpha^{\top} X_{i}, g\left(Z_{i}\right)\right)\right\|^{2}\right)^{1 / 2} \\
= & \|\widehat{\alpha}-\alpha\|^{2} O_{P}(q p)+\|\widehat{\beta}-\beta\|^{2} O_{P}(q k)+\sup _{z} \gamma_{k}^{2}(z) O_{P}(q)=o_{P}(1)
\end{aligned}
$$

by Assumptions A.1 and 4.2, where Cauchy-Schwarz inequality is used to show the last two sums are of smaller order. Thus, the assertion of (1) holds.

(2). We first consider

$$
\nu_{n}(\mathbf{a}, f ; \kappa)=\frac{1}{\sqrt{n}} \sum_{i=1}^{n} \kappa^{\top}\left(m\left(V_{i}, \mathbf{a}^{\top} X_{i}, f\left(Z_{i}\right)\right)-E\left[m\left(V_{i}, \mathbf{a}^{\top} X_{i}, f\left(Z_{i}\right)\right)\right]\right),
$$

for any $\kappa \in \mathbb{R}^{q}$ such that $\|\kappa\|=1$ and $(\mathbf{a}, f) \in \Theta$. Because of the convergence in Theorem 3.2 , we eventually will show $\nu_{n}(\widehat{\alpha}, \widehat{g} ; \kappa)-\nu_{n}(\alpha, g ; \kappa)=o_{P}(1)$.

Notice by the first order Taylor expansion that

$$
\begin{aligned}
& m\left(V_{i}, \mathbf{a}^{\top} X_{i}, f\left(Z_{i}\right)\right)-m\left(V_{i}, \alpha^{\top} X_{i}, g\left(Z_{i}\right)\right) \\
= & \frac{\partial m\left(V_{i}, \alpha^{\top} X_{i}, g\left(Z_{i}\right)\right)}{\partial u}(\mathbf{a}-\alpha)^{\top} X_{i}+\frac{\partial m\left(V_{i}, \alpha^{\top} X_{i}, g\left(Z_{i}\right)\right)}{\partial w}\left(f\left(Z_{i}\right)-g\left(Z_{i}\right)\right),
\end{aligned}
$$

for all $(\mathbf{a}, f)$ in the neighbourhood of $(\alpha, g)$, where $f$ has the form $\mathbf{b}^{\top} \Phi_{k}(\cdot)$. Thus,

$$
\begin{aligned}
& P\left(\sup _{\|(\mathbf{a}, f)-(\alpha, g)\|<\delta}\left|\nu_{n}(\mathbf{a}, f ; \kappa)-\nu_{n}(\alpha, g ; \kappa)\right|>\eta\right) \\
\leq & P\left(\sup _{\|(\mathbf{a}, f)-(\alpha, g)\|<\delta}\left|\frac{1}{\sqrt{n}} \sum_{i=1}^{n} \kappa^{\top}\left[\frac{\partial m}{\partial u}(\mathbf{a}-\alpha)^{\top} X_{i}-E \frac{\partial m}{\partial u}(\mathbf{a}-\alpha)^{\top} X_{i}\right]\right|>\eta / 2\right) \\
& +P\left(\sup _{\|(\mathbf{a}, f)-(\alpha, g)\|<\delta}\left|\frac{1}{\sqrt{n}} \sum_{i=1}^{n} \kappa^{\top}\left[\frac{\partial m}{\partial w}\left(f\left(Z_{i}\right)-g\left(Z_{i}\right)\right)-E \frac{\partial m}{\partial w}\left(f\left(Z_{i}\right)-g\left(Z_{i}\right)\right)\right]\right|>\eta / 2\right) \\
\leq & P\left(\sup _{\|(\mathbf{a}, f)-(\alpha, g)\|<\delta}\left|\frac{1}{\sqrt{n}} \sum_{i=1}^{n}\left[\kappa^{\top} \frac{\partial m}{\partial u} X_{i}-E \kappa^{\top} \frac{\partial m}{\partial u} X_{i}\right]^{\top}(\mathbf{a}-\alpha)\right|>\eta / 2\right) \\
& +P\left(\sup _{\|(\mathbf{a}, f)-(\alpha, g)\|<\delta}\left|\frac{1}{\sqrt{n}} \sum_{i=1}^{n}\left[\kappa^{\top} \frac{\partial m}{\partial w} \Phi_{k}\left(Z_{i}\right)-E \kappa^{\top} \frac{\partial m}{\partial w} \Phi_{k}\left(Z_{i}\right)\right]^{\top}(\mathbf{b}-\beta)\right|>\eta / 4\right) \\
& +P\left(\sup _{\|(\mathbf{a}, f)-(\alpha, g)\|<\delta}\left|\frac{1}{\sqrt{n}} \sum_{i=1}^{n}\left[\kappa^{\top} \frac{\partial m}{\partial w} \gamma_{k}\left(Z_{i}\right)-E \kappa^{\top} \frac{\partial m}{\partial w} \gamma_{k}\left(Z_{i}\right)\right]\right|>\eta / 4\right) \\
\leq & P\left(\sup _{\|(\mathbf{a}, f)-(\alpha, g)\|<\delta}\left\|\frac{1}{\sqrt{n p}} \sum_{i=1}^{n}\left[\kappa^{\top} \frac{\partial m}{\partial u} X_{i}-E \kappa^{\top} \frac{\partial m}{\partial u} X_{i}\right] \mid\right\| \sqrt{p}(\mathbf{a}-\alpha) \|>\eta / 2\right) \\
& +P\left(\sup _{\|(\mathbf{a}, f)-(\alpha, g)\|<\delta} \mid \frac{1}{\sqrt{n k}} \sum_{i=1}^{n}\left[\kappa^{\top} \frac{\partial m}{\partial w} \Phi_{k}\left(Z_{i}\right)-E \kappa^{\top} \frac{\partial m}{\partial w} \Phi_{k}\left(Z_{i}\right)\right]\|\| \sqrt{k}(\mathbf{b}-\beta) \|>\eta / 4\right) \\
& +P\left(\sup _{\|(\mathbf{a}, f)-(\alpha, g)\|<\delta}\left|\frac{1}{\sqrt{n}} \sum_{i=1}^{n}\left[\kappa^{\top} \frac{\partial m}{\partial w} \gamma_{k}\left(Z_{i}\right)-E \kappa^{\top} \frac{\partial m}{\partial w} \gamma_{k}\left(Z_{i}\right)\right]\right|>\eta / 4\right)
\end{aligned}
$$


$:=I_{1 n}+I_{2 n}+I_{3 n}, \quad$ say

Observe by the classical CLT that

$$
\begin{aligned}
\frac{1}{\sqrt{n p}} \sum_{i=1}^{n}\left[\kappa^{\top} \frac{\partial m}{\partial u} X_{i}-E \kappa^{\top} \frac{\partial m}{\partial u} X_{i}\right] & =O_{P}(1), \\
\frac{1}{\sqrt{n k}} \sum_{i=1}^{n}\left[\kappa^{\top} \frac{\partial m}{\partial w} \Phi_{k}\left(Z_{i}\right)-E \kappa^{\top} \frac{\partial m}{\partial w} \Phi_{k}\left(Z_{i}\right)\right] & =O_{P}(1) .
\end{aligned}
$$

It follows that if $\|\sqrt{p}(\mathbf{a}-\alpha)\|$ and $\|\sqrt{k}(\mathbf{b}-\beta)\|$ are sufficient small, $I_{1 n}<\varepsilon / 3$ and $I_{2 n}<\varepsilon / 3$. Meanwhile, using the condition that $\sqrt{n} \sup _{z}\left\|\gamma_{k}(z)\right\|=o(1)$ we have $I_{3 n}<\varepsilon / 3$. This shows that, in view of Theorem 3.2, when $n$ is large, $P\left(\left|\nu_{n}(\widehat{\alpha}, \widehat{g} ; \kappa)-\nu_{n}(\alpha, g ; \kappa)\right|>\eta\right)<\varepsilon$ for any given $\varepsilon, \eta>0$.

Furthermore, since

$$
\begin{gathered}
\frac{1}{\sqrt{n}} \sum_{i=1}^{n} \kappa^{\top}\left[m\left(V_{i}, \widehat{\alpha}^{\top} X_{i}, \widehat{\beta}^{\top} \Phi_{k}\left(Z_{i}\right)\right)-m\left(V_{i}, \alpha^{\top} X_{i}, g\left(Z_{i}\right)\right)\right] \\
=\nu_{n}(\widehat{\alpha}, \widehat{g} ; \kappa)-\nu_{n}(\alpha, g ; \kappa)+\sqrt{n} \bar{m}_{n}^{*}(\widehat{\alpha}, \widehat{g} ; \kappa)
\end{gathered}
$$

the assertion of (2) holds by virtue of Assumption 4.1. This finishes the proof.

Proof of Theorem 4.2. Because for any $(\mathbf{a}, \mathbf{b})$ and $\kappa$ with $\|\kappa\|=1$,

$$
\begin{aligned}
\frac{1}{\sqrt{n}} D_{n}(\mathbf{a}, \mathbf{b} ; \kappa) & =\left(E\left[\kappa^{\top} m\left(V_{1}, \mathbf{a}^{\top} X_{1}, \mathbf{b}^{\top} \Phi_{k}\left(Z_{1}\right)\right)\right]^{2}\right)^{1 / 2}+o_{P}(1) \\
& =\left(\kappa^{\top} E\left[m\left(V_{1}, \mathbf{a}^{\top} X_{1}, \mathbf{b}^{\top} \Phi_{k}\left(Z_{1}\right)\right) m\left(V_{1}, \mathbf{a}^{\top} X_{1}, \mathbf{b}^{\top} \Phi_{k}\left(Z_{1}\right)\right)^{\top}\right] \kappa\right)^{1 / 2}+o_{P}(1),
\end{aligned}
$$

which is bounded away from zero and infinity in probability, it suffices to show that there is some $\kappa^{*}$ with $\left\|\kappa^{*}\right\|=1$ such that

$$
\frac{1}{\sqrt{n}} \sum_{i=1}^{n} \kappa^{* \boldsymbol{\top}} m\left(V_{i}, \mathbf{a}^{\boldsymbol{\top}} X_{i}, \mathbf{b}^{\top} \Phi_{k}\left(Z_{i}\right)\right) \rightarrow_{P} \infty
$$

as $n \rightarrow \infty$ for any $(\mathbf{a}, \mathbf{b}) \in \mathbb{R}^{p+k}$.

Note by the Law of Large Number that

$$
\begin{aligned}
& \frac{1}{\sqrt{n}} \sum_{i=1}^{n} \kappa^{\top} m\left(V_{i}, \mathbf{a}^{\top} X_{i}, \mathbf{b}^{\top} \Phi_{k}\left(Z_{i}\right)\right)=\sqrt{n} \frac{1}{n} \sum_{i=1}^{n} \kappa^{\top} m\left(V_{i}, \mathbf{a}^{\top} X_{i}, \mathbf{b}^{\top} \Phi_{k}\left(Z_{i}\right)\right) \\
= & \sqrt{n}\left\{E\left[\kappa^{\top} m\left(V_{i}, \mathbf{a}^{\top} X_{i}, \mathbf{b}^{\top} \Phi_{k}\left(Z_{i}\right)\right)\right]+o_{P}(1)\right\} .
\end{aligned}
$$

Let $\kappa^{*}=E\left[m\left(V_{i}, \mathbf{a}^{\top} X_{i}, \mathbf{b}^{\top} \Phi_{k}\left(Z_{i}\right)\right)\right] /\left\|E\left[m\left(V_{i}, \mathbf{a}^{\top} X_{i}, \mathbf{b}^{\top} \Phi_{k}\left(Z_{i}\right)\right)\right]\right\|$. Then,

$$
\begin{aligned}
& \frac{1}{\sqrt{n}} \sum_{i=1}^{n} \kappa^{* \top} m\left(V_{i}, \mathbf{a}^{\top} X_{i}, \mathbf{b}^{\top} \Phi_{k}\left(Z_{i}\right)\right)=\sqrt{n}\left\{\left\|E\left[m\left(V_{i}, \mathbf{a}^{\top} X_{i}, \mathbf{b}^{\top} \Phi_{k}\left(Z_{i}\right)\right)\right]\right\|+o_{P}(1)\right\} \\
\geq & \sqrt{n}\left\{\inf _{(\mathbf{a}, h) \in \Theta}\left\|E\left[m\left(V_{i}, \mathbf{a}^{\top} X_{i}, h\left(Z_{i}\right)\right)\right]\right\|+o_{P}(1)\right\} \geq \sqrt{n}\left(\delta_{n}+o_{P}(1)\right) \rightarrow_{P} \infty,
\end{aligned}
$$

as $n \rightarrow \infty$, which finishes the proof. 


\section{References}

[1] Andrews, D. (1994). Asymptotics for semiparametric econometric models via stochastic equicontinuity. Econometrica, 62:43-72.

[2] Belloni, A., Chernozhukov, V., Chetverikov, D., and Kato, K. (2015). Some new asymptotic theory for least squares series: Pointwise and uniform results. Journal of Econometrics, 186:345-366.

[3] Belloni, A., Chernozhukov, V., and Wang, L. (2014). Pivitor estimation via square-root Lasso in nonparametric regression. Annals of Statistics, 42:757-788.

[4] Cattaneo, M. D., Jansson, M., and Newey, W. K. (2016). Alternative asymptotics and the partially linear model with many regressors. Econometric Theory, forthcoming:1-25.

[5] Cattaneo, M. D., Jansson, M., and Newey, W. K. (2017). Inference in linear regression models with many covariates and heteroscedasticity. Working paper.

[6] Chang, J., Chen, S., and Chen, X. (2015). High dimensional generalized empirical likelihood for moment restrictions with dependent data. Journal of Econometrics, 185:283-304.

[7] Chen, X. and Christensen, T. (2015). Optimal uniform convergence rates and asymptotic normality for series estimators under weak dependence and weak conditions. Journal of Econometrics, 188:447-465.

[8] Chen, X. and Liao, Z. (2015). Sieve semiparametric two-step GMM under weak dependence. Journal of Econometrics, 189:163-186.

[9] Chen, X., Linton, O., and Keilegom, I. V. (2003). Estimation for semiparametric models when the criterion function is not smooth. Econometrica, pages 1591-1608.

[10] Chen, X. and Pouzo, D. (2012). Estimation of nonparametric conditional moment models with possibly nonsmooth generalized residuals. Econometrica, 80:277-321.

[11] Dong, C. and Linton, O. (2016). Additive nonparametric models with time variable and both stationary and nonstationary regressors. https://papers.ssrn.com/sol3/papers.cfm? abstract id=2847681.

[12] Dudley, R. M. (2003). Real Analysis and Probability. Cambridge studies in advanced mathematics 74. Cambridge University Press, Cambridge, U.K.

[13] Engle, R., Granger, C. W. J., Rice, J., and Weiss, A. (1986). Semiparametric Estimates of the Relation Between Weather and Electricity Sales. Journal of the American Statistical Association, 81:310-320.

[14] Gao, J. and Liang, H. (1997). Statistical inference in single-index and partially nonlinear models. Annals of the Institute of Statistical Mathematics, 49(3):493-517.

[15] Gao, J. and Shi, P. (1997). M-type smoothing splines in non- and semi-parametric regression models. Statistica Sinica, 7(3):1155-1169.

[16] Gautschi, W. (2004). Orthogonal Polynomials: Computation and Approximation. Numerical Mathematics and Scientific Computation. Oxford University Press, Oxford. 
[17] Han, C. and Phillips, P. C. B. (2006). GMM with many moment conditions. Econometrica, 18(74):147192.

[18] Hansen, L. P. (1982). Large sample properties of generalized method of moments estimators. Econometrica, 50:1029-1054.

[19] Härdle, W., Liang, H., and Gao, J. (2000). Partially Linear Models. Springer-Verlag, New York.

[20] Mammen, E. (1989). Asymptotics with increasing dimension for robust regression with applications to the Bootstrap. Annals of Statistics, 17:382-400.

[21] Newey, W. K. (1997). Convergence rates and asymptotic normality for series estimators. Journal of Econometrics, 79:147-168.

[22] Newey, W. K. and Powell, J. L. (2003). Instrumental variable estimation of nonparametric models. Econometrica, 71:1565-1578.

[23] Newey, W. K. and Windmeijer, F. (2009). Generalized method of moments with many weak moment conditions. Econometrica, 77:687-719.

[24] Pakes, A. and Pollard, D. (1989). Simulation and the asymptotics of optimization estimators. Econometrica, 57:1027-1057.

[25] Portnoy, S. (1984). Asymptotic behaviour of M-estimators of $p$ regression parameters when $p^{2} / n$ is large. I: Consistency. Annals of Statistics, 12(4):1298-1309.

[26] Portnoy, S. (1985). Asymptotic behaviour of M-estimators of $p$ regression parameters when $p^{2} / n$ is large. II: Normal approximation. Annals of Statistics, 13(4):1403-1417.

[27] Powell, J. L. (1984). Estimation of Semiparametric Models. Handbook of Econometrics IV. Edited by R. F. Engle and D. L. McFadden. Elsevier, New York.

[28] Robinson, P. M. (1988). Root-N-consistent semiparametric regression. Econometrica, 56(2):931-954.

[29] Yatchew, A. (1997). An elementary estimator of the partial linear model. Economic Letters, 57(2):135143.

[30] Yatchew, A. (2003). Semiparametric Regression for the Applied Econometrician. Cambridge University Press, New York. 